TI 2018-033/III

Tinbergen Institute Discussion Paper
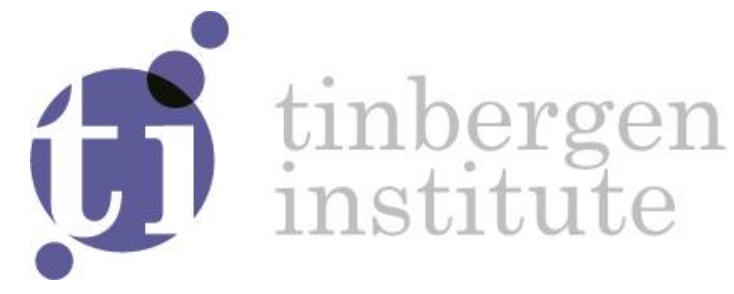

\title{
The option value of vacant land and the optimal timing of city extensions
}

Rutger-Jan Lange ${ }^{1}$

Coen Teulings ${ }^{2}$

${ }^{1}$ Erasmus University Rotterdam

2 University of Cambridge 
Tinbergen Institute is the graduate school and research institute in economics of Erasmus University Rotterdam, the University of Amsterdam and VU University Amsterdam.

Contact: discussionpapers@tinbergen.nl

More TI discussion papers can be downloaded at http://www.tinbergen.nl

Tinbergen Institute has two locations:

Tinbergen Institute Amsterdam

Gustav Mahlerplein 117

1082 MS Amsterdam

The Netherlands

Tel.: +31(0)205984580

Tinbergen Institute Rotterdam

Burg. Oudlaan 50

3062 PA Rotterdam

The Netherlands

Tel.: +31(0)10408 8900 


\title{
The option value of vacant land and the optimal timing of city extensions
}

\author{
Rutger-Jan Lange $^{1}$ and Coen N. Teulings ${ }^{2}$ \\ ${ }^{1}$ Econometric Institute, Erasmus University Rotterdam, Netherlands \\ ${ }^{2}$ Faculty of Economics, University of Cambridge, United Kingdom
}

\section{March 2018}

\begin{abstract}
Classic real options theory rests on two debatable assumptions: projects require a fixed investment and generate cash flows that follow a random walk. Relaxing both assumptions leads to radically different conclusions regarding the optimal timing of investment. We model investment using a Stone-Geary production function (Leontief and Cobb-Douglas are special cases) and growth as a mean-reverting Brownian motion. The solution method for this option valuation problem is non-trivial because the state space is two dimensional (level of the cash flow and its growth). For Leontief, the optimal policy is intuitive; the moment of investment involves a trade-off between the level of the cash flow and its growth. For Cobb-Douglas, in contrast, the optimal moment of investment depends only on the growth. More surprisingly, investment should be delayed when growth is high. This conclusion persists in the general Stone-Geary case. Applied to urban real estate, this suggests that up to $20 \%$ of cities should delay new construction because of high growth. The option value of vacant land may represent $60 \%$ of the value of new construction. High prices of vacant land may thus result from rational investor behavior rather than regulatory inefficiency. Our analysis should be widely applicable, for example to investment in high-growth companies.
\end{abstract}

keywords: real options, mean-reverting growth, real estate construction

JEL code: D81, E22, R11, R30 


\section{Introduction}

The standard model of real options rests on two assumptions: (i) the investment is fixed, and (ii) the potential cash flow evolves according to a random walk. These assumptions are convenient, since combined they allow an analytic solution to the optimal exercise strategy. However, both assumptions are questionable, especially for real estate. First, the growth of cities is highly persistent; Desmet and Rappaport (2015) show that the mean-reversion parameter in an AR(1) model of the population growth of American cities is around 0.95, while Nur and Teulings (in prep) report similar values for cities in the Netherlands. The mean-reversion parameter of log population is a good proxy for the mean-reversion of the rental value of housing in many models of cities. ${ }^{1}$ Second, as the density of construction varies widely between locations, the assumption of fixed investment is unlikely to hold. This paper shows that relaxing both assumptions simultaneously has major implications for the optimal timing and density of construction.

First, consider the assumption of the cash flow being a random walk. This implies that current growth has no predictive power for future growth. If this were true, the price of vacant land in the proximity of a city - i.e. the option value of future construction would be independent of its current growth rate, which is unrealistic. One can make a similar argument for firms. If current growth has no predictive power for future growth, then what would explain high multiples for IT companies like Google and Facebook compared to firms in more traditional industries, like Procter \& Gamble and Shell? Indeed, Chan et al. (2003) find persistence in sales revenue growth. ${ }^{2}$ One could assume that the sales growth of such companies in the recent past reflects a higher drift, but this assumption has undesirable implications of its own. The mean growth of some IT firms over the past decade has exceeded any reasonable discount rate. If this excess growth were permanent, then the value of such firms would be unbounded. Growth differentials between cities - and firms, for that matter - are thus likely to be persistent but not permanent. We therefore model the growth of the potential cash flow as a mean-reverting process, or in continuous time, as an Ornstein-Uhlenbeck process. The complication of this assumption is that the state space becomes two dimensional: consisting of both the level of the cash flow and its growth.

Second, consider the assumption that exercising the option requires an investment of fixed size. This implies that the investor must decide only on the optimal timing of investment, not on its size. If this were true, the density of construction would be equal across different plots of land. Making the size of the investment endogenous is particularly relevant when this investment is irreversible in both upward and downward

\footnotetext{
${ }^{1}$ See Section 6 for a detailed discussion.

${ }^{2}$ They also find that persistent sales growth does not necessarily translate into persistent earnings growth, which may be due to the "noisiness of the earnings yield measure" (p. 683). However, they do not estimate an $\mathrm{AR}(1)$ model, making a direct comparison with our method hard.
} 
directions. For real estate, irreversibility is evidently true for downsizing (i.e. scrapping the building; see Glaeser and Gyourko (2005)). However, extending an existing building is also far more costly than greenfield construction of new buildings. ${ }^{3}$ For these reasons, this paper assumes that construction on a given plot of land is irreversible in both directions. We assume that housing services are produced by a Stone-Geary technology with land and construction as its inputs. This production function has two limiting special cases: (i) Leontief, where the size of the investment in construction is fixed so that the elasticity of substitution between land and construction is zero (as in the standard real options case), and (ii) Cobb-Douglas, where there is no fixed investment and the elasticity of substitution is equal to unity. In the general Stone-Geary case, the elasticity of substitution between land and construction falls between zero and one.

The combination of both generalizations of the standard problem leads to counterintuitive results. Consider introducing persistence in the growth of the cash flow while maintaining the assumption of a Leontief technology. Then, one would intuitively expect the optimal stopping boundary to reflect a trade-off between the level of the cash flow and its growth. The investor invests either because the current cash flow is high (even though growth is low) or because growth is high (even though the cash flow is low). We show that this intuition holds true. However, this naive expectation breaks down in the other limiting case. For Cobb-Douglas, we find two surprising results. First, the optimal exercise policy depends exclusively on the growth of the cash flow and not its level. The intuition is that optimal investment in construction is always a fixed share of the value of the asset after construction, as is standard for a Cobb-Douglas production function with an elasticity of substitution between land and construction equal to unity. Second, even more surprisingly, there is a maximum growth rate above which investment should be delayed. That is, when the prospects of the project are particularly bright, the investor should postpone investment! There are two reasons for this counterintuitive result. Delaying investment allows new information on the future growth of the cash flow to come to light, which can improve decision making on the optimal density of construction, with investors choosing a higher density when the new information is favorable. This explains why the combination of persistence in growth and variable investment matters. Further, the high growth rate implies that the optimal density of construction is higher than is justified by the current level of the cash flow. The current cash flow may not even cover the interest bill on the investment, in which case postponing investment is clearly optimal.

The counterintuitive finding that construction should be delayed for high growth rates generalizes to the Stone-Geary case. For Stone-Geary, the optimal investment boundary is U-shaped as a function of growth: investment should be delayed when the growth

\footnotetext{
${ }^{3}$ One might argue that demolition and rebuilding is a common practice, in particular in US cities. However, just an eyeball test shows that there is a strong persistence in urban structures. Given an $\mathrm{AR}(1)$ parameter of 0.95 in the growth rate, a persistence of 20 years would justify our analysis.
} 
rate is very low (as in the Leontief case) or very high (as in the Cobb-Douglas case). Our numerical results show that this phenomenon is important for empirically relevant parameters. Even for low values of the marginal construction-share (e.g. 10\%; $0 \%$ yields the Leontief case), the curve is upward sloping for empirically relevant growth rates. Indeed, for reasonable parameter values, up to $20 \%$ of cities should delay investment due to high growth. Naturally, the optimal density of construction along the optimal stopping boundary increases as the growth rate increases. However, the option value of vacant land on the edge of high-growth cities also increases as a function of the growth rate; indeed, it may constitute up to $60 \%$ of the value of newly constructed houses. High-growth cities should therefore wait longer before transforming vacant land into built area, even though the value of the land is much higher than is justified by agricultural use. This conclusion has important policy implications. Glaeser and Gyourko (2002) argue that the high land prices in the proximity of growing cities is evidence of inefficient planning restrictions. Our analysis shows that this conclusion cannot be based on the price of the vacant land alone.

The practical relevance of this question would suggest that it must have been answered long ago, but this turns out not to be the case. Dixit and Pindyck (1994) discuss the option value when the level of the cash flow is mean-reverting. However, mean-reversion in the level has entirely different implications than mean-reversion in growth. Arnott and Lewis (1979) and Capozza and Helsley (1989) apply a model with permanent growth rate differentials between cities to show that land close to fast-growing cities commands a higher option value and that this land will be developed with a higher density of construction. The model that comes closest to ours is that of Capozza and Li (1994), which allows for stochastic non-persistent growth around a deterministic trend that differs between cities. ${ }^{4}$ However, as discussed above, though this model has the merit of convenience, it does not capture reality very well. By contrast, in our model growth rate differentials are persistent, but not permanent. Under this more realistic assumption, unexpected shocks to the growth rate have a much larger effect on land prices, since a growth shock affects not only today's growth rate, but also tomorrow's. Persistence in growth might therefore contribute to our understanding of the excess volatility in asset prices relative to the volatility in the underlying cash flows. We expect therefore that our analysis of the valuation of asset prices for cash flows with an Ornstein-Uhlenbeck growth process has a wider application than real estate; for example, in the valuation of equity of high-growth

\footnotetext{
${ }^{4}$ See their discussion on the comparative statics of growth rate $g$ on pp. 896-897. They suggest using of a model with stochastic rather than deterministic growth rates, as we do in this paper. Interestingly, Capozza and Li (1994) conjecture our results:
}

We conjecture that, in a more sophisticated rational-expectations model with stochastic rather than constant growth expectations, this phenomenon (...) will imply that additional projects will be undertaken even as growth expectations are declining. 
companies.

The analysis is, from a technical point of view, rather involved. This is because (i) the problem has two state variables: the level of the cash flow and its growth; (ii) the option is of the American type, meaning that the decision-maker can exercise the option at any time; and (iii) the stochastic process is parabolic rather than elliptic ${ }^{5}$, implying that classic tools such as 'smooth fit' are not available to us. In fact, this paper seems to be the first to document the failure of smooth fit in higher dimensions. Even in the elliptic case, multidimensional American-type option valuation problems are notoriously difficult to solve. Prominent contributions to the growing body of literature in this area are Rogers (2002), Andersen and Broadie (2004) and Bally and Printems (2005). We deviate from the literature in permitting our investor to invest at random moments in time that arrive at a fixed Poisson arrival rate $\lambda$. The standard case, where investors may invest at any point in time, is obtained in the limit $\lambda \rightarrow \infty$. This problem set-up has rarely been considered in the literature; e.g. Rogers and Zane (2002) use the case $\lambda<\infty$ to model liquidity constraints. Apart from its theoretical appeal, our generalization is useful from a practical point of view, since the problem corresponding to $\lambda$ large but finite turns out to be mathematically and numerically more tractable than the problem corresponding to $\lambda=\infty$. In particular, we present a new method that finds the solution as a monotone (non-decreasing) sequence of lower bounds; moreover, this property persists through the discretization. These desirable properties are related to those observed in the literature on the 'penalty method'; see e.g. Zvan et al. (1998); Forsyth and Vetzal (2002); d'Halluin et al. (2004), and Zhang et al. (2008, 2009). As our numerical method is stable and straightforward to implement, we expect it to have wider applicability in the analysis of multidimensional American-type options.

This paper is structured as follows. Section 2 sets out the assumptions of the model for the Leontief case. Section 3 derives the present value of a cash flow with mean-reverting growth, while Section 4 presents the solution to the option valuation problem. Section 5 analyses the generalization to the Stone-Geary case, and Section 6 fleshes out our framework to the spatial structure of a city. Finally, Section 7 discusses the implications of our findings and contains suggestions for future work.

While the arguments in this paper are economically motivated, some level of technical detail is unavoidable. Readers mainly interested in our economic rationale may wish to skip the more technical subsections, the subheadings of which are preceded by an asterisk.

\footnotetext{
${ }^{5}$ This is because the $2 \times 2$ diffusion matrix of the process is singular; such processes are often called degenerate.
} 


\section{The model for the Leontief case}

\subsection{Assumptions}

We consider the value of an option to build an asset that yields a cash flow determined by a two-dimensional stochastic diffusion process $\left\{X_{t}\right\}_{t \geq 0}$ in continuous time defined on some complete filtered probability space $(\Omega, \mathcal{F}, \mathbb{P})$ satisfying the usual conditions. Specifically, we assume that the diffusion consists of two components, i.e. $X_{t}=\left(g_{t}, \ln f_{t}\right)^{\prime}$, where the first component $g_{t}$ is an Ornstein-Uhlenbeck process as in Uhlenbeck and Ornstein (1930) and where the second component $\ln f_{t}$ is defined as the integral of the first (i.e. $\ln f_{t}=\ln f_{0}+\int_{0}^{t} g_{\tau} d \tau ; f_{t}$ is the cash flow and $g_{t}$ is the (geometric) growth rate of the cash flow, both at time $t)$. In other words, we assume that $X_{t}=\left(g_{t}, \ln f_{t}\right)^{\prime}$ is the strong unique solution to the following pair of stochastic differential equations (SDEs):

$$
\begin{aligned}
\mathrm{d} g_{t} & =-\theta\left(g_{t}-\bar{\mu}\right) \mathrm{d} t+\bar{\sigma} \mathrm{d} W_{t}, \\
\mathrm{~d} \ln f_{t} & =g_{t} \mathrm{~d} t,
\end{aligned}
$$

where $W_{t}$ is a standard Wiener process. ${ }^{6}$ We note that $\ln f_{t}$ is perfectly predictable over time scales of order $\mathrm{d} t .^{7}$ The first stochastic process is the persistent, but mean-reverting, geometric growth rate of the second process. Specifically, $\bar{\mu}$ is the long-run mean of the growth rate, while $\theta>0$ is the rate of convergence to this long-run mean and $\bar{\sigma}>0$ is the standard deviation of innovations in the growth rate. Future payoffs are discounted at a rate $\bar{\rho}$ and we assume that the realization of the asset requires an irreversible investment $\mathcal{I}$. For the discussion of our numerical results, it useful to have a set of benchmark parameters. Nur and Teulings (in prep) estimate the Ornstein-Uhlenbeck mean-reversion parameter on an annual time scale to be $\theta \approx 0.05$, using population data for a sample of Dutch agglomerations. ${ }^{8}$ We use an interest rate $\bar{\rho}=0.04$ and a drift $\bar{\mu}=0.01$. The value of $\bar{\sigma}^{2}$ can most easily be assessed starting from the steady-state variance of population growth rate across cities, $\operatorname{Var}\left[g_{t}\right]$. We take as a point of reference a standard deviation of the growth rate across cities in the steady state of about 0.02 , implying $\operatorname{Var}\left[g_{t}\right]=4 \times 10^{-4}$. The steady-state distribution of the growth rate is $g_{t} \sim N\left[\bar{\mu}, \bar{\sigma}^{2} /(2 \theta)\right]$. Hence, $\bar{\sigma}^{2}=2 \theta \times \operatorname{Var}\left[g_{t}\right]=4 \times 10^{-5}$. This motivates the following definition:

Definition 1 Benchmark parameters on an annual time scale:

$$
\theta=0.05, \quad \bar{\rho}=0.04, \quad \bar{\mu}=0.01, \quad \bar{\sigma}^{2}=4 \times 10^{-5} .
$$

\footnotetext{
${ }^{6} \mathrm{~A}$ standard Wiener process satisfies $\mathrm{E}\left[W_{t}-W_{0}\right]=0, \mathrm{E}\left[W_{t}-W_{0}\right]^{2}=t$.

${ }^{7}$ This implies the $2 \times 2$ diffusion matrix of the process $\left\{X_{t}\right\}_{t \geq 0}$ is singular. Such processes are often called degenerate.

${ }^{8}$ Note that the mean-reversion parameter in a (discrete-time) AR(1) process is different from that in a (continuous-time) Ornstein-Uhlenbeck process.
} 


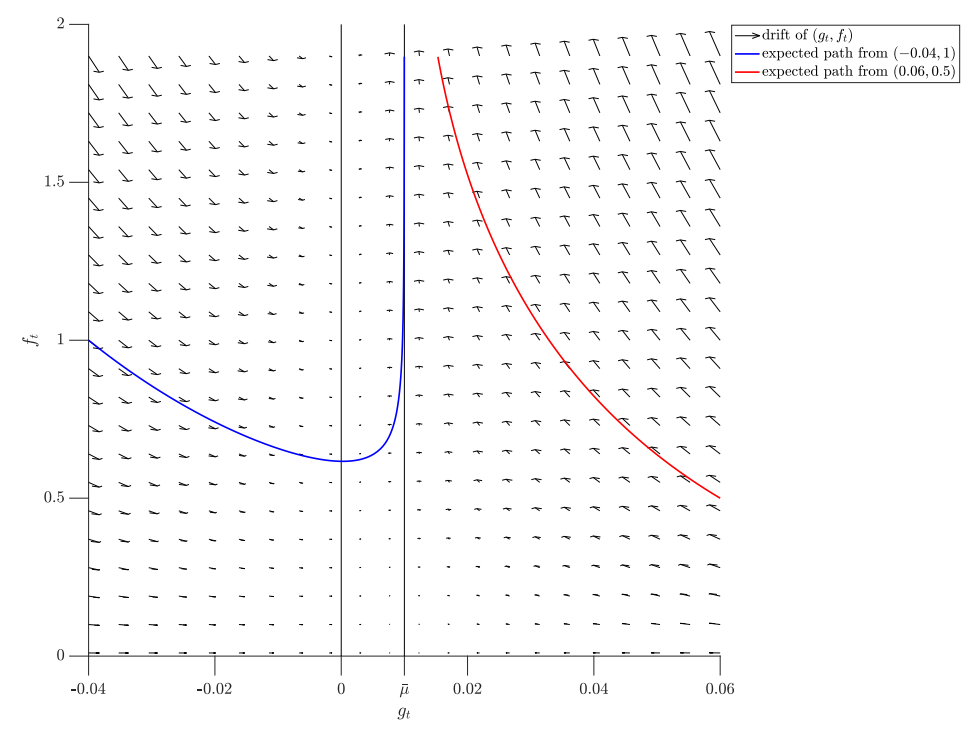

Figure 1: A flowchart of the bivariate stochastic process $\left(g_{t}, f_{t}\right)^{\prime}$ for the benchmark parameters.

Figure 1 provides a flowchart of the system in $\left(g_{t}, f_{t}\right)$ space. It displays two paths constructed by following the drift of the process and ignoring any noise, starting from $\left(g_{t}, f_{t}\right)=(-0.04,1)$ and $\left(g_{t}, f_{t}\right)=(0.06,0.5)$, respectively. The growth rate $g_{t}$ meanreverts to the drift parameter $\bar{\mu}$. Hence, both paths converge to the vertical line $g_{t}=\bar{\mu}$. The path starting from $(-0.04,1)$ declines until the growth rate passes the line $g_{t}=0$ and increases afterwards.

The model has four special cases related to the limits $\bar{\sigma}^{2}=0, \theta=0, \theta=\infty$, and both $\bar{\sigma}^{2}=\theta=0$.

- Deterministic growth: in the limit $\bar{\sigma}^{2} \rightarrow 0$ there is no uncertainty. The growth rate $g_{t}$ mean-reverts deterministically along an exponential path back to its mean $\bar{\mu}$ at a deterministic rate $\theta$. The long-term behavior is determined by $\bar{\mu}$.

- Immediate mean-reversion: the limit $\theta \rightarrow \infty$ is equivalent to $\bar{\sigma}^{2} \rightarrow 0$ (i.e.: deterministic growth). The growth rate $g_{t}$ mean-reverts immediately to $\bar{\mu}$, and stays there for eternity. The value of $\bar{\sigma}^{2}$ becomes irrelevant, since innovations are immediately suppressed. The growth is fully determined by $\bar{\mu}$.

- No mean-reversion: in the limit $\theta \rightarrow 0$, the growth rate $g_{t}$ follows a random walk without mean-reversion to $\bar{\mu}$. Hence, the value of $\bar{\mu}$ becomes irrelevant. In this case the asset value of the project is unbounded, as will be shown below.

- Degenerate case: when both $\bar{\sigma}^{2}$ and $\theta$ are zero, there is neither randomness nor mean-reversion. Hence, $g_{t}$ stays forever constant at its present value $g_{0}$. 
It is convenient to rewrite this process applying a transformed time scale $s$, such that the mean-reversion parameter is equal to unity. Let $s$ be the transformed time scale (hence, $s=\theta t$ ) and let $x_{s}, \rho, \mu, \sigma^{2}$ and $\psi$ be the growth rate and the parameters, respectively, that correspond to this transformation of the time scale. They are defined as

$$
\begin{aligned}
\rho & :=\frac{\bar{\rho}}{\theta}, \quad \mu:=\frac{\bar{\mu}}{\theta}, \quad \sigma^{2}:=\frac{\bar{\sigma}^{2}}{\theta^{3}}, \quad \psi:=\rho-\mu-\frac{1}{2} \sigma^{2}, \\
x_{s} & :=\frac{g_{t}}{\theta}-\mu-\sigma^{2},
\end{aligned}
$$

where $\psi$ is the modified discount rate, accounting for the expected future growth of the cash flow and for the randomness in future growth. The transformed growth rate $x_{t}$ is modified along two dimensions: (i) it is adjusted to the transformed time scale $s$ and (ii) it is measured in deviation from the expected long-term growth of the cash flow, see Proposition 1 below. We use equation (2.2) to transform the benchmark parameters to the new time scale.

Definition 2 The transformed benchmark parameters are

$$
\rho=0.80, \quad \mu=0.20, \quad \sigma^{2}=0.32, \quad \psi=0.44
$$

All figures use these benchmark parameters unless stated otherwise. Recall that an investment $\mathcal{I}$ is required for the realization of the asset. The flow of interest payments on an investment $\mathcal{I}$ per unit of the transformed time scale $s$ is equal to $\rho \mathcal{I}$. Let $y$ denote the transformed cash flow. It is convenient to rescale the project such that the transformed cash flow is equal to the interest payment on the investment $\mathcal{I}$ for $y=1$. Hence,

$$
y:=\frac{f}{\theta \rho \mathcal{I}}=\frac{f}{\bar{\rho} \mathcal{I}}
$$

while the investment required for the realization of this asset is equal to $\rho^{-1}$. Using these definitions, the stochastic differential equations corresponding to equation (2.1) read

$$
\begin{aligned}
\mathrm{d} x_{s} & =-\left(x_{s}+\sigma^{2}\right) \mathrm{d} s+\sigma \mathrm{d} W_{s}, \\
\mathrm{~d} \ln y_{s} & =\left(x_{s}+\mu+\sigma^{2}\right) \mathrm{d} s .
\end{aligned}
$$

Henceforth, we work in the transformed time scale $t \rightarrow s$ and the transformed state variables $(g, f) \rightarrow(x, y)$.

We deviate from the literature in assuming that the set of permissible investment times $\mathcal{T}_{\lambda}$ set is generated independently of the diffusion $\left\{X_{t}\right\}_{t \geq 0}$ by a Poisson process with arrival rate $\lambda \in(0, \infty)$. The decision-maker is permitted to invest at one of the Poisson arrival times; between two Poisson arrival times she is forced to wait. In our problem formulation, therefore, the set $\mathcal{T}_{\lambda}$ consists of all Poisson arrival times $\left\{\tau_{1}, \tau_{2}, \tau_{3}, \ldots\right\}$. As 
the intensity $\lambda$ of our Poisson process increases to infinity, opportunities to invest are generated continuously. This implies that classical stopping problems in the literature are a limiting case of our (more general) formulation. Apart from its theoretical appeal, our generalization is useful from a practical point of view, since the problem corresponding to $\lambda$ large but finite turns out to be mathematically and numerically more tractable than the problem corresponding to $\lambda=\infty$, as will be shown in Section 5 .

\section{$2.2{ }^{*}$ The distribution of $(x, y)$}

This subsection derives the joint distribution of $(x, y)$. Subsections preceded by an asterisk may be skipped by readers mainly interested in our economic rationale.

Proposition 1 The distribution of the vector-valued stochastic process $\left(x_{s}, \ln y_{s}\right)^{\prime}$ conditional on $\left(x_{0}, \ln y_{0}\right)^{\prime}$ is multivariate normal with a mean $m_{s}$ and covariance matrix $\Sigma_{s}$, where $m_{s}$ and $\Sigma_{s}$ are given by

$$
\begin{aligned}
& m_{s}=\left(\begin{array}{c}
-\sigma^{2}+\left(x_{0}+\sigma^{2}\right) \mathrm{e}^{-s} \\
\ln y_{0}+\mu s+\left(x_{0}+\sigma^{2}\right)\left(1-\mathrm{e}^{-s}\right)
\end{array}\right) \\
& \Sigma_{s}=\frac{1}{2} \sigma^{2}\left(\begin{array}{cc}
1-\mathrm{e}^{-2 s} & \left(1-\mathrm{e}^{-s}\right)^{2} \\
\left(1-\mathrm{e}^{-s}\right)^{2} & 2 s-4\left(1-\mathrm{e}^{-s}\right)+\left(1-\mathrm{e}^{-2 s}\right)
\end{array}\right) .
\end{aligned}
$$

In the steady state, we have

$$
\begin{aligned}
x_{s} & \sim \mathrm{N}\left(-\sigma^{2}, \frac{1}{2} \sigma^{2}\right), \\
\lim _{s \rightarrow \infty} \frac{\ln y_{s}-\ln y_{0}}{s} & \sim \mathrm{N}\left(\mu, \sigma^{2}\right) .
\end{aligned}
$$

Proofs of all propositions are presented in the Appendix. To the best of our knowledge, this paper provides the first representation of the joint distribution of an Ornstein-Uhlenbeck process and its integral. It follows from Proposition 1 that the marginal distributions of $x_{s}$ and $\ln y_{s}$ are again normal, with means and variances obtained by dropping irrelevant rows and columns from $m_{s}$ and $\Sigma_{s}$. In the steady state, $\mathrm{E} x=-\sigma^{2}=-0.32$, while the standard deviation of $x$ is $\sigma / \sqrt{2}=0.40$ for our benchmark parameters (see Definition 2).

Remark 1 The correlation between a zero-drift Brownian motion and its integral is $\sqrt{3} / 2 \approx 86 \%$. This follows from Proposition 1 , which by $e^{-s}=1-s+O\left(s^{2}\right)$ implies that the joint distribution of $\left(x_{s}, \ln y_{s}\right)^{\prime}$ for small $s$ (or, equivalently, $\theta=0$ ) is normal with mean $m_{s}=\left[x_{0}, \ln y_{0}+\left(x_{0}+\mu+\sigma^{2}\right) s\right]^{\prime}$ and covariance matrix $\Sigma_{s}=\sigma^{2}\left[\left(\frac{1}{3} s^{3}, \frac{1}{2} s^{2}\right),\left(\frac{1}{2} s^{2}, s\right)\right]$. 


\section{The asset value}

The asset value of the project described in section 2 as a function of the state variables $x_{s}$ and $y_{s}$ at time $s$, denoted $A\left(x_{s}, y_{s}\right)$, is the present value of all expected future cash flows

$$
A\left(x_{s}, y_{s}\right)=\mathrm{E}_{s}\left[\int_{s}^{\infty} y_{t} \mathrm{e}^{-\rho(t-s)} \mathrm{d} t\right]
$$

where $\mathrm{E}_{s}$ represents an expectation conditional on the state variables $\left(x_{s}, y_{s}\right)$. The standard method to characterize this asset value is through the Bellman equation corresponding to the dynamic process (2.5). In our case, this equation takes the form

$$
\begin{aligned}
y & :=(\rho-\mathcal{L}) A(x, y), \quad \forall(x, y) \in \mathbb{R} \times \mathbb{R}_{\geq 0}, \quad \text { where } \\
\mathcal{L} & :=\left(x+\mu+\sigma^{2}\right) y \mathrm{~d} / \mathrm{d} y-\left(x+\sigma^{2}\right) \mathrm{d} / \mathrm{d} x+\frac{1}{2} \sigma^{2} \mathrm{~d}^{2} / \mathrm{d} x^{2},
\end{aligned}
$$

where $\mathbb{R}_{\geq 0}$ denotes the set of non-negative real numbers, while $\mathcal{L}$ is a parabolic ${ }^{9}$ differential operator of first and second order in $x$ and $y$, respectively. This operator is known as the 'generator' of the process; see Karatzas and Shreve (2012). The return $\rho-\mathcal{L}$ on the asset value $A(x, y)$ is equal to the cash flow. The first term of the operator $\mathcal{L}$ captures the effect on the asset value of the change in $y$ over the interval $\mathrm{d} s$, which is equal to the growth rate $x+\mu+\sigma^{2}$ times the level of the cash flow $y$. The second term captures the effect of the mean-reversion of the growth rate back to its expected level $-\sigma^{2}$. The third term follows from Itô's lemma and captures the effect of the randomness of $x$. If $A(x, y)$ were linear in $x$, this effect would be zero since $\mathrm{E}_{s}[\mathrm{~d} x]=0$. However, because $A(x, y)$ is non-linear and $\mathrm{E}\left[(\mathrm{d} x)^{2} / \mathrm{d} s\right]=\sigma^{2}$, this effect cannot be ignored. The operator $\mathcal{L}$ contains no second derivative with respect to $y_{0}$, because the evolution of $y_{t}$ is non-random over time scales of order $\mathrm{d} s, \mathrm{E}\left[(\mathrm{d} y)^{2} / \mathrm{d} s\right]=0$.

This Bellman equation has no analytic solution, but we can use the joint distribution of $x_{s}$ and $\ln y_{s}$ conditional on the current state $\left(x_{0}, \ln y_{0}\right)$ given in Proposition 1 . Since the diffusion $\left\{X_{s}\right\}_{s \geq 0}$ is Markov, we can focus on the calculation of the asset value at time 0 . As $\ln y_{s}$ conditional on $\ln y_{0}$ is normal, it follows that $y_{s}$ is log-normal. The expectation of a $\log$-normal variable follows from $\operatorname{E} \exp \mathrm{N}\left(a, b^{2}\right)=\exp \left(a+\frac{1}{2} b^{2}\right)$. Therefore, the asset value given in equation (3.1) can be written as

$$
\begin{aligned}
A(x, y) & =\int_{0}^{\infty} \exp \left[m_{2, s}+\frac{1}{2} \Sigma_{22, s}-\rho s\right] \mathrm{d} s=: \text { y } a(x), \\
a(x) & :=\int_{0}^{\infty} \exp \left[-\psi s+x\left(1-e^{-s}\right)+\frac{\sigma^{2}}{4}\left(1-e^{-2 s}\right)\right] \mathrm{d} s
\end{aligned}
$$

\footnotetext{
${ }^{9}$ The absence of a second derivative with respect to $y$ implies that the differential operator $\mathcal{L}$ is parabolic rather than elliptic. This implies that many classic tools for option valuation, such as the principle of 'smooth fit', can no longer be relied upon. Such degenerate cases have not been considered in the literature; even classic texts such as Øksendal (2007), Peskir and Shiryaev (2006), and Karatzas and Shreve (2012) treat only the elliptic case.
} 
where $m_{2, s}$ and $\Sigma_{22, s}$ denote relevant entries of the vector $m_{s}$ and matrix $\Sigma_{s}$ in Proposition 1. The expression for $a(x)$ in the second line follows from substituting these entries in the first equality in the first line. Note that the state variable $y$ factors out: an increase in $y$ affects the asset value $A(x, y)$ proportionally. The fact that $A(x, y)$ can be multiplicatively decomposed into the effects of $y$ and $a(x)$ facilitates our analysis. For the integral in the second line to converge - and hence for the asset value to exist - we need:

$$
\psi>0 \quad \Longleftrightarrow \quad 0 \leq \sigma^{2}<2(\rho-\mu) .
$$

In the sequel, we assume this condition to be satisfied, as is the case for our benchmark parameters. It appears that our solution (3.3) is new. While this may be somewhat surprising, this method of expressing the asset value is useful given that the standard Bellman equation (3.2) has no analytic solution. Our solution $A(x, y)$ in equation (3.3) satisfies the PDE in equation (3.2), as can be verified by differentiating under the integral sign.

\section{$3.1 \quad *$ Numerical characterization of $a(x)$}

Although a closed-form solution for the integral for $a(x)$ in equation (3.3) does not exist, we can derive some useful properties of this function. Moreover, we and provide a simple and robust approximation.

Proposition 2 For $\forall x \in \mathbb{R}$, a $(x)$ satisfies

$$
\begin{array}{ccc}
1 & : & a(0)=\frac{1}{2} \exp \left(\frac{\sigma^{2}}{4}\right)\left(\frac{\sigma}{4}\right)^{-\psi / 2} \gamma\left(\frac{\psi}{2}, \frac{\sigma^{2}}{4}\right), \\
2: & 0<a^{\prime \prime}(x)<a^{\prime}(x)<a(x), \\
3: & a^{\prime \prime}(x) a(x)>a^{\prime}(x)^{2}, \\
4: & \lim _{x \rightarrow-\infty}\left(\psi-\frac{\sigma^{2}}{2}-x\right) a(x)=1, \\
5 & : & \lim _{x \rightarrow \infty}\left[x^{\psi} \exp \left(-x-\frac{\sigma^{2}}{4}\right) a(x)\right]=\Gamma(\psi),
\end{array}
$$

where $\Gamma(\cdot)$ is the Gamma function and $\gamma(\cdot)$ the incomplete Gamma function; see Appendix A.2.

Proposition 2 implies that $\lim _{x \rightarrow-\infty} a^{\prime}(x) / a(x)=0, \lim _{x \rightarrow-\infty} a(x)=0$, and $\lim _{x \rightarrow \infty} a^{\prime}(x) / a(x)=$ 1. For the special case of deterministic growth, $\left(\sigma^{2}=0\right)$ we can provide an analytic solution, since the third factor in the integrand in equation (3.3) vanishes and the remaining terms can be solved:

$$
\left.a(x)\right|_{\sigma^{2}=0}=e^{x} x^{-\psi} \gamma(\psi, x),\left.\quad a(0)\right|_{\sigma^{2}=0}=\psi^{-1} .
$$

This expression is also useful when $\sigma^{2}>0$, since it allows us to formulate lower and upper bounds for $a(x)$. Because $1-e^{-2 s} \in[0,1)$ for $s \in \mathbb{R}_{\geq 0}$, the contribution of the third factor 


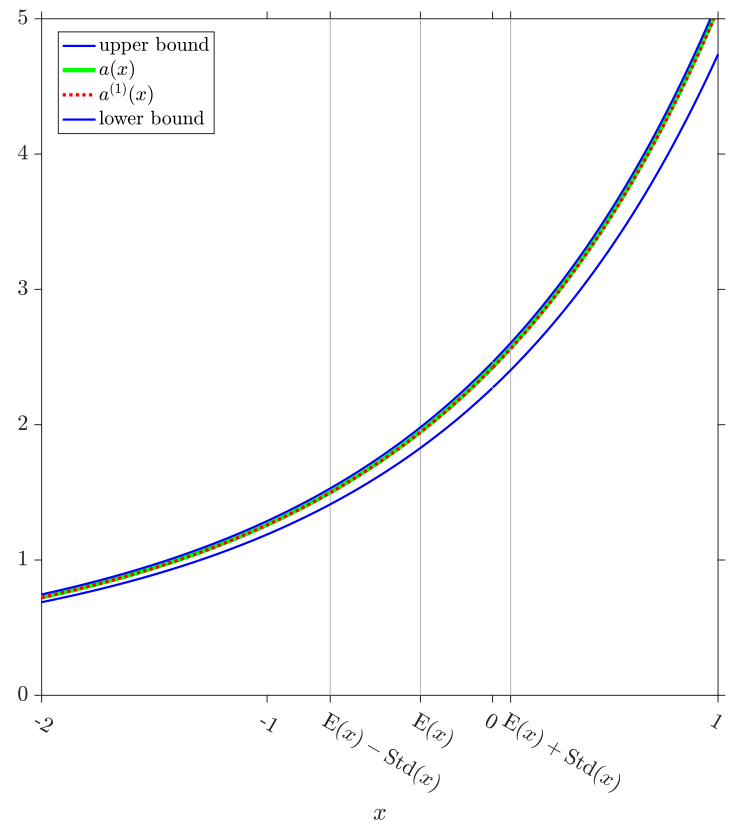

Figure 2: The functions $a(x), a(x)^{\text {(lower bound) }}, a(x)^{\text {(upper bound) }}$, and $a^{(1)}(x)$ defined in Remark 2.

in the integrand in equation (3.3) is positive for all $s$. A lower bound for $a(x)$ can thus be obtained by ignoring this term, while an upper bound can be obtained by setting it equal to its maximum value of $\sigma^{2} / 4$. Hence,

$$
\begin{aligned}
a(x)^{(\text {lower bound })} & =e^{x} x^{-\psi} \gamma(\psi, x) \\
a(x)^{(\text {upper bound })} & =\exp \left(\frac{\sigma^{2}}{4}\right) a(x)^{(\text {lower bound })}
\end{aligned}
$$

Remark 2 A convenient and accurate approximation of a $(x)$ can be obtained by a Taylor series approximation of a $(x)$ starting from the lower bound $a(x)^{(\text {lower bound) }}$ in equation (3.5) which is accurate even for low orders of the approximation. We can prove that

$$
a^{(n)}(x)<a^{(n+1)}(x)<a(x)<a(x)^{(\text {upper bound) }}, \forall n \geq 0, \forall x \in \mathbb{R}
$$

where the superscript $(n)$ denotes the order of the Taylor approximation. In practice, the upper bound gives a very accurate approximation of a $(x)$ for almost all parameter values. This is illustrated in Figure 2: $a^{(n)}(x)$ is very close to a $(x)^{\text {(upper bound) }}$, even for $n=1$. Since $a^{(1)}(x)<a(x)<a(x)^{\text {(upper bound) }}, a(x)^{\text {(upper bound) }}$ provides an accurate approximation of a $(x)$ for most practical purposes. 


\subsection{Excess short run volatility of the asset value}

Proposition 3 compares the short and long run volatility of the cash flow and the asset value in response to a shock in the growth rate $x_{0}$.

\section{Proposition 3}

1. The infinitesimal drift and variance of $\ln A(x, y)$ satisfy

$$
\begin{aligned}
\mathrm{E}\left[\frac{\mathrm{d} \ln A\left(x_{s}, y_{s}\right)}{\mathrm{d} s}\right] & =\mu+\left(x_{s}+\sigma^{2}\right)\left(1-\frac{a^{\prime}}{a}\right)+\frac{1}{2} \sigma^{2} \frac{a^{\prime \prime} a-a^{\prime 2}}{a^{2}}, \\
\operatorname{Var}\left[\frac{\mathrm{d} \ln A\left(x_{s}, y_{s}\right)}{\mathrm{d} s}\right] & =\sigma^{2} \frac{a^{\prime 2}}{a^{2}},
\end{aligned}
$$

omitting for notational convenience the argument $x_{s}$ of the function a $\left(x_{s}\right)$. The drift increases in the variable $x_{s}$ for small $x_{s}$ and converges to $\mu$ as $x_{s} \rightarrow \infty$, while the volatility increases in $x_{s}$ for all $x_{s}$ and converges to $\sigma^{2}$ as $x_{s} \rightarrow \infty$.

2. The effect of a growth shock $\mathrm{d} x_{0}$ on the log cash flow $\ln y_{s}$ and the log asset value $\ln A\left(x_{s}, y_{s}\right)$ in the short term $(s=0)$ and the long term $(s=\infty)$ is:

\begin{tabular}{lll} 
Effect of growth shock $\mathrm{d} x_{0}$ & $s=0$ & $s=\infty$ \\
\hline $\mathrm{d}\left(\mathrm{E} \ln y_{s}\right) / \mathrm{d} x_{0}$ & 0 & 1 \\
$\mathrm{~d} \ln A\left(\mathrm{E} x_{s}, e^{\mathrm{E} \ln y_{s}}\right) / \mathrm{d} x_{0}$ & $0<\frac{a^{\prime}(x)}{a(x)}<1$ & 1 \\
\hline
\end{tabular}

We draw three conclusions from the table in Proposition 3. First, in the short run, the asset value is an order of magnitude more volatile than the cash flow in response to shocks in $x_{0}$. This explains the high short-run volatility in asset values compared to the low short-run volatility in cash flows. Second, the long-run effect on the cash flow and the asset value are both equal to unity. The equality of the long-run effects is a fundamental feature of this process; the equality of both effects to unity is due to our transformation of the unit of time from $t$ to $s$, such that the coefficient of mean-reversion $\theta$ is normalized to unity. Third, the response of the asset value is larger in the long run than in the short run. There is thus persistence in the growth of the asset value: an increase in the asset value today predicts a further increase in the near future. This is consistent with the standard property that asset prices should be Markovian in the following sense. The total initial return to an upward shock in the growth rate has two components: (i) the higher cash flow and (ii) the indirect return of the growth in the asset value. Together, both components should be equal to the return on capital, $\rho A\left(x_{0}, y_{0}\right)$. Initially, there is no effect on the cash flow (see the entry for $\mathrm{d}\left(\mathrm{E} \ln y_{0}\right) / \mathrm{d} x_{0}$ in part two of Proposition 3 ), so the full return must come from the second component: the growth of the asset value. Over time, the cash flow increases such that it accounts for an increasing share of the return on the asset; hence, the second component must decline over time. Eventually, 
the cash flow converges to its new steady-state level, as the growth rate mean-reverts in expectation to its long-run equilibrium value, while the indirect return vanishes as the initial shock yields no further growth of the cash flow.

\section{The optimal stopping boundary}

\section{$4.1{ }^{*}$ The option value}

This subsection characterizes the option value when opportunities to exercise the option are generated by an exogenous Poisson process with intensity $\lambda<\infty$. Moreover, we investigate the limit $\lambda \rightarrow \infty$. The method described in this subsection may thus be valuable to readers interested in solving similar American-type option valuation problems.

Recall from Section 2 that the opportunities to invest are collected in the set $\mathcal{T}_{\lambda}=$ $\left\{\tau_{1}, \tau_{2}, \ldots\right\}$. Analogous to equation (3.1) for the asset value $A\left(x_{s}, y_{s}\right)$, the option value $V_{\lambda}\left(x_{s}, y_{s}\right)$ of being able to invest in the asset at some future time $\tau \in \mathcal{T}_{\lambda}$ - conditional on the state variables $x_{s}$ and $y_{s}$ - equals the present value of the asset value of the project at the moment of investment $\tau$ minus the present value of the cost of investment

$$
V_{\lambda}\left(x_{s}, y_{s}\right):=\max _{\tau \in \mathcal{T}_{\lambda}} \mathrm{E}_{s}\left[e^{-\rho \tau}\left(A\left(x_{\tau}, y_{\tau}\right)-\rho^{-1}\right)\right]
$$

At each of the Poisson arrival times in the set $\mathcal{T}_{\lambda}=\left\{\tau_{1}, \tau_{2}, \ldots\right\}$, the decision-maker must decide whether to continue waiting or to use the opportunity to invest. Since the asset value does not depend explicitly on time, the decision to stop depends only on $\left(x_{\tau}, y_{\tau}\right)$ for all $\tau \in \mathcal{T}_{\lambda}$. Thus, the optimal policy specifies a (closed) stopping region $\mathcal{S}_{\lambda}$, such that the decision-maker will stop at the earliest possible opportunity $\tau$ for which $\left(x_{\tau}, y_{\tau}\right) \in \mathcal{S}_{\lambda}$ :

$$
\tau:=\min _{s \in \mathcal{T}_{\lambda}}\left\{s \geq 0:\left(x_{s}, y_{s}\right) \in \mathcal{S}_{\lambda}\right\}
$$

where by assumption the minimum of an empty set equals infinity. It is useful to define the (open) continuation region $\mathcal{C}_{\lambda}$ as the complement of $\mathcal{S}_{\lambda}$, such that $\mathcal{C}_{\lambda} \cup \mathcal{S}_{\lambda}=\mathbb{R} \times \mathbb{R}_{\geq 0}$. Hence,

$$
\begin{aligned}
& \mathcal{C}_{\lambda}=\left\{(x, y) \in \mathbb{R} \times \mathbb{R}_{\geq 0}: V_{\lambda}(x, y)>A(x, y)-\rho^{-1}\right\}, \\
& \mathcal{S}_{\lambda}=\left\{(x, y) \in \mathbb{R} \times \mathbb{R}_{\geq 0}: V_{\lambda}(x, y) \leq A(x, y)-\rho^{-1}\right\} .
\end{aligned}
$$

There is thus a two-way dependence between the value function $V_{\lambda}(x, y)$ and the partitioning of space: $\mathcal{C}_{\lambda}$ and $\mathcal{S}_{\lambda}$ determine $V_{\lambda}(x, y)$ and in turn $\mathcal{C}_{\lambda}$ and $\mathcal{S}_{\lambda}$ can be read off from $V_{\lambda}(x, y)$.

Analogous to equation (3.2), the option value $V_{\lambda}(x, y)$ satisfies the following Bellman equation:

$$
(\rho-\mathcal{L}) V_{\lambda}(x, y)=\lambda\left[A(x, y)-\rho^{-1}-V_{\lambda}(x, y)\right]^{+}, \quad \forall(x, y) \in \mathbb{R} \times \mathbb{R}_{\geq 0}
$$


where $X^{+}:=\max (X, 0)$ for all $X \in \mathbb{R}$ and where the operator $\mathcal{L}$ is defined in equation (3.2). The return on the option value is equal to the arrival rate $\lambda$ of opportunities to invest times the wealth gain obtained at the moment the option is exercised. This wealth gain is zero if $V_{\lambda}(x, y)>A(x, y)-\rho^{-1}$; in that case, investment does not (yet) pay off and it is better to wait for the arrival of another opportunity to invest. The wealth gain is weakly positive if $V_{\lambda}(x, y) \leq A(x, y)-\rho^{-1}$, in which case it is optimal to invest (or equivalently, $\left.(x, y) \in \mathcal{S}_{\lambda}\right)$. Our solution method is based on the value of waiting $W_{\lambda}(x, y)$, which is defined as the difference between the option value $V_{\lambda}(x, y)$ and the asset value minus the cost of investment (that is, $\left.A(x, y)-\rho^{-1}\right)$; i.e.

$$
W_{\lambda}(x, y):=V_{\lambda}(x, y)-A(x, y)+\rho^{-1}, \quad \forall(x, y) \in \mathbb{R} \times \mathbb{R}_{\geq 0}
$$

Hence, $W_{\lambda}(x, y)>0$ for any $(x, y) \in \mathcal{C}_{\lambda}$, while $W_{\lambda}(x, y) \leq 0$ for any $(x, y) \in \mathcal{S}_{\lambda}$; in the latter case, the decision-maker will invest as soon as an opportunity to invest presents itself. The value of waiting satisfies the Bellman equation ${ }^{10}$

$$
(\rho+\lambda-\mathcal{L}) W_{\lambda}(x, y)=1-y+\lambda W_{\lambda}^{+}(x, y)
$$

In this expression, $V_{\lambda}(x, y)$ and $A(x, y)$ no longer appear, while the value waiting $W_{\lambda}(x, y)$ and the infinitesimal value of waiting $1-y$, which are arguably more fundamental quantities, emerge. The infinitesimal value of waiting is the interest payment on the investment (unity) minus the foregone cash flow $(y)$.

Since each Poisson time presents an opportunity (but no obligation) to realize the asset, and since more opportunities are better than fewer, the value of waiting $W_{\lambda}(x, y)$ is non-decreasing in the Poisson intensity $\lambda$. We define $W(x, y)$ as the pointwise limit of $W_{\lambda}(x, y)$; that is,

$$
W(x, y):=\lim _{\lambda \rightarrow \infty} W_{\lambda}(x, y) .
$$

We have $W_{\lambda}(x, y) \leq W(x, y)$ for all $\lambda \in(0, \infty]$. Equation (4.6) implies that for $(x, y) \in \mathcal{S}_{\lambda}$, the value of waiting $W_{\lambda}(x, y)$ is negative almost everywhere (except on the line $y=1$ ). A negative value of waiting means that the decision-maker wants to invest, but is forced to wait because investing is not permitted in the interval between two Poisson investment opportunities. This implies that $\lambda W_{\lambda}^{-}(x, y)$, where $X^{-}=\max (-X, 0)$ for all $X \in \mathbb{R}$, can be usefully interpreted as a 'penalty term' associated with this restriction. This observation links our approach with the penalty literature; see e.g. Forsyth and Vetzal (2002). It has apparently gone unnoticed that this penalty term appears automatically if the decision-maker is given the opportunity to invest only at a set of Poisson arrival times, as in this paper. Since $W_{\lambda}$ is increasing (or at least non-decreasing) in $\lambda$, it follows that

\footnotetext{
${ }^{10}$ This follows immediately from the PDE for $V_{\lambda}(x, y)$ in equation $(4.4)$ by subtracting $(\rho-\mathcal{L}) A(x, y)=y$ from both sides and using $1=(\rho-\mathcal{L}) \rho^{-1}$ and $\left[A(x, y)-\rho^{-1}-V_{\lambda}(x, y)\right]^{+}=W_{\lambda}^{-}(x, y)=W_{\lambda}^{+}(x, y)-$ $W_{\lambda}(x, y)$.
} 
$\mathcal{S}_{\lambda}$ must be shrinking as $\lambda$ increases. Conversely, the continuation set $\mathcal{C}_{\lambda}$ is expanding in $\lambda$. This makes intuitive sense, since the decision-maker can afford to wait longer when opportunities to exercise the option arrive more frequently. Thus, we define the limiting stopping and continuation sets as follows:

$$
\mathcal{S}:=\lim _{\lambda \rightarrow \infty} \mathcal{S}_{\lambda}, \quad \mathcal{C}:=\lim _{\lambda \rightarrow \infty} \mathcal{C}_{\lambda}
$$

where $\mathcal{S}$ is closed and $\mathcal{C}$ is its (open) complement.

\section{Proposition 4}

1. In the limit $\lambda \rightarrow \infty$, we obtain $\mathcal{S}=\left\{(x, y) \in \mathbb{R} \times \mathbb{R}_{\geq 0}: W(x, y)=0\right\}$ and $\mathcal{C}=\left\{(x, y) \in \mathbb{R} \times \mathbb{R}_{\geq 0}: W(x, y)>0\right\}$.

2. For $\lambda=\infty$, a sufficient condition for continuation and a necessary condition for stopping are as follows:

$$
\begin{aligned}
& 1-y>0 \Rightarrow(x, y) \in \mathcal{C}, \\
& (x, y) \in \mathcal{S} \Rightarrow 1-y \leq 0 .
\end{aligned}
$$

3. In the limit $\lambda \rightarrow \infty$, the penalty term $\lambda W_{\lambda}^{-}(x, y)$ converges pointwise as follows:

$$
\lambda W_{\lambda}^{-}(x, y) \rightarrow \begin{cases}0, & (x, y) \in \mathcal{C} \\ y-1 \geq 0, & (x, y) \in \mathcal{S} \backslash \partial \mathcal{S}\end{cases}
$$

where $\partial \mathcal{S}$ denotes the boundary of the (closed) set $\mathcal{S}$.

Part one states that when $\lambda$ goes to infinity, the decision-maker either waits (because $W(x, y)>0$ ) or invests immediately (in which case $W(x, y)=0$ ). Hence, $W(x, y) \geq 0$, since if $W(x, y)<0$, she would invest immediately, thereby setting $W(x, y)=0$. Note that for finite values of $\lambda, W_{\lambda}(x, y)$ can be negative in between two opportunities to invest. Part two says that the decision-maker will never invest when $1-y>0$ (that is: when the interest paid on the investment (unity) exceeds the rent on the investment $(y))$. Note, however, that this does not mean that the decision-maker builds as soon as $y=1$. Rather, she waits until $1-y$ is sufficiently negative before making the irreversible investment decision. Clearly, this implies that in the stopping region $\mathcal{S}$ we must have $1-y \leq 0$. Thus, as is standard in optimal stopping (see e.g. Øksendal (2007) p. 217-18), we have a sufficient condition for continuation $(1-y>0)$ and a necessary condition for stopping $(1-y \leq 0)$, where neither is both necessary and sufficient.

Part three gives an interpretation of the limit $\lambda \rightarrow \infty$ of the differential equation (4.6) for $W_{\lambda}(x, y)$. By subtracting $\lambda W_{\lambda}(x, y)$ from both sides, we may write

$$
(\rho-\mathcal{L}) W_{\lambda}(x, y)=1-y+\lambda W_{\lambda}^{-}(x, y)
$$


where $W_{\lambda}^{+}-W_{\lambda}=W_{\lambda}^{-}$. The third term on the right-hand side can be viewed as a 'penalty term', which penalizes the decision-maker whenever the value of waiting is negative. The 'strength' of this penalty increases as $\lambda$ increases, such that the limiting function $W(x, y)$ is forced to be non-negative (a strictly negative part of $W(x, y)$ would attract an infinity penalty). Thus, $W_{\lambda}^{-}(x, y)$ must approach zero as $\lambda \rightarrow \infty$. However, the fact that $W_{\lambda}^{-}(x, y)$ goes to zero says nothing about the limiting behavior of the penalty term $\lambda W_{\lambda}^{-}(x, y)$, which may still diverge to infinity. Part three of the proposition establishes that the limit of the penalty term $\lambda W_{\lambda}^{-}(x, y)$ is, in fact, bounded on bounded sets and converges pointwise on $\mathbb{R} \times \mathbb{R}_{\geq 0} \backslash \partial \mathcal{S}$. This result will be sufficient for our purposes under the relatively mild assumption that $\partial \mathcal{S}$ is of measure zero, where $\partial \mathcal{S}$ denotes the boundary of the set $\mathcal{S}$. That is, as long as the boundary $\partial \mathcal{S}$ is 'thin', we do not need to investigate the asymptotics of $\lambda W_{\lambda}^{-}(x, y)$ on $\partial \mathcal{S}$, which appears to be a difficult exercise.

Proposition 5 Assume that $\partial \mathcal{S}$ has Lebesgue measure zero. Then $W_{\lambda}(x, y)$ converges uniformly on compact sets to a limiting function $W(x, y):=\lim _{\lambda \rightarrow \infty} W_{\lambda}(x, y)$, which satisfies

$$
W(x, y)=\int_{0}^{\infty} \mathrm{e}^{-\rho s} \mathrm{E}_{(x, y)}\left[\mathbb{1}_{\mathcal{C}}\left(x_{s}, y_{s}\right)\left(1-y_{s}\right)\right] \mathrm{d} s,
$$

for all $(x, y) \in \mathbb{R} \times \mathbb{R}_{\geq 0}$, where $\mathbb{1}_{\mathcal{C}}(x, y)$ denotes the indicator function ${ }^{11}$ of the set $\mathcal{C}$. The limiting function $W\left(x_{0}, y_{0}\right)$ is continuously differentiable in $x_{0}$, but merely continuous in $y_{0}$ (i.e. the classic principle of smooth fit does not hold true in the $y$ direction).

Interestingly, Proposition 5 - the main result of this section - says that $W(x, y)$ equals the expected net present value of the infinitesimal value of waiting, that is $1-y_{s}$, which is received conditional on $\left(x_{s}, y_{s}\right) \in \mathcal{C}$. Hence, the infinitesimal value of waiting is realised only in the continuation region $\mathcal{C}$, which makes intuitive sense. The classic condition of 'value matching' is automatically satisfied, that is $W(x, y)=0$ for $(x, y) \in \mathcal{S}$. However, another classic condition known as 'smooth fit' is violated. Equation (4.11) implies that $W(x, y)$ is merely continuous in $y$, rather than once continuously differentiable as required by smooth fit. In one spatial dimension, it is well known that the principle of smooth fit breaks down when the underlying process is deterministic or when the underlying pay-off is non-smooth at the exercise boundary (see e.g. Alvarez (2001)). However, this paper seems to be the first to document the failure of smooth fit in higher dimensions. The reason is novel as well: smooth fit breaks down because the two-dimensional stochastic process is driven by a single source of uncertainty, implying it is degenerate. ${ }^{12}$ Intuitively, the the component $y_{s}$ is locally deterministic (i.e. when viewed on time scales of order $\mathrm{d} s)$.

\footnotetext{
${ }^{11}$ The indicator function $\mathbb{1}_{\mathcal{C}}(x, y)$ is equal to one if $(x, y) \in \mathcal{C}$ and zero otherwise.

${ }^{12}$ In the endnotes to their paper, Kwon and Lippman (2011) speculate that degenerate processes in higher dimensions may cause smooth fit to fail; hence, we confirm their conjecture in two dimensions.
} 


\subsection{Investment boundary $y(x)$}

For $\lambda=\infty$, the boundary of the stopping region $\mathcal{S}$ satisfies some special conditions. Define $y(x)$ to be the minimum value of the normalized cash flow $y$ for which investment is optimal conditional on the modified growth rate $x$; any point for which $y \geq y(x)$ is therefore part of the stopping region: $y \geq y(x) \Leftrightarrow(x, y) \in \mathcal{S}$. Hence, $\partial \mathcal{S}=y(x)$. The boundary of the stopping region $y(x)$ satisfies two necessary conditions, both of which have a direct economic interpretation. The first condition is $y(x)>1$ (see equation (4.8)): if the reverse is true - the interest payment exceeds the cash flow — it is always better to postpone investment. The second condition is that the asset value must be greater than the cost of investment; otherwise, the investor would be worse off by making the investment. Hence, $\rho A[x, y(x)]=\rho a(x) y(x)>1$. We do not have an explicit solution for this equation, but Proposition 2 shows it converges to a linear relation between $y$ and $x$ as $x \rightarrow-\infty$ :

$$
\rho y(x) \geq a(x)^{-1}=\psi-\frac{1}{2} \sigma^{2}-x+O\left(x^{-1}\right) .
$$

The conditions (4.8) and (4.12) are necessary but not sufficient. If the cash flow only just covers the interest on the investment, then it is better to delay investment (since the stochastic law of motion of $(x, y)$ might move the system back to the subset $\mathcal{C})$. Similarly, if the expected present value of the cash flow only just covers the cost of investment, then it is better to delay (again because the system might move back to $(x, y) \in \mathcal{C}$ ). Hence, the function $y(x)$ must be strictly north of the curves $y(x)>1$ and $\rho a(x) y(x)>1$. The stopping region $\mathcal{S}$ is therefore a subset of the convex subset of the $(x, y)$ space that is delimited by these conditions. We are unable to derive explicit statements about the shape of $y(x)$ and hence about $A[x, y(x)]$. However, the arguments above suggest that the following conjecture holds.

Conjecture 1 The investment boundary $y(x)$ for the Leontief case satisfies the following:

$$
\begin{array}{ccc}
1 & : & y^{\prime}(x)<0, \quad y^{\prime \prime}(x)>0 ; \\
2 & : & \lim _{x \rightarrow \infty} y(x)=1 ; \\
3 & : & \lim _{x \rightarrow \infty} A[x, y(x)]=\lim _{x \rightarrow \infty} a(x)=\infty ; \\
4 & : & \lim _{x \rightarrow-\infty} \rho A[x, y(x)]=\lim _{x \rightarrow-\infty} \frac{\rho}{\psi-\frac{1}{2} \sigma^{2}-x} y(x)>1 ; \\
5 & : & \frac{\mathrm{d} A[x, y(x)]}{\mathrm{d} x}>0 .
\end{array}
$$

Part one is motivated by the fact that conditions (4.8) and (4.12) rule out that $y(x)$ is a straight line, since this would violate at least one of the necessary conditions. Moreover, both conditions imply that (assuming that $y(x)$ is twice differentiable) $y^{\prime}(x)$ must be negative and $y^{\prime \prime}(x)$ positive for at least some values of $x$. Part two is motivated by the fact that for high growth rates, the only reason for an investor to postpone investment is that the current net cash flow is negative. As soon as $y$ has grown to 1 (the interest 
payment on the investment), it is optimal to invest because high growth ensures the net cash flow will grow afterwards. An immediate implication of this conjecture is that when the growth rate tends to infinity, the asset value at the moment of investment is infinite (part three). Part four states that for very low growth rates $y(x)$ converges to condition (4.12). The option value is zero at that point because the negative growth implies that the project will move to the south-east in the state space $(x, y)$, driving it away from the investment boundary $y(x)$; since waiting makes things worse, it is optimal to invest as soon as the net present value of the project is positive. ${ }^{13}$ For that reason, the cost of investment $\rho^{-1}$ provides a lower bound for the asset value along the investment boundary $A[x, y(x)]$. This lower bound will be attained for low growth rates $x$. Part five generalizes the ideas in parts three and four by conjecturing that $A[x, y(x)]$ is increasing in $x$. Though we cannot prove this conjecture, our numerical analysis (presented below) supports it consistently. Notice that this conjecture applies for $\lambda=\infty$ only. For finite values of $\lambda, y_{\lambda}(x)$ will cross the horizontal line $y=1$ for high values of $x$ because it is optimal to use the opportunity to invest even when the net cash flow is still negative (since it might take some time for the next investment opportunity to arrive and the cash flow $y$ will almost certainly exceed unity shortly).

\section{$4.3 *$ Numerical implementation}

For the numerical solution to the option value problem we use the value of waiting $W_{\lambda}(x, y)$ as defined in equation (4.6). The advantage of this approach is that both $V_{\lambda}(x, y)$ and $A(x, y)$ disappear from our expressions and are replaced by a single function $W_{\lambda}(x, y)$. This is convenient, since we have no closed-form solution for $A(x, y)$. The PDE for the value of waiting is particularly useful in that we can generate a sequence of lower bounds for $W_{\lambda}(x, y)$ which converge monotonically to the true value.

Proposition 6 The recursively defined sequence of functions

$$
\begin{aligned}
(\rho+\lambda-\mathcal{L}) W_{\lambda}^{(1)}(x, y) & :=1-y, \\
(\rho+\lambda-\mathcal{L}) W_{\lambda}^{(n)}(x, y) & :=1-y+\lambda\left[W_{\lambda}^{(n-1)}(x, y)\right]^{+}, \quad n \geq 2,
\end{aligned}
$$

is monotonically increasing and (at least pointwise) convergent to a limiting function $W_{\lambda}(x, y)$, which is bounded above by $\rho^{-1}$.

\footnotetext{
${ }^{13}$ Note that $\mathrm{E}\left[\frac{\mathrm{d} y}{\mathrm{~d} s}\right]=\left(x+\mu^{2}+\sigma^{2}\right) y$ and $\mathrm{E}\left[\frac{\mathrm{d} x}{\mathrm{~d} s}\right]=-\left(x+\sigma^{2}\right)$. Hence, the expected drift for $x \rightarrow-\infty$ is $\lim _{x \rightarrow-\infty} \frac{\mathrm{d} y}{\mathrm{~d} x}=\lim _{x \rightarrow-\infty}-\frac{\left(x+\mu^{2}+\sigma^{2}\right) y}{\left(x+\sigma^{2}\right)}=-y$, while the slope of condition (4.12) is $-\rho^{-1}$. Hence, for $y(x)>\rho^{-1}$ (that is: for large negative values of $x$ ), the south-bound drift in $(x, y)$ will exceed the negative slope of $y(x)$ and hence the drift will move $(x, y)$ further into the continuation region $\mathcal{C}$.
} 
This is, to the best of our knowledge, a novel solution method. ${ }^{14}$ It also has an interesting economic interpretation. A lower bound $W_{\lambda}^{(1)}(x, y)$ of the value waiting at some Poisson time $\tau_{k}$ for some $k \in \mathbb{N}$ is the expected discounted value of the cost of investment minus the expected cash flow, $(\rho+\lambda-\mathcal{L})^{-1}(1-y)$, until the next investment opportunity $\tau_{k+1}$. However, a higher and more precise estimate of the value of waiting, $W_{\lambda}^{(2)}(x, y)$, takes into account the net expected value of the investment cost minus the cash flow plus the additional value of waiting until the next investment opportunity $\tau_{k+1}$ (provided that this additional value is positive). Thus, $W_{\lambda}^{(2)}(x, y) \geq W_{\lambda}^{(1)}(x, y)$. The difference operator $\mathcal{L}$ implies that an increase in $W_{\lambda}^{(n)}(x, y)$ from $n$ to $n+1$ also increases $W_{\lambda}^{(n+1)}(x \pm \mathrm{d} x, y \pm \mathrm{d} y)$ for small displacements $\mathrm{d} x$ and $\mathrm{d} y$. The value of waiting at a particular point $(x, y)$ in the state space is an increasing function of the value of waiting in neighboring areas in the state space, since the stochastic dynamics of $(x, y)$ might move the system to these areas. Thus, every subsequent iteration accounts for a higher value of waiting at the next investment opportunity $\tau_{k+1}$, which in turn increases the value of waiting at the current investment opportunity $\tau_{k}$. However, even though the value of $W_{\lambda}^{(n)}(x, y)$ is increasing in $n$, it remains bounded for $n \rightarrow \infty$. In fact, it can never exceed the present value of investment, $\rho^{-1}$, because the largest gain that can be obtained by postponing investment is the cost of the investment itself.

While Proposition 6 gives the convergence of $W_{\lambda}(x, y)$ in function space, we must work in some finite-dimensional vector space for a numerical analysis. Thus we discretize the state space $(x, y) \in \mathbb{R} \times \mathbb{R}_{\geq 0}$ using $N$ equidistant grid points for both $x$ and $y$ on some bounded region of interest. ${ }^{15}$ Discretization converts the function $W_{\lambda}(x, y)$ on $\mathbb{R} \times \mathbb{R}_{\geq 0}$ into a matrix of dimension $N \times N$ denoted $\mathbf{W}_{\lambda}$, where we adopt the convention to denote all matrices in bold. Define $\underline{W}_{\lambda}:=\operatorname{Vec}\left[\mathbf{W}_{\lambda}\right]$; all vectors will be underlined. Hence, the vector $\underline{W}_{\lambda}$ has length $N^{2}$. Similarly, discretizing the differential operator $\mathcal{L}$ yields an $N^{2} \times N^{2}$ matrix, denoted by $\mathbf{L}$. The discretized system has the same form as the PDE $(4.6)$ :

$$
[(\rho+\lambda) \mathbf{I}-\mathbf{L}] \underline{W}_{\lambda}=\underline{1}-\underline{y}+\lambda \underline{W}_{\lambda}^{+},
$$

where $\mathbf{I}$ denotes the $N^{2} \times N^{2}$ identity matrix, $\underline{1}$ is a vector of ones of length $N^{2}$ and $\underline{y}:=\operatorname{Vec}\left[\underline{y}^{(N)} \underline{1}^{(N) \prime}\right]$, and $\underline{1}^{(N)}$ and $\underline{y}^{(N)}$ denote vectors of length $N$ (hence $\underline{y}$ has length $N^{2}$ ). The discretized problem (4.15) suggests an iterative solution method that is analogous to the iterative method (4.14) in function space:

$$
[(\rho+\lambda) \mathbf{I}-\mathbf{L}] \underline{W}_{\lambda}^{(n)}=\underline{1}-\underline{y}+\lambda\left[\underline{W}_{\lambda}^{(n-1)}\right]^{+}, \quad n \geq 1
$$

with $\underline{W}_{\lambda}^{(0)}=\underline{0}$.

\footnotetext{
${ }^{14} \mathrm{~A}$ related proposition appears in Lange et al. (in prep) in the context of more general stochastic processes, such as Hunt and Lévy processes.

${ }^{15}$ The method can be generalized by allowing $N_{x} \neq N_{y}$ and by making the gridpoints not equidistant.
} 


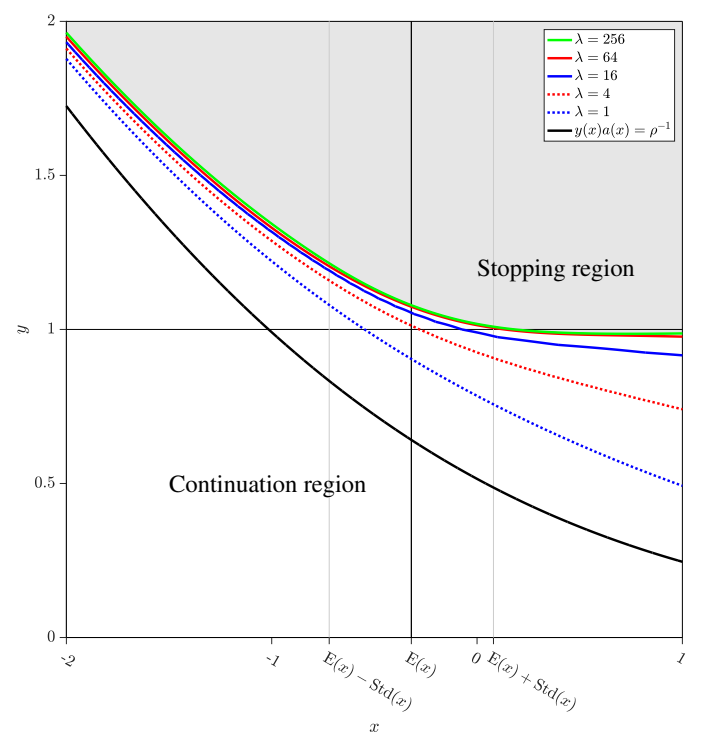

Figure 3: The exercise boundaries $y_{\lambda}(x)$ and the conditions $y>1$ and $y>[\rho a(x)]^{-1}$ for various values of $\lambda$.

Proposition 7 For an appropriately discretization $\mathbf{L}$, the iterative method (4.16) results in a non-decreasing sequence of finite-dimensional vectors $\left\{\underline{W}_{\lambda}^{(n)}\right\}_{n \in \mathbb{N}}$ that converge to a limiting vector $\underline{W}_{\lambda}$, which solves (4.15).

The appropriate discretization of the differential operator $\mathcal{L}$ that gives rise to the matrix $\mathbf{L}$ requires us to think carefully about forward and backward finite differences, and is discussed in Appendix A.10. The proposed numerical method is stable and converges quickly.

\subsection{Results}

This subsection presents our numerical results for the benchmark parameters (2.3) and various values of the Poisson intensity $\lambda$.

Figure 3 shows the investment boundaries $y(x)$ for the benchmark parameters for various values of $\lambda$, ranging from $\lambda=1$ (since $\theta=0.05$, a unit of time on the transformed time scale is 20 years; hence, $\lambda=1$ corresponds to one opportunity to invest every 20

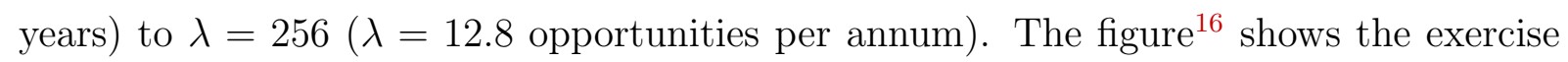
curves $y(x)$ as well as the conditions for $(x, y) \in \mathcal{S}_{\lambda}$ (equation (4.8) $y(x)>1$ and equation (4.12) $\left.y(x)>[\rho a(x)]^{-1}\right)$.

Our calculations confirm that $y_{\lambda}(x)$ is increasing in $\lambda$ (see equation (4.7)): the more frequent the opportunities to invest, the less costly it is to wait for the next opportunity

\footnotetext{
${ }^{16}$ For each value of $\lambda$, we performed the calculation on a $501 \times 401$ grid, ranging from $x=-4.5$ to $x=5.5$ and $y=0$ to $y=10$ (implying step sizes $\Delta_{x}=0.02$ and $\Delta_{y}=0.025$ ). Note that only a subset of the range used for our calculation is displayed. Experiments with larger grids and different boundary conditions on the edge of the grid gave nearly identical results.
} 


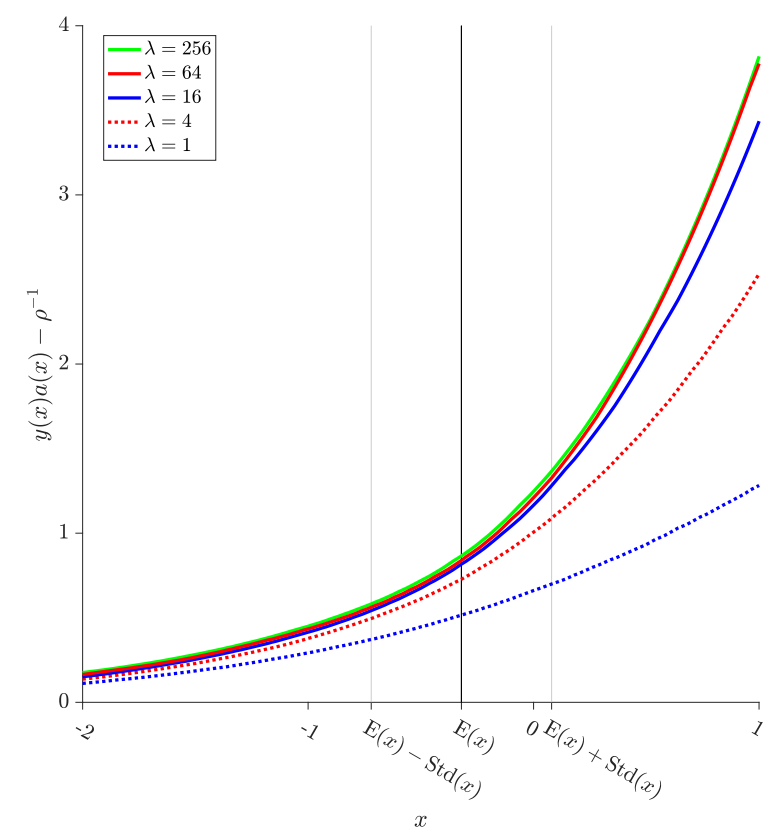

Figure 4: The option value $V(x, y)$ for $y=y_{\lambda}(x)$ for various values of $\lambda$.

(and hence the larger the continuation region $\mathcal{C}_{\lambda}$ ). Also, the calculations confirm that $y_{\lambda}(x)<1$ for low values of $\lambda$ and high values of $x$ : investors invest even though the net cash flow is still negative, since it is more costly to wait for the next opportunity to invest (as the cash flow will grow in the meantime). Finally, the results are consistent with Conjecture 1. For $x \rightarrow \infty, y(x)$ converges to $y=1$. Similarly, for $x \rightarrow-\infty, y_{\lambda}(x)$ converges to condition (4.12), although Figure 3 reveals that the convergence is slow. The curves $y_{\lambda}(x)$ for $\lambda=64$ and $\lambda=256$ are almost indistinguishable, suggesting that these curves closely approximate the limiting function $y(x)$.

Figure 4 shows the asset value $V_{\lambda}(x, y)$ along the exercise boundary $y=y_{\lambda}(x)$ as a function of the growth rate of the cash flow $x$. Again, the graphs for $\lambda=64$ and $\lambda=256$ are practically indistinguishable. All calculations for subsequent figures therefore use $\lambda=$ 256. For this reason we henceforth drop the subscript $\lambda$. Figure 4 shows that the option value is strongly increasing as a function of the growth rate at the moment the option is exercised, consistent with part five of Conjecture 1 (since $V[x, y(x)]=A[x, y(x)]-\rho^{-1}$ ).

Figure 5 shows the same asset value $V(x, y)$, but now as a function of the level of the cash flow $y$. For $y \geq y(x), V(x, y)=A(x, y)-\rho^{-1}=a(x) y-\rho^{-1}$, which is linear in $y$. Irrespective of the value of $x$ (and hence $a(x)$ ), these linear lines cross the vertical axis at the negative value $-\rho^{-1}=-1.25$; see equation (2.3). Since $a^{\prime}(x)>0$, the higher $x$, the steeper the line $a(x) y-\rho^{-1}$. For $0<y<y(x), V(x, y)>a(x) y-\rho^{-1}$. The point of departure of $V(x, y)$ from the straight lines $a(x) y-\rho^{-1}$ occurs at the exercise boundary $y(x)$. Since $y^{\prime}(x)<0$ and $a^{\prime}(x)>0$, this lowest point of tangency is decreasing in $x$. Finally, $V(x, 0)=0$ for all $x$, since the law of motion of $y$ in (2.1) implies that the 


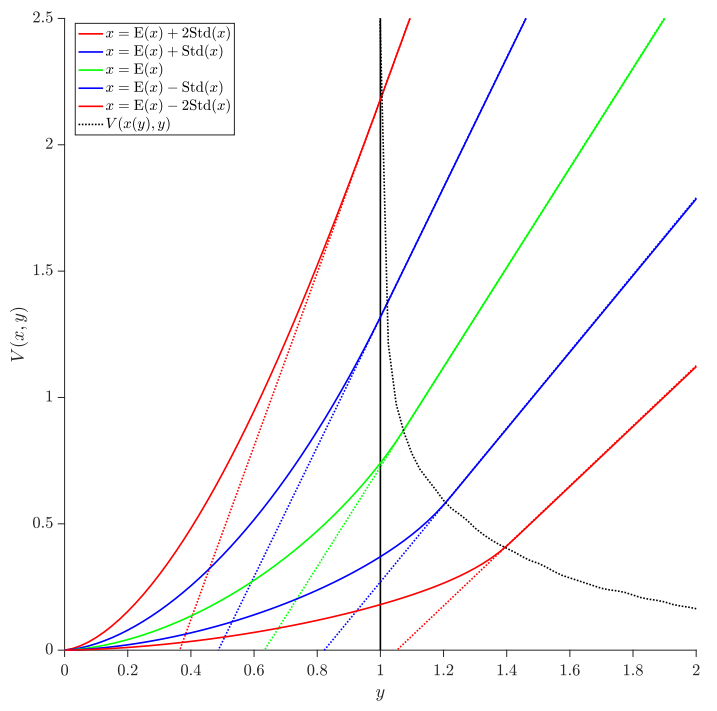

Figure 5: The option value $V(x, y)$ in the direction of $y$ for various values of $x$.

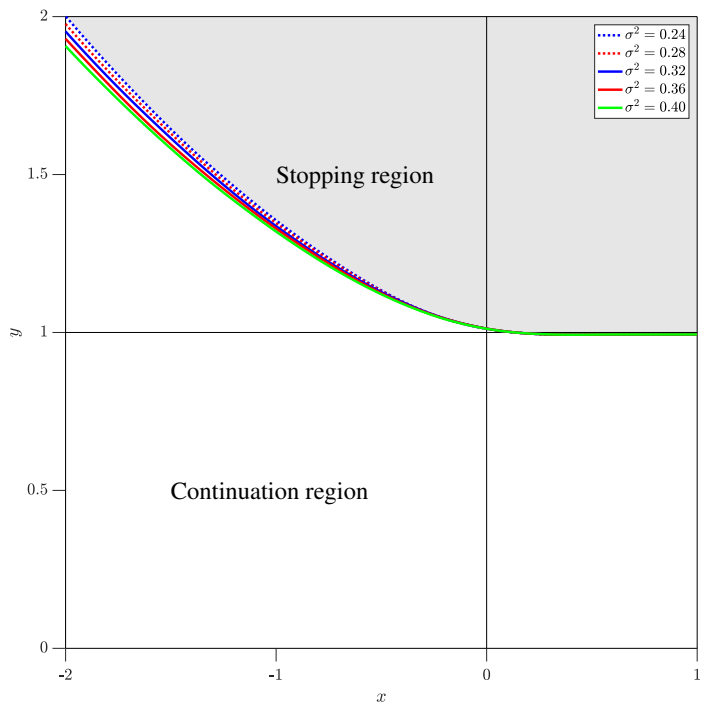

Figure 6: The exercise boundary $y(x)$ for various values of $\sigma^{2}$. 
potential cash flow will never depart from zero when its initial level is zero.

Finally, Figure 6 shows the exercise boundary $y(x)$ for various values of $\sigma^{2}$; this value has little bearing on the trajectory of $y(x)$. This conclusion is slightly deceptive due to our transformation of the parameters, which relate the annual growth rate $g_{t}$ to $x_{t}$ by $x_{t}:=g_{t} / \theta-\mu-\sigma^{2}$; see equation (2.2). A change in $\sigma^{2}$ therefore implies a mean shift in $x_{t}$ relative to the underlying growth $g_{t}$. This transformation of $g_{t}$ to $x_{t}$ absorbs most of the variation in the exercise boundary.

\section{Generalization to Stone-Geary}

\subsection{The model}

In this section we use the machinery developed in the previous section to extend our analysis to the Stone-Geary case. In the Stone-Geary case, the owner of the asset must make - on top of the fixed investment $\mathcal{I}$ - a variable investment $\mathcal{K}$ that enhances the level of the cash flow. Specifically, we assume:

Definition 3 The Stone-Geary production function is defined as

$$
\text { cash flow }=\phi \mathcal{K}^{\alpha} y
$$

where $\alpha \in[0,1)$ and

$$
\phi:=(1-\alpha)^{\alpha-1} \alpha^{-\alpha}, \quad \mathcal{I}:=\rho^{1 /(\alpha-1)} .
$$

As will be discussed below, the choices for $\phi$ and $\mathcal{I}$ are convenient normalizations. The Leontief case as in Section 2 is a special case of Definition 3, obtained by setting $\alpha=0$. Jointly, the fixed investment $\mathcal{I}=\rho^{1 /(\alpha-1)}$ and the variable investment $\mathcal{K}$ are irreversible in both downward and upward directions. The total investment is $\mathcal{K}+\rho^{1 /(\alpha-1)}$. In the context of a city, one can think of $y$ as the location premium of land at that particular location. In that context, there are several interpretations of the fixed investment $\rho^{1 /(\alpha-1)}$; it can be seen as either the return on the alternative use of the land for agriculture, or the fixed cost of construction that applies irrespective of the size of the building. In the context of a firm, one can think of $y$ as the TFP of that firm. We shall also consider the limiting case of a full Cobb-Douglas technology where all investment is variable. The elasticity of substitution between construction and land (or TFP for a firm) is equal to unity for Cobb-Douglas, zero for Leontief, and between zero and one for Stone-Geary. Empirically, the cost of construction usually constitutes some $60 \%$ of the total value of a building, which seemingly corresponds to $\alpha=0.60$; see Davis and Heathcote (2007) and Davis and Palumbo (2008). However, part of this $60 \%$ share of construction is the fixed investment $\rho^{1 /(\alpha-1)}$. Hence, the actual value of $\alpha$ must be less than 0.60 . As a fair 
compromise between the limiting cases of Leontief $(\alpha=0)$ and Cobb-Douglas (no fixed investment), we choose the following benchmark value for $\alpha$ :

Benchmark value: $\alpha=0.30$.

In this extended model, the owner of the option has to make two decisions: (i) at what time $\tau$ to invest, and (ii) how much to invest at that time, that is, what is the optimal level of $\mathcal{K}$ ? A straightforward extension of equation (3.1) puts the value of the project at time $s$ as

$$
B\left(x_{s}, y_{s}, \mathcal{K}\right):=\phi \mathcal{K}^{\alpha} \mathrm{E}_{s}\left[\int_{s}^{\infty} y_{t} \mathrm{e}^{-\rho(t-s)} \mathrm{d} t\right]=\phi \mathcal{K}^{\alpha} A\left(x_{s}, y_{s}\right)
$$

The optimal level of variable investment $\mathcal{K}$ maximizes this asset value minus the cost of investment

$$
\mathcal{K}^{*}(x, y):=\underset{\mathcal{K} \geq 0}{\arg \max }\left[\phi \mathcal{K}^{\alpha} A(x, y)-\mathcal{K}-\rho^{1 /(\alpha-1)}\right] .
$$

The maximization problem (5.4) can be solved in closed form to give

$$
\begin{aligned}
\mathcal{K}^{*}(x, y) & =[\alpha \phi A(x, y)]^{1 /(1-\alpha)} \\
B^{*}(x, y) & =\phi \mathcal{K}^{*}(x, y)^{\alpha} A(x, y)=\widetilde{\alpha}[\phi A(x, y)]^{1 /(1-\alpha)} \\
\widetilde{\alpha} & :=\alpha^{\alpha /(1-\alpha)}
\end{aligned}
$$

Equation (5.5) implies $\mathcal{K}^{*}(x, y)=\alpha B^{*}(x, y)$; i.e. the variable investment $\mathcal{K}^{*}(x, y)$ is a fixed share $\alpha$ of the optimal value of the project $B^{*}(x, y)$. This is the standard result for a Cobb-Douglas (or Stone-Geary) technology, where $\alpha$ is the share of total (or variable) investment in value added. Equation (5.5) also implies that the optimal value $B^{*}(x, y)$ is proportional to $A(x, y)$ raised to the power $(1-\alpha)^{-1} \geq 1$. The option to adjust the level of investment $\mathcal{K}$ to the state of the asset $(x, y)$ at the moment of investment implies that $B^{*}(x, y)$ is more sensitive to $(x, y)$ than is $A(x, y)$.

The increased sensitivity of $B^{*}(x, y)$ to the state variables $(x, y)$ implies that condition (3.4) is no longer sufficient for the option value to be bounded. Although (3.4) guarantees that $A(x, y)$ and thus $B^{*}(x, y)=\widetilde{\alpha}[\phi A(x, y)]^{1 /(1-\alpha)}$ is bounded for $(x, y)$ in bounded sets, this condition is insufficient to guarantee that the option value is bounded. The option value is an expectation, and $\mathrm{E} B^{*}(x, y)$ need not be bounded even if the function under the expectation sign is bounded on bounded sets. By equation (2.7), $\ln y_{s}-\ln y_{0}$ is normally distributed with a long-run expectation $\mathrm{E}\left[\ln y_{s}-\ln y_{0}\right]=s \mu$ and variance $\operatorname{Var}\left[\ln y_{s}-\ln y_{0}\right]=s \sigma^{2}$. Using the formula for the expectation of the log normal distribution, the long-run growth rate of $y^{1 /(1-\alpha)}$ is $(1-\alpha)^{-1} \mu+\frac{1}{2}(1-\alpha)^{-2} \sigma^{2}$. This growth rate must be smaller than the discount rate $\rho$ for the value of the option to invest in $B^{*}(x, y)$ 
to be bounded for $(x, y)$ in bounded sets. Hence, we require ${ }^{17}$

$$
\begin{aligned}
\rho & >(1-\alpha)^{-1} \mu+\frac{1}{2}(1-\alpha)^{-2} \sigma^{2} \Rightarrow \\
\alpha & <1-\frac{\mu}{2 \rho}-\sqrt{\left(\frac{\mu}{2 \rho}\right)^{2}+\frac{\sigma^{2}}{2 \rho}},
\end{aligned}
$$

which is a stricter condition than (3.4). In what follows we assume (5.6) to be satisfied. For our benchmark parameters, condition (5.6) implies that we need $\alpha<0.41$ for the option value to be bounded; our benchmark value $\alpha=0.30$ satisfies this constraint.

Finally, we provide the rationale for the normalization of $\phi$ and $\mathcal{I}$; see equation (5.2). We normalize our unit of currency such that $1=\rho \mathcal{I}^{1-\alpha}$ (recall that $1=\rho \mathcal{I}$ in the Leontief case), such that $\mathcal{I}=\rho^{1 /(\alpha-1)}$. For Leontief, $y \geq 1$ was a necessary condition for the investor to recover his interest payment $\rho \mathcal{I}$ on the fixed investment $\mathcal{I}$ (since $\mathcal{I}=\rho^{-1}$ for Leontief). For Stone-Geary, $\phi$ is defined such that the same necessary condition applies: for $y=1$, the cash flow $\phi y \mathcal{K}^{*}(x, y)^{\alpha}$ is (weakly) insufficient to cover the interest payment on the total (fixed and variable) investment for any value of $x \in \mathbb{R}$. Hence for $y=1$ the cash flow satisfies

$$
\phi \mathcal{K}^{*}(x, 1)^{\alpha} \leq \rho \mathcal{K}^{*}(x, 1)+\rho^{\alpha /(\alpha-1)}, \quad \forall x \in \mathbb{R}
$$

where we use equation (5.2) to substitute for $\mathcal{I}$. Since $x$ appears only in $\mathcal{K}^{*}(x, 1)$, this inequality must hold for all values of $\mathcal{K}:=\mathcal{K}^{*}(x, 1) \in(0, \infty)$. Thus, $\phi$ solves

$$
\begin{aligned}
\rho^{\alpha /(\alpha-1)} & =\max _{\mathcal{K}>0}\left[\phi \mathcal{K}^{\alpha}-\rho \mathcal{K}\right]=\phi\left(\frac{\alpha \phi}{\rho}\right)^{\alpha /(1-\alpha)}-\rho\left(\frac{\alpha \phi}{\rho}\right)^{1 /(1-\alpha)} \\
& =\left(\rho^{-\alpha} \alpha^{\alpha} \phi\right)^{1 /(1-\alpha)}(1-\alpha)
\end{aligned}
$$

Solving this equation for $\phi$ yields the expression for this parameter in equation (5.2). For the benchmark value $\alpha=0.30$, this implies $\phi \approx 1.842$.

\subsection{Cobb-Douglas}

For a Cobb-Douglas production function, in which case $\mathcal{I}=0$, the normalization $1=$ $\rho \mathcal{I}^{1-\alpha}$ is undefined. Since the normalization is arbitrary in this case, the pragmatic choice is simply to ignore the fixed cost $\rho^{\alpha /(\alpha-1)}$. The Cobb-Douglas case can be solved analytically for the limiting case $\lambda=\infty$, which is of interest in this section. We use the Bellman equations (4.4) to write

$$
\begin{aligned}
& (\rho-\mathcal{L}) V(x, y)=0, \quad \forall(x, y) \in \mathcal{C}, \\
& (\rho-\mathcal{L}) A(x, y)=y, \quad \forall(x, y) \in \mathbb{R} \times \mathbb{R}_{\geq 0}, \\
& (1-\alpha) B^{*}(x, y)=V(x, y), \quad \forall(x, y) \in \partial \mathcal{S} .
\end{aligned}
$$

\footnotetext{
${ }^{17}$ In the second line, only the lower root for $\alpha$ is relevant, since the upper root is greater than unity.
} 
The first two lines are the Bellman equations for the option value and the realized project, while the third line gives the value-matching condition: at the moment of investment, the option value must be equal to the value of the project after investment $\phi \mathcal{K}^{*}(x, y) A(x, y)$ minus the cost of the (variable) investment $\mathcal{K}^{*}(x, y)$, which represents a share $\alpha$ of the value of the project. The Bellman equation for the optimally scaled project (that is, $\left.(1-\alpha) B^{*}(x, y)\right)$ reads

$$
\begin{aligned}
& (\rho-\mathcal{L})(1-\alpha) B^{*}(x, y)= \\
& \quad \phi y[\alpha \phi y a(x)]^{\alpha /(1-\alpha)}\left[1-\rho \alpha a(x)-\frac{\sigma^{2}}{2} \frac{\alpha}{1-\alpha} \frac{a^{\prime}(x)^{2}}{a(x)}\right],
\end{aligned}
$$

which follows from the chain rule and the Bellman equation for $A(x, y)$ given above (see Appendix A.11 for the derivation). For $\alpha=0$ we recover the Bellman equation for $A(x, y)$. The pre-factor on the right-hand side of (5.8) is recognized as $\phi y \mathcal{K}^{*}(x, y)^{\alpha}$, while the terms inside square brackets, which play a prominent role in the next Proposition, account for the received cash flow, the cost of the interest payment on the variable investment, and the opportunity cost of fixing $\mathcal{K}$, which can no longer be changed after the moment of investment.

Proposition 8 The following statements hold for the exercise boundary for a CobbDouglas production function:

1. The optimal moment to invest does not depend on the current level of the cash flow $y$.

2. Investment is always optimal when $x \leq x^{*}$ and is never optimal when $x>x^{*}{ }^{18}$ The critical threshold $x^{*}$ is less than or equal to $x^{o}$, where $x^{o}$ solves

$$
0=1-\rho \alpha a\left(x^{o}\right)-\frac{\sigma^{2}}{2} \frac{\alpha}{1-\alpha} \frac{a^{\prime}\left(x^{o}\right)^{2}}{a\left(x^{o}\right)} .
$$

The solution $x^{o}$ to equation (5.9) is unique, finite, and decreasing in $\alpha$.

Proposition 8 yields two counterintuitive conclusions. First, the optimal moment of investment does not depend on the current level of the cash flow $y$. The intuition for this result is that the optimal investment is proportional to $y$. Regardless of the value of $y$, the optimal investment is always a fixed multiple $\frac{\alpha}{1-\alpha}$ of the option value. Because any change in $y$ will affect both the cost and the benefit of investment proportionally, the optimal moment to invest does not depend on $y$. Second, investment should be postponed for high rather than for low levels of the growth rate $x$. By postponing investment, the investor (i) saves the interest payments on the investment, and (ii) benefits from the additional

\footnotetext{
${ }^{18}$ In our standard notation, $\mathcal{S}=\left\{x \in \mathbb{R}: x \leq x^{*}\right\}$ and $\mathcal{C}=\left\{x \in \mathbb{R}: x>x^{*}\right\}$.
} 


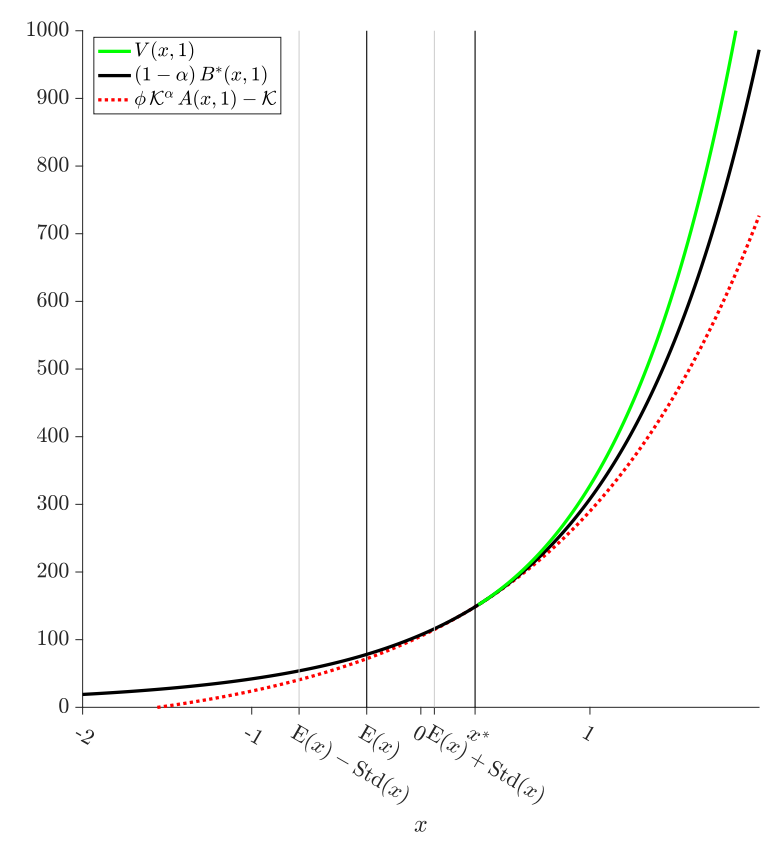

Figure 7: The values $V(x, 1),(1-\alpha) B^{*}(x, 1)$, and $\mathcal{K}^{\alpha} A(x, 1)$ as a function of $x$.

information on the evolution of $(x, y)$ that comes to light during the period of delay, enabling her to tailor any future investment to this new information. The right-hand side of equation (5.9) captures the cash flow minus the interest bill on the investment, both scaled per unit of $\phi y \mathcal{K}^{*}(x, y)^{\alpha}$. For a high growth rate, the interest bill might exceed the cash flow, since the optimal investment accounts for the future growth in $y$ and therefore exceeds the level that is efficient for the current level of $y$. The final term is the opportunity cost of fixing $\mathcal{K}$ : any additional information received during the interval $\mathrm{d} s$ allows the investor to adapt the level of investment to the evolution of $(x, y)$. One therefore expects the threshold $x^{o}$ to decrease as a function of $\alpha, \rho$ and $\sigma^{2}$ : a higher share of investment, a higher interest rate, and greater uncertainty make it more attractive to postpone investment. ${ }^{19}$

In Appendix A.13 we provide a numerically simple solution method for computing the exact threshold $x^{*}$ as the solution to (a non-standard version of) Hermite's differential equation. This equation has two independent solutions, both of which are confluent hypergeometric functions. The boundary condition at positive infinity prescribes that we take a particular linear combination, such that only one constant remains. The constant can be found in conjunction with $x^{*}$ by applying the standard principles of value matching and smooth pasting.

The proof of Proposition 8 has an interesting economic interpretation. Consider three asset values displayed in Figure 7 for $y=1$ :

\footnotetext{
${ }^{19}$ This proof is simple for $\alpha$. For $\rho$ and $\sigma^{2}$ we provide no formal proof, since these parameters also affect the function $a(x)$.
} 
1. the option value of investing in the project at any future time, $V(x, y)$;

2. the value of the project minus the cost of investment, where the investment is set at today's optimal level $\mathcal{K}^{*}(x, y)=\alpha B(x, y)$, that is, $(1-\alpha) B^{*}(x, y)$;

3. the value of the project at some arbitrary, not necessarily optimal, level $\mathcal{K}$; again minus of the cost of investment, $\phi \mathcal{K}^{\alpha} A(x, y)-\mathcal{K}$.

As illustrated in Figure 7, the following weak inequalities hold:

$$
V(x, y) \geq(1-\alpha) B^{*}(x, y) \geq \phi \mathcal{K}^{\alpha} A(x, y)-\mathcal{K}, \quad \forall(x, y) \in \mathbb{R} \times \mathbb{R}_{\geq 0}
$$

The option to invest in the project at any time is weakly larger than the option to invest today, since the option to invest at any time includes the option to invest today. Equality applies only in stopping zone $\mathcal{S}$ (that is, for $x \leq x^{*}$ ), in which case it is optimal to invest today. Likewise, the value of the project for the optimal level of investment in the current state is weakly larger than the value of the project at some arbitrary level of investment $\mathcal{K}$, since $(1-\alpha) B^{*}(x, y)=\max _{\mathcal{K}}\left[\phi \mathcal{K}^{\alpha} A(x, y)-\mathcal{K}\right]$. Hence, both are equal only for $\mathcal{K}=\arg \max _{\mathcal{K}}\left[\phi \mathcal{K}^{\alpha} A(x, y)-\mathcal{K}\right]=\mathcal{K}^{*}(x, y)$. It follows that the three lines in Figure 7 are tangent at $x=x^{*}$.

The threshold $x^{o}$ that solves condition (5.9) is derived by considering the expected return on postponing investment for an infinitesimally short time interval $\mathrm{d} s .^{20}$ If the expected return on an infinitesimally small delay is positive at the point $(x, y)$, then the investor should wait (i.e. $(x, y) \in \mathcal{C}$ ). This enables her to tailor the investment $\mathcal{K}$ to new information regarding the state variables $(x, y)$ that comes to light during the interval $\mathrm{d} s$. However, the trade-off is that she forgoes the net cash flow that would otherwise have been obtained during this short time interval $\mathrm{d} s$. The additional value of the option $V(x, y)$ on top of the net value of the project at optimal investment $(1-\alpha) B^{*}(x, y)$ is that $V(x, y)$ includes the option to postpone investment even further after the time interval $\mathrm{d} s$. Hence, $x^{*} \leq x^{o}$.

Tables 1 and 2 provide critical growth rates and the share of cities that should not invest in real estate due to high growth for various values of $\alpha$ and $\sigma^{2}$. For ease of interpretation, we use the annual growth rates $g^{*}=\theta\left(x^{*}+\mu+\sigma^{2}\right)$ rather than $x$; see equation (2.2). The cell in bold (in the center of the table) corresponds to our benchmark parameters. The critical growth rate $g^{*}$ (above which investment should be delayed) can be quite low: less than $3 \%$ for higher values of $\alpha$ and $\sigma^{2}$. It follows that up to $20 \%$ of cities should optimally delay further construction. These numbers show that stalling new construction and waiting for the market to 'settle' is a realistic possibility affecting a substantial number of cities.

\footnotetext{
${ }^{20}$ The expected return on postponing during $\mathrm{d} s$ is $(1-\alpha)(\mathcal{L}-\rho) B^{*}(x, y) \mathrm{d} s$.
} 
Table 1: Cobb-Douglas critical growth rates $g^{*}=\theta\left(x^{*}+\mu+\sigma^{2}\right)$.

\begin{tabular}{c|ccccc}
\hline & $\sigma^{2}=0.24$ & 0.28 & 0.32 & 0.36 & 0.40 \\
\hline$\alpha=0.25$ & $6.65 \%$ & $6.20 \%$ & $5.72 \%$ & $5.21 \%$ & $4.64 \%$ \\
0.30 & $5.27 \%$ & $4.76 \%$ & $\mathbf{4 . 2 0 \%}$ & $3.57 \%$ & $2.82 \%$ \\
0.35 & $3.97 \%$ & $3.36 \%$ & $2.62 \%$ & $1.65 \%$ & $0.00 \%$ \\
\hline
\end{tabular}

Table 2: Share of cities where investment should be postponed.

\begin{tabular}{c|ccccc}
\hline & $\sigma^{2}=0.24$ & 0.28 & 0.32 & 0.36 & 0.40 \\
\hline$\alpha=0.25$ & $0.1 \%$ & $0.3 \%$ & $0.9 \%$ & $2.4 \%$ & $5.2 \%$ \\
0.30 & $0.7 \%$ & $2.2 \%$ & $\mathbf{5 . 5 \%}$ & $11.3 \%$ & $20.8 \%$ \\
0.35 & $4.3 \%$ & $10.4 \%$ & $20.9 \%$ & $38.0 \%$ & $68.9 \%$ \\
\hline
\end{tabular}

Proposition 8 depends crucially on the form of the production function. The unit elasticity of substitution between construction and land in the Cobb-Douglas production function implies that the investment in construction is a fixed share of the asset value at the moment the option is exercised. Since both cost and revenue are proportional to the cash flow parameter $y$, its level is irrelevant for the moment of investment. This result no longer holds as soon as the elasticity of substitution between both inputs is less than one, as is the case with a production function of the Stone-Geary type, which is considered in the next subsections.

\section{$5.3{ }^{*}$ Stone-Geary: numerical aspects}

This subsection shows that the optimal stopping problem in the Stone-Geary case can be solved by the same method as we used for the Leontief case. In analogy with equation (4.4), we write the Bellman equation satisfied by the option value $V_{\lambda}(x, y)$ as

$$
\begin{aligned}
(\rho-\mathcal{L}) V_{\lambda}(x, y) & =\lambda W_{\lambda}^{-}(x, y) \\
W_{\lambda}(x, y) & :=V_{\lambda}(x, y)-(1-\alpha) B^{*}(x, y)+\rho^{1 /(\alpha-1)},
\end{aligned}
$$

where the value of waiting $W_{\lambda}(x, y)$ is defined in analogy with (4.5). The following proposition presents the Bellman equation for the value of waiting $W_{\lambda}(x, y)$, along with an algorithm for solving the discretized problem in the Stone-Geary case.

Proposition 9 In the Stone-Geary case, the value of waiting $W_{\lambda}(x, y)$ satisfies the Bellman equation

$$
(\rho+\lambda-\mathcal{L}) W_{\lambda}(x, y)=d(x, y)+\lambda W_{\lambda}^{+}(x, y)
$$

where $d(x, y)$, the expected return of a small delay, is

$$
d(x, y):=\rho^{\alpha /(1-\alpha)}-\phi y[\alpha \phi y a(x)]^{\alpha /(1-\alpha)}\left[1-\rho \alpha a(x)-\frac{\sigma^{2}}{2} \frac{\alpha}{1-\alpha} \frac{a^{\prime}(x)^{2}}{a(x)}\right] .
$$


The recursively defined sequence of functions $W_{\lambda}^{(n)}$ as defined in (4.14), but with $1-y$ replaced by $d(x, y)$, is non-decreasing and (at least pointwise) convergent (as $n \rightarrow \infty$ ) to the solution $W_{\lambda}$. For an appropriate discretization $\mathbf{L}$, the recursively defined vector sequence $\underline{W}_{\lambda}^{(n)}$ defined in (4.16), but with $\underline{1}-y$ replaced by $\operatorname{Vec}(\mathbf{D})$, where $\mathbf{D}$ is the (matrix) discretization of $d(x, y)$, is non-decreasing and convergent (as $n \rightarrow \infty$ ) to the limiting vector $\underline{W}_{\lambda}$.

Proposition 9 is a generalization of Propositions 6 and 7. Note that in the Leontief case $(\alpha=0)$, the expected return of a small delay simplifies to $d(x, y)=1-y$. For the StoneGeary case $(\alpha>0)$, unfortunately, no simplification occurs; thus, the functions $a(x)$ and $a^{\prime}(x)$ appearing in $d(x, y)$ must be computed. This can, of course, be done by computing a numerical integral (using standard software) or by using our approximation in Remark 2. The same discretization $\mathbf{L}$ as in Proposition 7 can be used (the construction of $\mathbf{L}$ is discussed in Appendix A.10).

We note that Proposition 9 is more widely applicable to multidimensional optimal stopping problems. It generalizes to other stochastic processes, corresponding to different generators $\mathcal{L}$, and other pay-off functions, which affect the algorithm through the definition of the expected return on a small delay, defined as $d(x, y):=(\mathcal{L}-\rho)[(1-$ $\left.\alpha) B^{*}(x, y)-\rho^{1 /(\alpha-1)}\right]$, which depends both on the stochastic process (through $\mathcal{L}$ ) and the pay-off structure (in square brackets).

\subsection{The investment boundary $y(x)$ for Stone-Geary}

In the Leontief case (see Section 4), the stopping region $\mathcal{S}$ was found to be a subset of the space delineated by two necessary conditions that must hold on the stopping boundary: (i) $y(x) \geq 1$, the cash flow must exceed the interest payment on the investment, and (ii) $\rho A(x, y(x)) \geq 1$, the value of the project must exceed that of the investment; see equations (4.8) and (4.12). The equivalent conditions for the Stone-Geary case are as follows:

$$
\begin{aligned}
(1-\alpha)(\rho-\mathcal{L}) B^{*}(x, y) & \geq \rho^{\alpha /(\alpha-1)}, & (x, y) \in \partial \mathcal{S} \\
(1-\alpha) \rho B^{*}(x, y) & \geq \rho^{\alpha /(\alpha-1)}, & (x, y) \in \partial \mathcal{S} .
\end{aligned}
$$

The first condition states that a share $1-\alpha$ of the return on the asset value must exceed the interest payment on the fixed investment, where the share $\alpha$ is used to pay for the interest on the variable investment. ${ }^{21}$ This condition is equivalent to requiring that $d(x, y) \leq 0$ for $(x, y) \in \partial \mathcal{S}$, where $d(x, y)$ was defined in (5.12). Solving for $y(x)$ (see Appendix A.15)

\footnotetext{
${ }^{21}$ To see the equivalence with condition (4.8), $y \geq 1$, note that the Bellman equation (3.2) implies $(\rho-\mathcal{L}) A(x, y)=y$.
} 
gives rise to the inequality

$$
y(x) \geq \frac{\rho^{\alpha}(1-\alpha)^{1-\alpha}}{a(x)^{\alpha}\left[1-\rho \alpha a(x)-\frac{\sigma^{2}}{2} \frac{\alpha}{1-\alpha} \frac{a^{\prime}(x)^{2}}{a(x)}\right]^{1-\alpha}} \geq 1, \forall x<x^{o},
$$

which holds for $x<x^{o}$, because the right-hand side has a singularity at $x^{o}$. This has an important economic consequence: in both the Cobb-Douglas and the Stone-Geary cases it is never optimal to invest for $x \geq x^{o}$ (i.e. for particularly high growth).

The second condition in (5.13) states that a share $1-\alpha$ of the present value of the net cash flow must exceed the value of the fixed investment, which is $\rho^{1 /(\alpha-1)}$. These considerations justify the following conjecture.

Conjecture 2 The investment boundary y $(x)$ for Stone-Geary satisfies

$$
\begin{array}{ccc}
\mathbf{1} & : & y^{\prime \prime}(x)>0 \\
\mathbf{2} & : & y(x)=\varnothing \text { for } x>x^{*} ; \\
\mathbf{3} & : & \lim _{x \rightarrow x^{*}} A[x, y(x)]=\lim _{x \rightarrow x^{*}} a(x) y(x)=\infty ; \\
\mathbf{4} & : & \lim _{x \rightarrow-\infty} A[x, y(x)]=\lim _{x \rightarrow-\infty} \alpha^{\alpha} \frac{(1-\alpha) \rho y(x)}{\psi-\sigma^{2}-x}=1 ; \\
\mathbf{5} & : & \frac{\mathrm{d} A[x, y(x)]}{\mathrm{d} x}>0
\end{array}
$$

where $x^{*}$ is defined in Proposition 8.

This conjecture is analogous to Conjecture 1. Part one is motivated by the global convexity of the right-hand side of (5.14). This implies that $y^{\prime \prime}(x)$ must be positive at least for some $x$. Part two indicates that it is never optimal to invest for any growth rate $x>x^{*}$, where $x^{*}$ is the same critical threshold as for the Cobb-Douglas case. The reason for this is that $y(x)$ is known to converge to infinity for some threshold value $x^{*} \leq x^{o}$. For that value, the fixed investment is nearly irrelevant relative to $\mathcal{K}^{*}(x, y)$, which diverges to infinity, and the model therefore converges to the Cobb-Douglas case. Part three suggests that in that case the asset value $A[x, y(x)]$ must be infinite since $\lim _{x \rightarrow x^{*}} y(x)=\infty$. Part four is motivated by the second equation of (5.14) and Proposition 2, part three. Part five generalizes the ideas in parts three and four. Although we provide no formal proof for Conjecture 2 beyond the arguments given above, all our numerical results support it (as was the case with Conjecture 1).

Figure 8 shows the function $y(x)$, the conditions (5.13), and the thresholds $x^{*}$ and $x^{o}$. The stopping region $\mathcal{S}$ has an asymptote at $x^{*}$, while the condition $(1-\alpha)(\mathcal{L}-\rho) B^{*}[x, y(x)]=$ $\rho^{\alpha /(1-\alpha)}$ has an asymptote at $x^{o}$. The boundary $y(x)$ of the stopping zone reaches a minimum for $x<\mathrm{E} x$. For higher values of $x$ - that is, for more than $50 \%$ of cities - the curve $y(x)$ slopes upward. This holds a fortiori for the distribution of $x$ conditional on the moment of construction (i.e. when the process $(x, y)$ crosses the boundary $y(x))$. This 


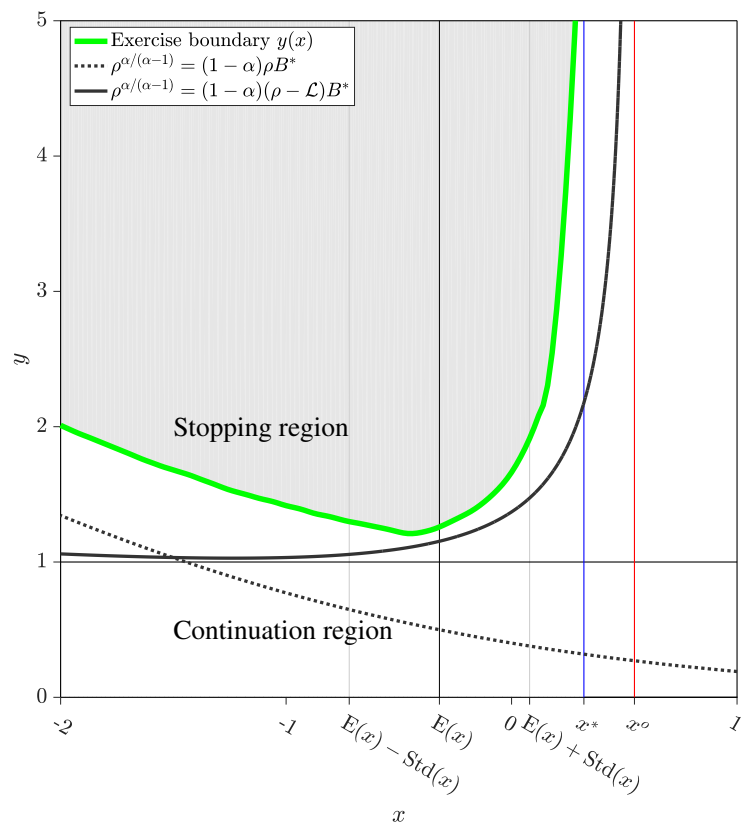

Figure 8: The functions $y(x), \quad(1-\alpha)(\rho-\mathcal{L}) B^{*}[x, y(x)]=\rho^{\alpha /(\alpha-1)}$ and $(1-\alpha) \rho B^{*}[x, y(x)]=\rho^{\alpha /(\alpha-1)}$.

is much more likely to happen for positive than for negative values of $x$, since it requires $y$ to move up (keeping $x$ fixed). The upward-sloping part of the trajectory of $y(x)$ is therefore not a remote possibility that occurs in extreme corners of the state space; it is relevant for more than $50 \%$ of new construction.

Figure 9 shows the function $y(x)$ for a range of values of $\alpha$. The smaller $\alpha$, the more the function looks like the Leontief case discussed in Section 5. However, the upwardsloping part is relevant for a value of $\alpha$ as low as 0.15 ; in that case, if $x$ is two standard deviations above the mean of the steady state distribution, $y(x)$ is approximately $20 \%$ higher than its minimum value.

Figure 10 shows the optimal level of variable investment at the moment of investment as a function of the growth rate $x$. The optimal level of construction varies strongly, in particular for higher values of $\alpha$.

Figure 11 shows the option value in the direction of $y$ for different values of the growth rate $x$. In stark contrast with Figure 4, we see that the option is exercised for high $y$ if the growth rate is low or high, whereas the lowest $y$ required to invest is found for moderate growth rates. Note that the dotted lines represent the value of (optimal) investment, i.e. $(1-\alpha) B^{*}(x, y)-\rho^{1 /(\alpha-1)} \propto y^{1 /(1-\alpha)}$, which is non-linear as a function of $y$. 


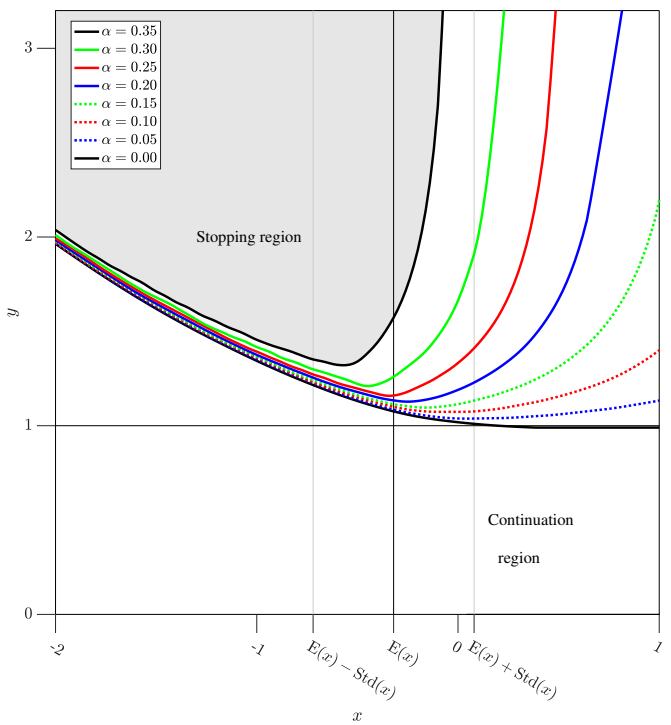

Figure 9: The function $y(x)$ for various values of $\alpha$. The solid green line is the same as in Figure 8.

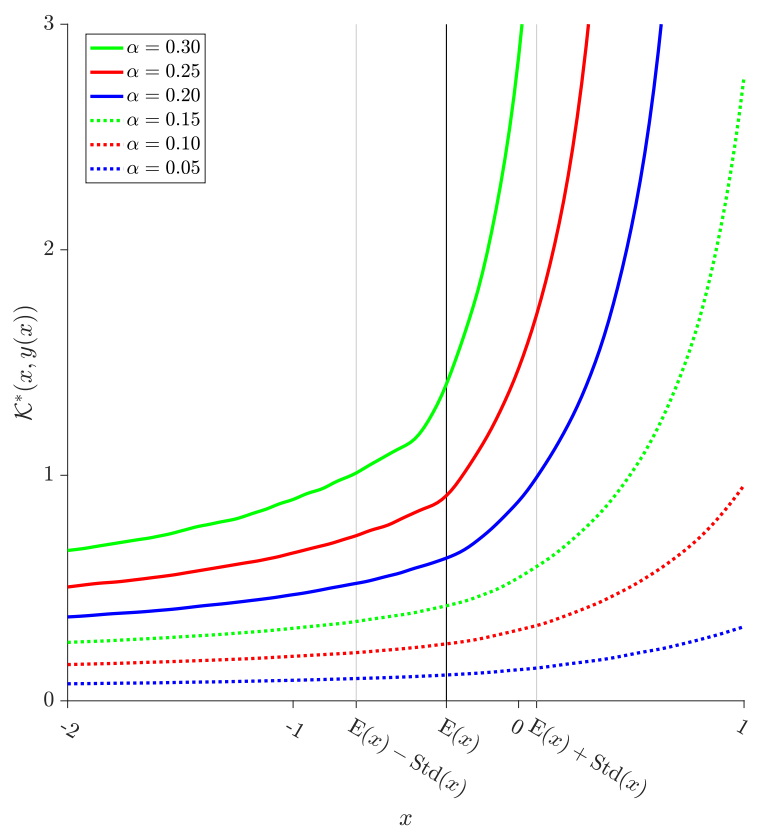

Figure 10: Optimal variable investment at the moment of investment $\mathcal{K}^{*}[x, y(x)]$ for various values of $\alpha$. 


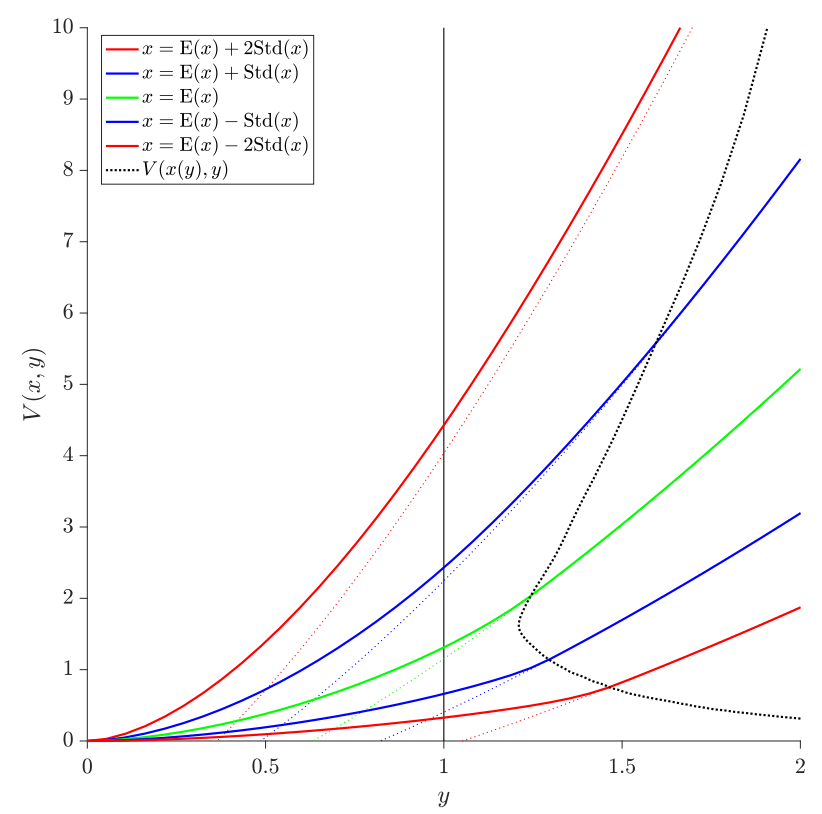

Figure 11: Option value $V(x, y)$ for various levels of $x$.

\section{A city-level model}

\subsection{The Leontief case}

Interpreting the model in the context of cities requires us to flesh out the model from the previous section in a way similar to Capozza and Li (1994), Lucas and Rossi-Hansberg (2002), and Rossi-Hansberg and Wright (2007). People who live in a city can use a public good located in the city center, which is assumed to be concentrated in a 'point' such that it does not take up any space. This is similar to the Central Business District (CBD) in the model of Rossi-Hansberg and Wright (2007). Let $y_{0}$ denote the cash flow of a house (i.e. its rental income) in the CBD. This cash flow is determined by the attractiveness of the CBD. People who live at a distance $d>0$ from the CBD travel from their homes to the city center to enjoy the benefits of the CBD. Suppose that travel cost is determined by a standard iceberg technology, such that the cash flow $y_{d}$ generated by a house at a distance $d$ from the CBD is

$$
y_{d}=y_{0} \mathrm{e}^{-\delta d}
$$

A fixed share of the cash flow is lost for every additional unit of distance of travelling, as in Lucas and Rossi-Hansberg (2002). All results from the previous section continue to apply, except that all functions must be indexed by the distance $d$ to the CBD and the cash flow $y$ must be replaced by the location-specific term $y_{0} \mathrm{e}^{-\delta d}$. Let $D$ be the distance from the edge of the city to the CBD. Locations outside the city, $d>D$, are vacant. We assume that land has no alternative use beyond its transformation into residential 


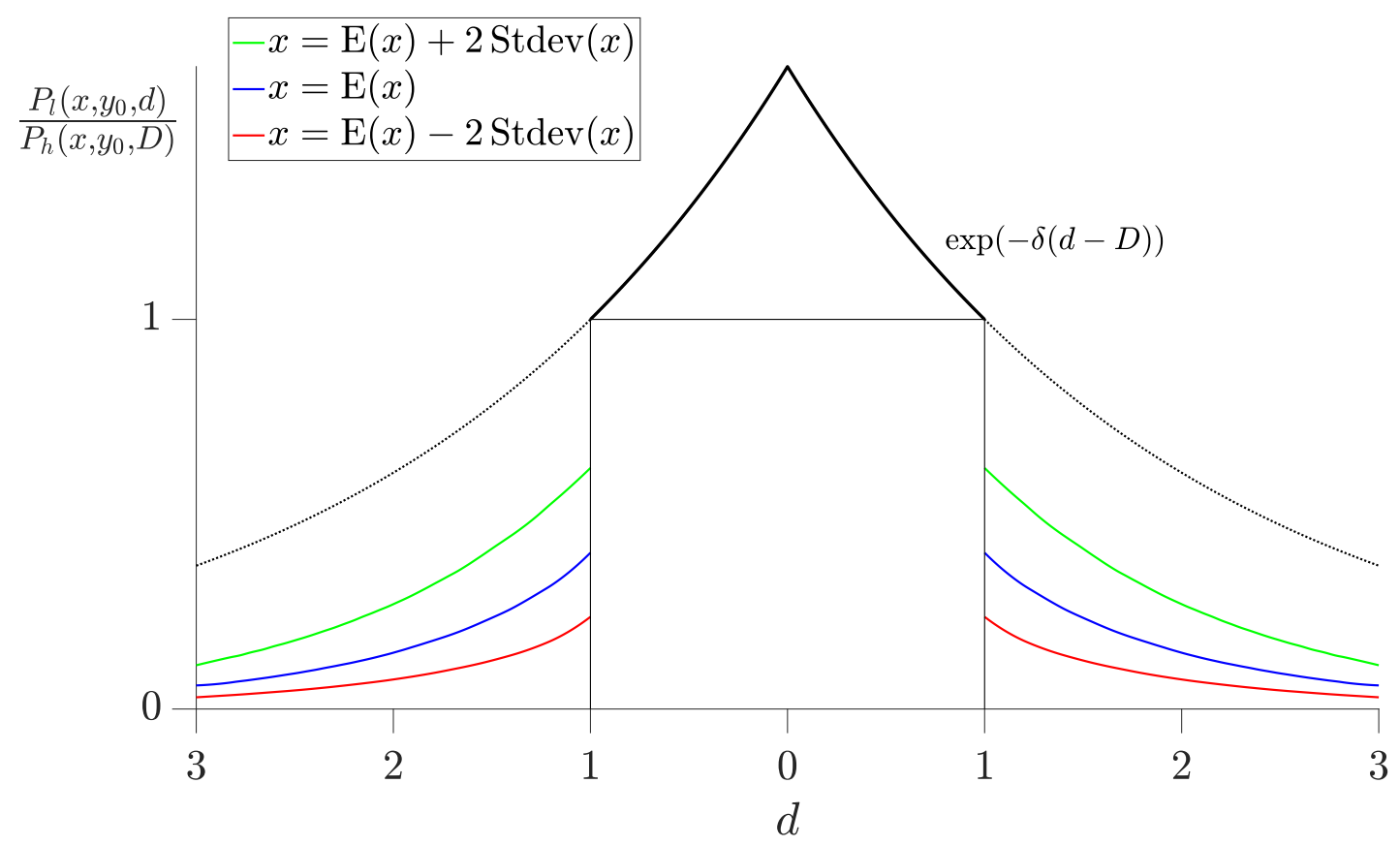

Figure 12: Leontief case for a city with $D=1, \delta=1 / 2$ and various growth rates $x$.

use. If land does have an alternative use, then the fixed investment can be thought of as compensation for the value of the alternative use.

Figure 12 illustrates the situation of a city that is currently constructing new houses. The distance to the city center $d$ is plotted on the horizontal axis, the house/land price on the vertical axis. The house price $P_{h}\left(x, y_{0}, d\right)$ is equal to the asset value of the project as defined in equation (3.1) and (3.3):

$$
P_{h}\left(x, y_{0}, d\right)=A\left(x, y_{0} \mathrm{e}^{-\delta d}\right)=a(x) y_{0} \mathrm{e}^{-\delta d}
$$

In the $\operatorname{CBD}(d=0)$, the house price is $P_{h}\left(x, y_{0}, 0\right)=a(x) y_{0}$, as in Section 3. Moving away from the center, the house price declines exponentially at a rate $\delta$, until we reach the edge of the city at $D$. Since the city is currently constructing new houses, the cash flow net of commuting cost at the edge of the city has to be equal to the construction threshold, i.e.

$$
y_{0} \mathrm{e}^{-\delta D}=y(x)
$$

where $y(x)$ is the exercise curve of the option to build as defined in Section 4. Hence, $P_{h}\left(x, y_{0}, D\right)=a(x) y(x)$.

The bold black curve in Figure 12 is the ratio of the house price at location $d$ relative to that at the edge $D$ of the city

$$
\frac{P_{h}\left(x, y_{0}, d\right)}{P_{h}\left(x, y_{0}, D\right)}=\frac{A\left(x, y_{0} \mathrm{e}^{-\delta d}\right)}{A(x, y(x))}=\mathrm{e}^{-\delta(d-D)},
$$


where we use equation (3.1) in the second step. This ratio does not depend on $x$. For $d \geq D$, land is still vacant. Hence, the function for the price of a house is dotted for these (vacant) locations. The price of vacant land $P_{l}\left(x, y_{0}, d\right)$ outside the city (i.e. $d \geq D$ ) satisfies

$$
P_{l}\left(x, y_{0}, d\right)=V\left(x, y_{0} \mathrm{e}^{-\delta d}\right) \geq a(x) y(x) \mathrm{e}^{-\delta(d-D)}-\rho^{-1},
$$

where equality applies only at the edge of the city, for $d=D$, because the value-matching condition implies that the value of an vacant plot of land equals the value of a house $a(x)$ $y(x)$ minus the construction cost $\rho^{-1}$ at the moment of construction. Outside the city, a vacant plot of land is more valuable than the value of a house minus the construction cost. The further away from the edge of the city, the higher the difference between the price of a vacant plot of land and the value of a house minus its construction cost; the difference is equal to the value of waiting. The ratio

$$
\frac{P_{l}\left(x, y_{0}, D\right)}{P_{h}\left(x, y_{0}, D\right)}=\frac{a(x) y(x)-\rho^{-1}}{a(x) y(x)}
$$

is the land share in the value of a newly constructed house on the edge of the city. The land share is an increasing function of the growth rate $x$. Figure 12 also depicts the value of vacant land beyond the edge of the city, $P_{l}\left(x, y_{0}, d\right) / P_{h}\left(x, y_{0}, D\right)$, for three different levels of $x$ : $\mathrm{E} x$ and $\mathrm{E} x \pm 2 \mathrm{Std} x$. The land share at the edge of a city is $61 \%$ for $x=\mathrm{E} x+2 \mathrm{Std} x$, $41 \%$ for $x=\mathrm{E} x$, and $25 \%$ for $x=\mathrm{E} x-2 \mathrm{Std} x$; see Figure 12. The higher $x$, the smaller the relative decline in the value of land for locations further away from the edge of the city. The reason is that for high growth rates $x$, construction may commence shortly; even on vacant plots located some distance away from the city edge.

In a standard model of cities without a persistent growth rate, the probability that a city is currently transforming vacant land into suburbs at time $s$ is zero. This is due to the excess volatility of a random walk on an infinitesimal interval ds. Any movement of the potential cash flow at the edge of the city, $y_{0} \mathrm{e}^{-\delta D}$, above the construction threshold $y(x)$ is immediately offset by new construction. The equality $y_{0} \mathrm{e}^{-\delta D}=y(x)$ is immediately restored by an increase of $D$. This correction occurs in an infinitesimally short time interval. Afterwards, as soon as a negative shock arrives, $y_{0} \mathrm{e}^{-\delta D}$ drops below the construction threshold, at which point it takes a strictly positive amount of time to restore it. Hence, intervals at the construction threshold are infinitesimal, while intervals below the threshold take a strictly positive amount of time (and therefore $\operatorname{Pr}\left[y_{0} \mathrm{e}^{-\delta D}<y(x)\right]=1$ ). ${ }^{22}$ This is not the case for our model, where the growth follows an Ornstein-Uhlenbeck process. In our case, $y_{s}$ is differentiable with respect to $s$. If the growth is positive, this rate will remain positive for the next short time interval $\mathrm{d} s$. In the steady state, the

\footnotetext{
${ }^{22}$ This can be proven by applying the reflection principle, which allows us to calculate the probability distribution of $y_{0} \mathrm{e}^{-\delta D}$, which has no mass point at a reflecting boundary.
} 
probability that a city has a positive growth rate is

$$
\operatorname{Pr}\left[x+\mu+\sigma^{2}>0\right]=\Phi\left(\frac{\sqrt{2} \mu}{\sigma}\right),
$$

where $\Phi(\cdot)$ is the cumulative distribution function of a standard normal distribution; see equations $(2.2)$ and $(2.7) \cdot{ }^{23}$

The model developed in this section implies a relation between growth rates of the cash flow $y_{d}$ and the total population of the city. We assume the land use per person is fixed; without loss of generality, it is normalized to unity. Then, the population of a city, $N$, equals the surface of a circle with radius $D$, the distance from the CBD until the edge of the city; in logs

$$
\ln N=\ln \pi+2 \ln D .
$$

This relation between $\ln N$ and $\ln D$ is linear; both series therefore have the same degree of persistence in an $\mathrm{AR}(1)$ regression. Substitution of equation (6.1) in equation (6.2) yields:

$$
\ln y_{d}=\ln y(x)+\delta(D-d) .
$$

For a city with a constant growth rate $x$, which is currently constructing new housing on its edge, the $\log$ cash flow generated by a house at a particular location $d, \ln y_{d}$, grows linearly with the radius $D$ of the city. Even as $x$ varies over time, $y(x)$ remains fairly constant as long as $x>0$, see Figure 3. Hence the persistence of the population growth of expanding cities in this Leontieff model reasonably approximates the persistence of the cash flow growth in those cities. ${ }^{24}$ For $x<0$ this argument fails, but in any event shrinking cities are unlikely to be constructing new housing. This is consistent with the argument by Glaeser and Gyourko (2005) that house prices in shrinking cities are more responsive to economic fluctuations, while construction is more responsive in growing cities.

\subsection{Extension to the Stone-Geary case}

In the more general Stone-Geary case, the density of construction varies with the growth rate at the moment of construction; see Section 6. The price of a house and the price of

\footnotetext{
${ }^{23}$ Positive growth is not a sufficient condition for a city to be extending its built area, since $x+\mu+\sigma^{2}>0$ does not necessarily imply that $y_{0} \mathrm{e}^{-\delta D}=y(x)$. A growing city might be larger than is optimal from a current investor's perspective, since $y_{0}$ has shrunk in the past. Since construction is irreversible, a city must first grow for some time before it hits the construction threshold again.

${ }^{24}$ Equality in the persistence of the growth in $\ln N$ and $\ln y_{d}$ holds exactly for $x>0$ if we replace the exponential law in (6.1) by the power law $y_{d}=y_{1} d^{-\delta}$ for $\delta>0$. A power law fits the data well; see e.g. Figures 2.1 and 2.2 in Glaeser (2008). However, it implies the price of land at $d=0$ is infinite; hence the cash flow is normalised at $d=1$. We can interpret this model if the CBD covers the area $d<1$, while commuting costs are relative to the edge of the CBD located at $d=1$.
} 
vacant land therefore satisfy

$$
\begin{aligned}
P_{h}\left(x, y_{0}, d, \mathcal{K}\right) & =\phi \mathcal{K}^{\alpha} A\left(x, y_{0} \mathrm{e}^{-\delta d}\right) \\
P_{l}\left(x, y_{0}, d\right) & =V\left(x, y_{0} \mathrm{e}^{-\delta d}\right)
\end{aligned}
$$

By equation (3.1), $\ln P_{h}\left(x, y_{0}, d\right)$ satisfies the $\log$ linear relation

$$
\ln P_{h}\left(x, y_{0}, d, \mathcal{K}\right)=\ln \phi+\alpha \ln \mathcal{K}+\ln a(x)+\ln y_{0}-\delta d
$$

For large $x, \ln a(x) \approx$ constant $+\left[a^{\prime}(x) / a(x)\right] x \approx$ constant $+x$. This follows by Proposition 2 , which implies $\lim _{x \rightarrow \infty}\left[a^{\prime}(x) / a(x)\right]=1$. The unit coefficient on the growth rate $x$ for the limiting case $\lim x \rightarrow \infty$ is due to the application of our transformed time scale $s=\theta t$. The advantage of this equation is that it can be estimated.

The relation between the log house price and the density of construction is determined by an additive term $\alpha \ln \mathcal{K}$. Within the city, the density of existing buildings varies only with the historical growth rate of the city, denoted by $\bar{x}$, prevalent when these buildings were constructed. If the decision to build was optimal, then the historical cash flow in the $\mathrm{CBD}$, denoted by $\bar{y}_{0}$, and the historical growth rate $\bar{x}$ are related through the relation $y(\bar{x})=\bar{y}_{0} \mathrm{e}^{-\delta d}$, where $y(x)$ is the critical curve for the Stone-Geary investment decision as defined in section 5. If, moreover, the density of historical construction, denoted by $\overline{\mathcal{K}}$, was optimal, then

$$
\overline{\mathcal{K}}=\mathcal{K}^{*}\left[\bar{x}, \bar{y}_{0} \mathrm{e}^{-\delta d}\right]=\mathcal{K}^{*}[\bar{x}, y(\bar{x})]
$$

Thus $\overline{\mathcal{K}}=\overline{\mathcal{K}}(\bar{x})$. Moreover, this function is one-to-one. The growth rate $\bar{x}$ that applied at the time that various locations $d$ were added to the city varies across locations within the city; hence, $\bar{x}$ is a function of this location index $d, \bar{x}(d)$. This variation in historical growth rates $\bar{x}$ and corresponding densities $\overline{\mathcal{K}}$ across the city implies that equation (6.4) can be estimated in practice. $^{25}$

The Stone-Geary production technology implies that the marginal value of construction satisfies

$$
\frac{\mathrm{d} P_{h}\left(x, y_{0}, d, \mathcal{K}\right)}{\mathrm{d} \mathcal{K}}=\alpha \frac{P_{h}\left(x, y_{0}, d, \mathcal{K}\right)}{\mathcal{K}} .
$$

Other things (i.e.: $x, y$, and $d$ ) being equal, the value of the marginal unit of construction declines as a function of its density: $\mathrm{d}^{2} P_{h}\left(x, y_{0}, d, \mathcal{K}\right) /(\mathrm{d} \mathcal{K})^{2}<0$. At the edge of a growing city, the value of the marginal unit of construction $\mathcal{K}$ must be equal to its construction

\footnotetext{
${ }^{25}$ In a richer, more realistic model, one would also allow for the demolition of old, low-density buildings or buildings with different purposes (e.g. industrial buildings) in order to replace them with new construction, the density of which is optimally adjusted to the current growth rate $x$ and the current cash flow net of commuting cost $y_{0} \mathrm{e}^{-\delta d}$. This would provide an additional source of variation in observed densities $\overline{\mathcal{K}}$, thereby further facilitating the estimation of (6.4).
} 
cost,

$$
\frac{\mathrm{d} P_{h}\left(x, y_{0}, D, \mathcal{K}\right)}{\mathrm{d} \mathcal{K}}=\alpha \frac{P_{h}\left(x, y_{0}, D, \mathcal{K}\right)}{\mathcal{K}}=1
$$

The share of (fixed and variable) construction in the house price evaluated at the marginal price of construction is

$$
\text { construction share }=\frac{\mathcal{K}+\rho^{-1}}{P_{h}\left(x, y_{0}, D, \mathcal{K}\right)}=\alpha \frac{\rho \mathcal{K}+1}{\rho \mathcal{K}} .
$$

The share of construction for newly constructed buildings therefore decreases with the variable investment $\mathcal{K}$, and for high densities $\mathcal{K}$ converges to the level $\alpha$. The declining cost share is due to the minimum necessary investment of $\rho^{-1}$ per unit of land or, equivalently, the fact that the elasticity of substitution between land and construction in the production of housing services is smaller than unity. If a city continues growing after the moment of construction at location $d$, then the current marginal value of construction at that location exceeds the cost of construction. The reverse holds when a city has been shrinking since the moment of construction.

Let $P_{l}\left(x, y_{0}, d\right)$ denote the price of vacant land as a function of $x, y_{0}$ and $d$ (for $d>D$, since all land located within the city, $d \leq D$, is already transformed into built area). Assume the city is extending on its edge, such that $y_{0} \mathrm{e}^{-\delta D}=y(x)$. It follows from equation (5.5) that the price of vacant land at the moment of construction is equal to

$$
P_{l}\left(x, y_{0}, D\right)=(1-\alpha) \widetilde{\alpha} A[x, y(x)]^{1 /(1-\alpha)}-\rho^{-1}
$$

Hence, the price elasticity of land with respect to the city's growth rate is

$$
\frac{\mathrm{d} \ln P_{l}\left(x, y_{0}, D\right)}{\mathrm{d} x}=\frac{\widetilde{\alpha} A(x, y)^{1 /(1-\alpha)}}{(1-\alpha) \widetilde{\alpha} A(x, y)^{1 /(1-\alpha)}-\rho^{-1}}\left[\frac{a^{\prime}(x)}{a(x)}+\frac{y^{\prime}(x)}{y(x)}\right]
$$

This expression exceeds the direct effect $a^{\prime}(x) / a(x)$ for relevant values of $x$ for two reasons. First, the ratio before the term in square brackets is larger than one. For a Cobb-Douglas technology, the value of construction is a fixed share of the value of a house. For a Stone-Geary technology, the share of construction is a declining function of the house price due to the declining share of the fixed investment. An increase in the growth rate therefore increases the value of vacant land more than proportionally. Second, the relevant part of the exercise boundary $y(x)$ is upward sloping (see Figure 9; the relevant part is $x>\mathrm{E} x$, since there is hardly any construction for $x \leq \mathrm{E} x$ ). The elasticity for vacant land is therefore greater than the elasticity for constructed houses (which is $a^{\prime}(x) / a(x)$ ). Hence an increase in the growth rate $x$ has a larger effect on the the price of vacant land than on the prices of existing houses in the city.

This conclusion has important policy implications. Glaeser and Gyourko (2002) have argued that high prices of vacant land point to the presence of regulatory constraints 
with regard to city extension. We show that the price of vacant land on the edge of a city is particularly sensitive to the city's growth rate. Our model contains no market distortions; hence, from the price of vacant land alone, we cannot draw immediate conclusions regarding the efficiency of the transformation of agricultural land into suburbs. In fact, city planners and real estate developers may be acting rationally in leaving valuable vacant land untouched for some time before commencing construction.

\section{Conclusion}

One would expect the optimal moment to exercise the option to build on vacant land to be described by a trade-off between the level of the cash flow and its growth: commence construction when either the cash flow or its growth is high. We show that for a practically relevant range of positive growth rates of the cash flow, the slope of this trade-off runs counter to this simple intuition. The reason for this counterintuitive result is twofold. First, due to the irreversibility of construction, when the growth rate is high the investor chooses the density of construction, keeping in mind the expected future increased cash flow. She therefore chooses a density that is higher than justified by the current cash flow. This makes the project less attractive under current market conditions. The current rental income might not even cover the interest on the investment. In this case, delaying the investment is the optimal response. This argument becomes all the more pertinent at high growth rates. Second, during the period of delay, new information on the future evolution of the growth rate comes to light, which can be used to better tailor the density of construction to its future use. The higher the growth, the greater the value of this additional information. Our numerical results show that this finding is by no means a rare phenomenon relevant only in the extreme right tail of the distribution of the city's growth rates; rather, it applies to all growing cities and hence all new construction.

These results were obtained under the assumption that the growth of the cash flow follows an Ornstein-Uhlenbeck process. Although the asset value of a project with this characteristic has no closed-form solution, we provide convenient and accurate approximations using the standard Gamma function. We expect this formulation to have many applications outside the realm of cities; for example, in the valuation of the equity of high-growth companies. The model gives a more realistic representation of cash flows than the standard random-walk cum drift assumption, which implies, unrealistically, that the current growth rate has no predictive power for future growth. Extrapolating from current growth rates, one would expect that in the coming decade, Google will grow and the newspaper industry will decline. The available empirical evidence on the growth of cities supports our assumption; see e.g. Albouy et al. (2014) and Nur and Teulings (in prep). Our model provides a natural explanation of why, in the short run, asset values are substantially more volatile than cash flows. It might be interesting to combine these ideas 
with uncertainty shocks as in Bloom (2009); a shock in the level of the growth rate can have a major impact on the level of investment in the short run, leading to an increase in volatility.

The counterintuitive positive relation between the critical level of the cashflow and its growth at the exercise boundary implies that the price of vacant land at the edge of a city is highly sensitive to the city's growth. This calls into question a suggested interpretation in Glaeser and Gyourko (2002): that the high price of land in cities is a tell-tale sign of regulatory constraints. Rather, the higher the city's growth rate, the higher the price of land.

We have analyzed these issues in a simple model of a city where the magnitude of the cash flows is treated as an exogenous process, independent of new construction. In this case there are no agglomeration externalities; hence, private landownership generates an efficient outcome in both the timing and the density of construction. In the more general case where new construction generates agglomeration externalities, private ownership yields inefficiencies; see Rossi-Hansberg (2004). Private investors do not account for the positive externalities that new construction generates for the city's incumbent population. The interaction of these externalities and the option value of postponing investment is a topic for future research.

\section{Acknowledgements}

Our thanks go to David Newbery, Jamil Nur, Daniel Ralph, Jordan Rappaport and Dick van Dijk for their valuable comments. 


\section{References}

M. Abramovich and I. Stegun. Handbook of Mathematical Functions with Formulas, Graphs and Mathematical Tables, volume 55. National Bureau of Standards, 10th edition, 1972.

J. Ahlberg and E. Nilson. Convergence properties of the spline fit. Journal of the Society for Industrial and Applied Mathematics, 11(1):95-104, 1963.

D. Albouy, G. Ehrlich, and Y. Liu. Housing demand and expenditures: How rising rent levels affect behavior and cost-of-living over space and time. In CEP/LSE Labour Seminar, London, volume 28, 2014.

L. H. Alvarez. Solving optimal stopping problems of linear diffusions by applying convolution approximations. Mathematical Methods of Operations Research, 53(1):89-99, 2001.

L. Andersen and M. Broadie. Primal-dual simulation algorithm for pricing multidimensional american options. Management Science, 50(9):1222-1234, 2004.

R. J. Arnott and F. D. Lewis. The transition of land to urban use. Journal of Political Economy, 87(1):161-169, 1979.

V. Bally and J. Printems. A quantization tree method for pricing and hedging multidimensional american options. Mathematical Finance, 15(1):119-168, 2005.

N. Bloom. The impact of uncertainty shocks. Econometrica, 77(3):623-685, 2009.

D. Capozza and Y. Li. The intensity and timing of investment: The case of land. The American Economic Review, pages 889-904, 1994.

D. R. Capozza and R. W. Helsley. The fundamentals of land prices and urban growth. Journal of Urban Economics, 26(3):295-306, 1989.

L. K. Chan, J. Karceski, and J. Lakonishok. The level and persistence of growth rates. The Journal of Finance, 58(2):643-684, 2003.

M. A. Davis and J. Heathcote. The price and quantity of residential land in the united states. Journal of Monetary Economics, 54(8):2595-2620, 2007.

M. A. Davis and M. G. Palumbo. The price of residential land in large us cities. Journal of Urban Economics, 63(1):352-384, 2008.

K. Desmet and J. Rappaport. The settlement of the united states, 1800-2000: The long transition towards gibrat's law. Journal of Urban Economics, 2015. 
Y. d'Halluin, P. A. Forsyth, and G. Labahn. A penalty method for american options with jump diffusion processes. Numerische Mathematik, 97(2):321-352, 2004.

A. K. Dixit and R. S. Pindyck. Investment Under Uncertainty. Princeton University Press, 1994.

P. A. Forsyth and K. R. Vetzal. Quadratic convergence for valuing american options using a penalty method. SIAM Journal on Scientific Computing, 23(6):2095-2122, 2002.

E. L. Glaeser. Cities, Agglomeration, and Spatial Equilibrium. Oxford University Press, 2008.

E. L. Glaeser and J. Gyourko. The impact of zoning on housing affordability. Technical report, National Bureau of Economic Research, 2002.

E. L. Glaeser and J. Gyourko. Urban decline and durable housing. Journal of Political Economy, 113(2):345-375, 2005.

I. Karatzas and S. Shreve. Brownian Motion and Stochastic Calculus, volume 113. Springer Science \& Business Media, New York, NY, 2012.

H. D. Kwon and S. A. Lippman. Acquisition of project-specific assets with bayesian updating. Operations Research, 59(5):1119-1130, 2011.

R.-J. Lange, D. Ralph, and J. van Casteren. Optimal stopping of hunt processes at random poisson times. Manuscript in preparation, in prep.

R. E. Lucas and E. Rossi-Hansberg. On the internal structure of cities. Econometrica, 70 (4):1445-1476, 2002.

J. Nur and C. Teulings. On the persistence of urban growth. Manuscript in preparation, in prep.

B. Øksendal. Stochastic Differential Equations. Springer-Verlag, Heidelberg, Germany, 6th edition, 2007.

G. Peskir and A. Shiryaev. Optimal Stopping and Free-Boundary Problems. Birkhäuser, Basel, Switzerland, 2006.

R. J. Plemmons. M-matrix characterizations. i - nonsingular m-matrices. Linear Algebra and its Applications, 18(2):175-188, 1977.

D. Revuz and M. Yor. Continuous martingales and Brownian motion, vol. 293 of Grundlehren der Mathematischen Wissenschaften. Springer-Verlag, Berlin, 1999. 
L. C. Rogers. Monte carlo valuation of american options. Mathematical Finance, 12(3): 271-286, 2002.

L. C. Rogers and D. Williams. Diffusions, Markov Processes, and Martingales. Volume 1: Foundations. Cambridge University Press., 2000.

L. C. Rogers and O. Zane. A simple model of liquidity effects. In Advances in Finance and Stochastics, pages 161-176. Springer, 2002.

E. Rossi-Hansberg. Optimal urban land use and zoning. Review of Economic Dynamics, $7(1): 69-106,2004$.

E. Rossi-Hansberg and M. L. Wright. Urban structure and growth. The Review of Economic Studies, 74(2):597-624, 2007.

G. E. Uhlenbeck and L. S. Ornstein. On the theory of the brownian motion. Physical Review, 36(5):823, 1930.

K. Zhang, X. Yang, and K. L. Teo. Convergence analysis of a monotonic penalty method for american option pricing. Journal of Mathematical Analysis and Applications, 348 (2):915-926, 2008.

K. Zhang, S. Wang, X. Yang, and K. L. Teo. A power penalty approach to numerical solutions of two-asset american options. Numerical Mathematics: Theory, Methods and Applications, 2:202-223, 2009.

R. Zvan, P. A. Forsyth, and K. R. Vetzal. Penalty methods for american options with stochastic volatility. Journal of Computational and Applied Mathematics, 91(2):199218, 1998. 


\section{A Appendix}

\section{A.1 Proof of Proposition 1}

The pair of SDEs (2.5) can be written jointly in matrix form as

$$
\mathrm{d}\left(\begin{array}{c}
x_{s} \\
\ln y_{s}
\end{array}\right)=\left(\begin{array}{cc}
-1 & 0 \\
1 & 0
\end{array}\right)\left(\begin{array}{c}
x_{s} \\
\ln y_{s}
\end{array}\right) \mathrm{d} s+\left(\begin{array}{c}
-\sigma^{2} \\
\mu+\sigma^{2}
\end{array}\right) \mathrm{d} s+\left(\begin{array}{cc}
\sigma & 0 \\
0 & 0
\end{array}\right)\left(\begin{array}{c}
\mathrm{d} W_{s}^{(1)} \\
\mathrm{d} W_{s}^{(2)}
\end{array}\right) .
$$

Following Karatzas and Shreve (2012) (section 5.6, p. 354), the solution can be written as

$$
\left(\begin{array}{c}
x_{s} \\
\ln y_{s}
\end{array}\right)=M(s)\left[\left(\begin{array}{c}
x_{0} \\
\ln y_{0}
\end{array}\right)+\int_{0}^{s} M^{-1}(z)\left(\begin{array}{c}
-\sigma^{2} \\
\mu+\sigma^{2}
\end{array}\right) \mathrm{d} z+\int_{0}^{s} M^{-1}(z)\left(\begin{array}{cc}
\sigma & 0 \\
0 & 0
\end{array}\right)\left(\begin{array}{c}
\mathrm{d} W_{z}^{(1)} \\
\mathrm{d} W_{z}^{(2)}
\end{array}\right)\right]
$$

where $\mathrm{d} W_{z}^{(i)}$ for $i=1,2$ are the increments of two independent Brownian motions, and the (unknown) $2 \times 2$ matrix $M(s)$ satisfies a first-order differential equation with initial condition as follows

$$
M(0)=\left(\begin{array}{ll}
1 & 0 \\
0 & 1
\end{array}\right), \quad \frac{\mathrm{d}}{\mathrm{d} s} M(s)=\left(\begin{array}{cc}
-1 & 0 \\
1 & 0
\end{array}\right) M(s)
$$

It is straightforward to work out that $M(s)$ and its inverse $M^{-1}(s)$ are given by

$$
M(s)=\left(\begin{array}{cc}
\mathrm{e}^{-s} & 0 \\
1-\mathrm{e}^{-s} & 1
\end{array}\right), \quad M^{-1}(s)=\left(\begin{array}{cc}
\mathrm{e}^{s} & 0 \\
1-\mathrm{e}^{s} & 1
\end{array}\right)
$$

It follows that $\left(x_{s}, \ln y_{s}\right)^{\prime}$ is jointly normally distributed with mean

$$
\begin{aligned}
m_{s} & =M(s)\left[\left(\begin{array}{c}
x_{0} \\
\ln y_{0}
\end{array}\right)+\int_{0}^{s} M^{-1}(z)\left(\begin{array}{c}
-\sigma^{2} \\
\mu+\sigma^{2}
\end{array}\right) \mathrm{d} z,\right] \\
& =\left(\begin{array}{cc}
\mathrm{e}^{-s} & 0 \\
1-\mathrm{e}^{-s} & 1
\end{array}\right)\left[\left(\begin{array}{c}
x_{0} \\
\ln y_{0}
\end{array}\right)+\int_{0}^{s}\left(\begin{array}{cc}
\mathrm{e}^{z} & 0 \\
1-\mathrm{e}^{z} & 1
\end{array}\right)\left(\begin{array}{c}
-\sigma^{2} \\
\mu+\sigma^{2}
\end{array}\right) \mathrm{d} z\right], \\
& =\left(\begin{array}{c}
\sigma^{2}+\left(x_{0}+\sigma^{2}\right) \mathrm{e}^{-s} \\
\ln y_{0}+\mu s+\left(x_{0}+\sigma\right)\left(1-\mathrm{e}^{-s}\right)
\end{array}\right),
\end{aligned}
$$

and covariance matrix

$$
\begin{aligned}
\Sigma_{s} & =M(s)\left[\int_{0}^{s} M^{-1}(z)\left(\begin{array}{ll}
\sigma & 0 \\
0 & 0
\end{array}\right)^{2}\left(M^{-1}(z)\right)^{\prime} \mathrm{d} z\right] M(s)^{\prime}, \\
& =\left(\begin{array}{cc}
\mathrm{e}^{-s} & 0 \\
1-\mathrm{e}^{-s} & 1
\end{array}\right)\left[\int_{0}^{s}\left(\begin{array}{cc}
\mathrm{e}^{z} & 0 \\
1-\mathrm{e}^{z} & 1
\end{array}\right)\left(\begin{array}{cc}
\sigma & 0 \\
0 & 0
\end{array}\right)^{2}\left(\begin{array}{cc}
\mathrm{e}^{z} & 1-\mathrm{e}^{z} \\
0 & 1
\end{array}\right) \mathrm{d} z\right]\left(\begin{array}{cc}
\mathrm{e}^{-s} & 1-\mathrm{e}^{-s} \\
0 & 1 \\
1
\end{array}\right), \\
& =\frac{1}{2} \sigma^{2}\left(\begin{array}{cc}
1-\mathrm{e}^{-2 s} & \left(1-\mathrm{e}^{-s}\right)^{2} \\
\left(1-\mathrm{e}^{-s}\right)^{2} & 2 s-4\left(1-\mathrm{e}^{-s}\right)+\left(1-\mathrm{e}^{-2 s}\right)
\end{array}\right) .
\end{aligned}
$$

The steady-state distribution follows by taking the limit $s \rightarrow \infty$.

\section{A.2 Representation by Gamma function}

For three parameters $a, c \in \mathbb{R}_{>0}$ and $b \in \mathbb{R}$, it holds that

$$
\begin{aligned}
& \int_{0}^{\infty} \exp \left[-a t+b\left(1-e^{-c t}\right)\right] \mathrm{d} t=\frac{e^{b}}{c}\left[b^{-a / c} \gamma(a / c, b)\right], \quad \text { for } b \neq 0, \\
& \int_{0}^{\infty} \exp \left[-a t+b\left(1-e^{-c t}\right)\right] \mathrm{d} t=a^{-1}, \quad \text { for } b=0,
\end{aligned}
$$


where $\gamma(\cdot, \cdot)$ denotes the incomplete lower Euler gamma function. The incomplete lower Euler gamma function is defined as $\gamma(a, b)=\int_{0}^{b} t^{a-1} e^{-t} d t$. For reference, the incomplete upper Euler gamma function is defined by $\Gamma(a, b)=\int_{b}^{\infty} t^{a-1} e^{-t} d t$. The sum of both Euler gamma functions equals the complete Euler gamma function, denoted by $\Gamma(a)=\gamma(a, b)+\Gamma(a, b)=$ $\int_{0}^{\infty} t^{a-1} e^{-t} d t$

To prove our statement above, we define a new time coordinate $\tau=b e^{-c t}$ such that $\tau \in[0, b]$ or $\tau \in[b, 0]$, since $b$ might be negative. Hence we may write $\tau \in[\min (0, b), \max (0, b)]$. Note that if $b<0$, then $\tau<0$, such that $\tau$ has the same sign as $b$. The inverse transformation, that is from $\tau$ to $t$, is given by $t=c^{-1} \ln |b|-c^{-1} \ln |\tau|$, while the differential form is $\mathrm{d} t=-(c \tau)^{-1} \mathrm{~d} \tau$. The integrand then reads

$$
\begin{aligned}
\exp \left[-a t+b\left(1-e^{-c t}\right)\right] \mathrm{d} t & =-(c \tau)^{-1} \exp [-a / c \ln |b|+a / c \ln |\tau|+b-\tau] \mathrm{d} \tau \\
& =-\operatorname{sign}(b) c^{-1}|b|^{-a / c}|\tau|^{a / c-1} e^{b-\tau} \mathrm{d} \tau .
\end{aligned}
$$

where we have used $1 / \tau=\operatorname{sign}(b) /|\tau|$. It follows that the integral can be written as

$$
\begin{aligned}
\int_{0}^{\infty} \exp \left[-a t+b\left(1-e^{-c t}\right)\right] \mathrm{d} t & =-\operatorname{sign}(b) \frac{e^{b}}{c}|b|^{-a / c} \int_{b}^{0}|\tau|^{a / c-1} e^{-\tau} \mathrm{d} \tau \\
& =\operatorname{sign}(b) \frac{e^{b}}{c}|b|^{-a / c} \int_{0}^{b}|\tau|^{a / c-1} e^{-\tau} \mathrm{d} \tau \\
& =\frac{e^{b}}{c}\left[b^{-a / c} \gamma(a / c, b)\right]
\end{aligned}
$$

The final equality follows by the definition of the incomplete lower Euler gamma function above. Note that for $b<0$ in conjunction with $a / c<1$, both $b^{-a / c}$ and $\gamma(a / c, b)$ are imaginary numbers. However, as shown in the penultimate step, their product is a real positive number. Hence the term in square brackets is always real and positive. In computing the term in square brackets, some computer packages will produce a very small imaginary part, which can safely be ignored. Other computer programs simply do not compute the incomplete lower Euler gamma function $\gamma(a / c, b)$ for $b<0$ and produce the output zero in this case. For such packages the penultimate line must be used.

\section{A.3 Proof of Proposition 2}

It is convenient to write equation (3.3) as

$$
\begin{aligned}
a(x) & =\int_{0}^{\infty} e^{g(x, s)} d s, \quad g(x, s):=x\left(1-e^{-s}\right)+\frac{\sigma^{2}}{4}\left(1-e^{-2 s}\right)-\psi s, \quad g(x, 0)=0, \\
a^{\prime}(x) & =\int_{0}^{\infty} g_{x}(x, s) e^{g(x, s)} d s, \quad g_{x}(x, s)=1-e^{-s} \\
a^{\prime \prime}(x) & =\int_{0}^{\infty} \int_{0}^{\infty} g_{x}^{2}(x, s) e^{g(x, s)} d s, \\
g_{s}(x, s) & =x e^{-s}+\frac{\sigma^{2}}{2} e^{-2 s}-\psi, \quad g_{s s}(x, s)=-x e^{-s}-\sigma^{2} e^{-2 s} .
\end{aligned}
$$

We prove each part in turn:

1. By taking $a=\psi, b=\sigma^{2} / 4$ and $c=2$ in equation (A.1), we obtain

$$
a(0)=\frac{1}{2} \exp \left(\frac{\sigma^{2}}{4}\right)\left(\frac{\sigma}{4}\right)^{-\psi / 2} \gamma\left(\frac{\psi}{2}, \frac{\sigma^{2}}{4}\right)>\psi^{-1}
$$

2. This follows immediately from $g_{x}(x, s)=1-e^{-s} \in[0,1)$ for all $s \in \mathbb{R}_{\geq 0}$.

3. The proof is an analogous to Jensen's inequality, which implies that $\mathrm{E}\left[z^{2}\right]>\mathrm{E}[z]^{2}$ for a random variable $z$ with strictly positive variance. In this context, $a(x)^{-1} e^{g(x, s)}$ can be interpreted as a density function of $s$. Hence

$$
\frac{a^{\prime}(x)}{a(x)}=\mathrm{E}\left[g_{x}(x, s)\right], \quad \frac{a^{\prime \prime}(x)}{a(x)}=\mathrm{E}\left[g_{x}(x, s)^{2}\right]
$$

It follows that

$$
\left(\frac{a^{\prime}(x)}{a(x)}\right)^{2}<\frac{a^{\prime \prime}(x)}{a(x)}
$$


4. We decompose the integral in parts

$$
\int_{0}^{\infty} e^{g(x, s)} \mathrm{d} s=\sum_{k=1}^{\infty} \int_{(k-1)|x|^{-2 / 3}}^{k|x|^{-2 / 3}} e^{g(x, s)} \mathrm{d} s
$$

Since for $x<\sigma^{2}, g_{s}(x, s)<0$ and $g_{s s}(x, s)>0, g(x, s)$ satisfies for $s^{-} \leq s \leq s^{+}$

$$
g\left(x, s^{-}\right)+g_{s}\left(x, s^{-}\right)\left(s-s^{-}\right) \leq g(x, s) \leq g\left(x, s^{-}\right)+g_{s}\left(x, s^{+}\right)\left(s-s^{-}\right) .
$$

Integrating the exponential of these three expressions from $s=s^{-}$to $s^{+}$yields

$$
e^{g\left(x, s^{-}\right)} \int_{s^{-}}^{s^{+}} e^{g_{s}\left(x, s^{-}\right)\left(s-s^{-}\right)} \mathrm{d} s \leq \int_{s^{-}}^{s^{+}} e^{g(x, s)} \mathrm{d} s \leq e^{g\left(x, s^{-}\right)} \int_{s^{-}}^{s^{+}} e^{g_{s}\left(x, s^{+}\right)\left(s-s^{-}\right)} \mathrm{d} s .
$$

Evaluating the integrals in the first and third expression and multiplying by $g_{s}\left(x, s^{-}\right)$yields

$$
e^{g\left(x, s^{-}\right)}\left[1-e^{g_{s}\left(x, s^{-}\right)\left(s^{+}-s^{-}\right)}\right] \geq-g_{s}\left(x, s^{-}\right) \int_{s^{-}}^{s^{+}} e^{g(x, s)} \mathrm{d} s \geq \frac{g_{s}\left(x, s^{-}\right)}{g_{s}\left(x, s^{+}\right)} e^{g\left(x, s^{-}\right)}\left[1-e^{g_{s}\left(x, s^{+}\right)\left(s^{+}-s^{-}\right)}\right] .
$$

For the part for $k=1, s^{-}=0$ and $s^{+}=|x|^{-2 / 3}$

$$
e^{g(x, 0)}\left[1-e^{g_{s}(x, 0)|x|^{-2 / 3}}\right] \geq-g_{s}(x, 0) \int_{0}^{|x|^{-2 / 3}} e^{g(x, s)} \mathrm{d} s \geq \frac{g_{s}(x, 0)}{g_{s}\left(x,|x|^{-2 / 3}\right)} e^{g(x, 0)}\left[1-e^{g_{s}\left(x,|x|^{-2 / 3}\right)|x|^{-2 / 3}}\right]
$$

Consider the limit $x \rightarrow-\infty$. Since $\lim _{x \rightarrow-\infty} g_{s}(x, 0)|x|^{-2 / 3}=\lim _{x \rightarrow-\infty} g_{s}\left(x,|x|^{-2 / 3}\right)|x|^{-2 / 3}=-\infty, \lim _{x \rightarrow-\infty} g_{s}(x, 0) / g_{s}(x,|x|$ 1 , and $\exp [g(x, 0)]=1$, this implies

$$
1 \geq \lim _{x \rightarrow-\infty}\left(-g_{s}(x, 0) \int_{0}^{|x|^{-2 / 3}} e^{g(x, s)} \mathrm{d} s\right) \geq 1
$$

Since, $\lim _{x \rightarrow-\infty} \exp \left[g\left(x,(k-1)|x|^{-2 / 3}\right)\right]=0$, a similar argument for all $k>1$ yields

$$
\lim _{x \rightarrow-\infty}\left(-g_{s}\left[x,(k-1)|x|^{-2 / 3}\right] \int_{(k-1)|x|^{-2 / 3}}^{k|x|^{-2 / 3}} e^{g(x, s)} \mathrm{d} s\right)=0 .
$$

Hence

$$
\lim _{x \rightarrow-\infty}\left(-g_{s}(x, 0) \int_{0}^{\infty} e^{g(x, s)} \mathrm{d} s\right)=\lim _{x \rightarrow-\infty}\left(-g_{s}(x, 0) a(x)\right)=1 .
$$

5. Define

$$
z:=s-\ln \frac{x}{\psi}
$$

Substitution in $g(x, s)$ and $a(x)=\int_{0}^{\infty} e^{g(x, s)} \mathrm{d} s$ yields

$$
\begin{aligned}
g\left(x, z+\ln \frac{x}{\psi}\right) & =x-\psi e^{-z}+\frac{\sigma^{2} \psi^{2}}{4}-\frac{\sigma^{2}}{4} x^{-2} e^{-2 z}-\psi\left(z+\ln \frac{x}{\psi}\right) \\
a(x) & =\exp \left(x+\frac{\sigma^{2} \psi^{2}}{4}-\psi \ln \frac{x}{\psi}\right) \int_{-\ln (x / \psi)}^{\infty} \exp \left(-\psi e^{-z}-\frac{\sigma^{2}}{4} x^{-2} e^{-2 z}-\psi z\right) \mathrm{d} z
\end{aligned}
$$


Rearranging and taking limits yields

$$
\begin{aligned}
\lim _{x \rightarrow \infty}\left[\exp \left(-x-\frac{\sigma^{2} \psi^{2}}{4}+\psi \ln \frac{x}{\psi}\right) a(x)\right] & =\lim _{x \rightarrow \infty}\left[\int_{-\ln (x / \psi)}^{\infty} \exp \left(-\psi e^{-z}-\frac{\sigma^{2}}{4} x^{-2} e^{-2 z}-\psi z\right) \mathrm{d} z\right] \\
& =\lim _{x \rightarrow \infty}\left[\psi^{\psi} \int_{0}^{x / \psi} y^{\psi-1} e^{-y} \mathrm{~d} y\right]=\psi^{\psi} \Gamma(\psi)
\end{aligned}
$$

where we apply the variable transformation $y:=\psi e^{-z}$ in the second step.

\section{A.4 A Taylor series representation of $a(x)$}

The function $a(x)$ as defined in equation (3.3) can be approximated by a Taylor expansion starting from $\left.a(x)\right|_{\sigma^{2}=0}$ as follows:

$$
\begin{aligned}
a^{(n)}(x) & :=e^{x} x^{-\psi} \gamma(\psi, x)+\left.\sum_{k=1}^{n} \frac{\sigma^{2 k}}{k !} \frac{\partial^{k} a(x)}{\left(\partial \sigma^{2}\right)^{k}}\right|_{\sigma^{2}=0}, \lim _{n \rightarrow \infty} a^{(n)}(x) \rightarrow a(x), \\
0 & <\left.\frac{\partial^{k} a(x)}{\left(\partial \sigma^{2}\right)^{k}}\right|_{\sigma^{2}=0}<\left.\frac{1}{4} \frac{\partial^{(k-1)} a(x)}{\left(\partial \sigma^{2}\right)^{(k-1)}}\right|_{\sigma^{2}=0}
\end{aligned}
$$

where the superscript $(n)$ refers to the order of approximation and where the mode of convergence is (at least pointwise) monotone because of $\left.\frac{\partial^{k} a(x)}{\left(\partial \sigma^{2}\right)^{k}}\right|_{\sigma^{2}=0}>0$. The proof uses that for two parameters $a, b \in \mathbb{R}$ and with $a>0$, we have

$$
\begin{aligned}
\left.\frac{\partial^{k} a(x)}{\left(\partial \sigma^{2}\right)^{k}}\right|_{\sigma^{2}=0}:= & 4^{-k} \int_{0}^{\infty}\left(1-e^{-2 s}\right)^{k} \exp \left[-a s+b\left(1-e^{-s}\right)\right] \mathrm{d} s \\
= & 4^{-k} \sqrt{\pi} e^{b} \Gamma[k+1]\left\{\frac{1}{2} \Gamma\left[\frac{a}{2}\right]{ }_{1} \tilde{F}_{2}\left[\frac{a}{2} ;\left(\frac{1}{2}, \frac{a}{2}+k+1\right) ; \frac{b^{2}}{4}\right]\right. \\
& \left.\quad-\frac{b}{4} \Gamma\left[\frac{a+1}{2}\right]{ }_{1} \tilde{F}_{2}\left[\frac{a+1}{2} ;\left(\frac{3}{2}, \frac{a+3}{2}+k\right) ; \frac{b^{2}}{4}\right]\right\}
\end{aligned}
$$

where ${ }_{p} \tilde{F}_{q}(a ; b ; z)$ is the regularized generalized hypergeometric function, defined as

$$
{ }_{p} \tilde{F}_{q}(a ; b ; z)={ }_{p} F_{q}(a ; b ; z) /\left(\Gamma\left[b_{1}\right] \ldots \Gamma\left[b_{q}\right]\right) .
$$

Otherwise, the proof is straightforward. This series representation is useful because the hypergeometric function can be evaluated to arbitrary precision using standard software packages. The derivatives are all positive and converge to zero quickly. Hence, this Taylor expansion converges more quickly than a Taylor expansion of the function $\exp \left(\frac{1}{4} x\right)$ with respect to $x$, since in that case the $k$-th derivative is exactly equal to the $(k-1)$-th derivative divided by four.

\section{A.5 Proof of Proposition 3}

1. Equation (3.3) and (2.1) imply

$$
\begin{aligned}
\mathrm{d} \ln A\left(x_{s}, y_{s}\right) & =\mathrm{d} \ln a\left(x_{s}\right)+\mathrm{d} \ln y_{s} \\
& =\frac{a^{\prime}}{a} \mathrm{~d} x_{s}+\frac{1}{2} \frac{a^{\prime \prime} a-a^{\prime 2}}{a^{2}} \mathrm{~d} x_{s}^{2}+\left(x_{s}+\mu+\sigma^{2}\right) \mathrm{d} s+O\left(\mathrm{~d} s^{2}\right), \\
& =\left[\mu+\left(1-\frac{a^{\prime}}{a}\right)\left(x_{s}+\sigma^{2}\right)+\frac{\sigma^{2}}{2} \frac{a^{\prime \prime} a-a^{\prime 2}}{a^{2}}\right] \mathrm{d} s+\frac{a^{\prime}}{a} \sigma \mathrm{d} W_{s}+O\left(\mathrm{~d} s^{2}\right) .
\end{aligned}
$$

The second line follows by Itô's lemma; the third line uses the expressions for $\mathrm{d} x_{s}$. For the slope of the drift with respect to $x_{s}$ : for $x_{s} \rightarrow-\infty, \lim _{x_{s} \rightarrow-\infty} a^{\prime} / a=0$ and $\lim _{x_{s} \rightarrow-\infty}\left(a^{\prime \prime} a-a^{\prime 2}\right) / a^{2}=0$, see Proposition 2 . We have not been able to provide a general proof that the drift is increasing in $x_{s}$ for all $x_{s}$ since $a$ is a function of $x_{s}$. For the slope of the variance with respect to $x_{s}: \mathrm{d}\left(a^{\prime} / a\right) / \mathrm{d} s>0$, see Proposition 2.

2. By Proposition 2, $\mathrm{d} \ln y_{s} / \mathrm{d} x_{0}$ is proportional to $1-e^{-s}$. Hence, $\mathrm{d}\left(\mathrm{E} \ln y_{0}\right) / \mathrm{d} x_{0}=0$, while $\mathrm{d}\left(\mathrm{E} \ln y_{\infty}\right) / \mathrm{d} x_{0}=1$. 
Since $\ln A(x, y)=\ln y+\ln a(x)$,

$$
\mathrm{d}\left(\mathrm{E} \ln y_{s}\right) / \mathrm{d} x_{0}=\mathrm{d}\left(\mathrm{E} \ln y_{s}\right) / \mathrm{d} x_{0}+\frac{a^{\prime}\left(\mathrm{E} x_{s}\right)}{a\left(\mathrm{E} x_{s}\right)} \frac{\mathrm{dE} x_{s}}{\mathrm{~d} x_{0}} .
$$

Since $\mathrm{dE} x_{0} / \mathrm{d} x_{0}=1$, while $\mathrm{dE} x_{s} / \mathrm{d} x_{0}=0$ (because $x_{s}$ is mean-reverting) this proves the proposition.

\section{A.6 Proof of Proposition 4}

1. Note that for $(x, y) \in \mathcal{C}$ we have $W_{\lambda}(x, y)>0$ for some large enough value of $\lambda>0$, because the sets $\mathcal{C}_{\lambda}$ are expanding to the limiting set $\mathcal{C}$ as $\lambda$ increases. Thus $W(x, y)>0$ for all $(x, y) \in \mathcal{C}$. The contrapositive statement is that $W(x, y) \leq 0$ implies $x \in \mathcal{S}$. At the same time, if $(x, y) \in \mathcal{S}$ then $W_{\lambda}(x, y) \leq 0$ for all $\lambda>0$, thus $W(x, y) \leq 0$. Together this implies $\mathcal{S}=\{(x, y): W(x) \leq 0\}$.

Next we show that the ' $\leq$ ' $\operatorname{sign}$ in $\mathcal{S}=\{(x, y): W(x) \leq 0\}$ can be replaced by a ' $=$ ' sign. The value of waiting $W_{\lambda}(x, y)$ satisfies $(\rho+\lambda-\mathcal{L}) W_{\lambda}(x, y)=(1-y)+\lambda W_{\lambda}^{+}(x, y)$, such that

$$
(\rho+\lambda-\mathcal{L}) W_{\lambda}(x, y) \geq(1-y) \quad \Rightarrow \quad W_{\lambda}(x, y) \geq(\rho+\lambda-\mathcal{L})^{-1}(1-y)
$$

The inverse $(\rho+\lambda-\mathcal{L})^{-1}$ can be defined rigorously via the resolvent formalism, see e.g. Revuz and Yor (1999) (pp. 89-90, 290-1) or Rogers and Williams (2000) (pp. 234-8). As an intuition for this formalism, consider two functions, $h_{1}(x, y)$ and $h_{2}(x, y)$, which can be interpreted as the asset value and the cash flow respectively, and two stochastic differential equations describing the evolution of $x$ and $y$ over time. The relation between both functions can be described either by a Bellman equation $\left(h_{2}(x, y)\right.$ as a function $\left.h_{1}(x, y)\right)$ or by a present value of all expected future cash flows $\left(h_{1}(x, y)\right.$ as a function of $\left.h_{2}(x, y)\right)$ :

$$
(\rho-\mathcal{L}) h_{1}(x, y)=h_{2}(x, y) \quad \Leftrightarrow \quad h_{1}\left(x_{0}, y_{0}\right)=\mathrm{E}^{\left(x_{0}, y_{0}\right)}\left[\int_{0}^{\infty} \mathrm{e}^{-\rho s} h_{2}\left(x_{s}, y_{s}\right) \mathrm{d} s\right]
$$

The equivalence of both functional equations suggests that the definition of the inverse operator $(\rho-\mathcal{L})^{-1}$ acting on the function $h_{2}(x, y)$ should be

$$
(\rho-\mathcal{L})^{-1} h_{2}\left(x_{0}, y_{0}\right):=\mathrm{E}^{\left(x_{0}, y_{0}\right)}\left[\int_{0}^{\infty} \mathrm{e}^{-\rho s} h_{2}\left(x_{s}, y_{s}\right) \mathrm{d} s\right]
$$

The inverse $(\rho-\mathcal{L})^{-1}$ is known the resolvent operator. In the sequel, we use five well-known resolvent properties discussed in e.g. Rogers and Williams (2000) (pp. 234-8):

- Linearity, that is $(\rho-\mathcal{L})^{-1}\left[h_{1}(x, y)+h_{2}(x, y)\right]=(\rho-\mathcal{L})^{-1} h_{1}(x, y)+(\rho-\mathcal{L})^{-1} h_{2}(x, y)$,

- Order-preserving property, that is $h_{1}(x, y) \geq h_{2}(x, y), \forall(x, y)$ implies $(\rho-\mathcal{L})^{-1} h_{1}(x, y) \geq(\rho-\mathcal{L})^{-1} h_{2}(x, y), \forall(x, y)$.

- Contraction, that is $0 \leq h(x, y) \leq C$ for some constant $C \geq 0$ implies $(\rho-\mathcal{L})^{-1} h(x, y) \leq C / \rho$,

- Semi-group property, that is $\lim _{\lambda \rightarrow \infty} \lambda(\rho+\lambda-\mathcal{L})^{-1} h(x, y)=h(x, y)$ if $h(x, y)$ is a continuous function,

- Hille-Yosida property, that is $\lim _{\lambda \rightarrow \infty} \lambda\left[1-\lambda(\rho+\lambda-\mathcal{L})^{-1}\right] h(x, y)=(\rho-\mathcal{L}) h(x, y)$ as long as $\mathcal{L} h(x, y)$ exists.

The first four follow directly from the definition of the resolvent in (A.6). The Hille-Yosida property can be understood intuitively by using the Taylor expansion $\lambda(\rho+\lambda-\mathcal{L})^{-1}=1-(\rho-\mathcal{L}) / \lambda+O\left(\lambda^{-2}\right)$, valid for large $\lambda$, inside the square brackets.

Let us now return to equation (A.5). For $(\rho+\lambda-\mathcal{L})^{-1}$ with $\rho+\lambda>0$ operating on the function $1-y$ to exist, it is sufficient that $\mathrm{e}^{-\rho s} \mathrm{E}\left(y_{s}\right)$ is bounded for all $s \geq 0$. Recall that, asymptotically, $y_{s}$ grows at the (exponential) rate $\mu$; see Proposition 1. This is strictly slower than $\rho+\lambda$ for all $\lambda>0$, because $\mu<\rho$; see equation (3.4). Hence $(\rho+\lambda-\mathcal{L})^{-1}(1-y)$ exists for all $\lambda>0$. Since $W_{\lambda}(x, y)$ is non-decreasing in $\lambda$, it follows from (A.5) that

$$
(\rho+\lambda-\mathcal{L})^{-1}(1-y) \leq W_{\lambda}(x, y) \leq W(x, y) \leq 0, \quad(x, y) \in \mathcal{S} \subseteq \mathcal{S}_{\lambda}
$$

where the last inequality holds because $\mathcal{S}=\{(x, y): W(x) \leq 0\}$, as derived above. In the limit $\lambda \rightarrow \infty$, the left-hand side of this equation converges to zero. The sandwich (A.7) thus gives $W(x, y)=0$ on $\mathcal{S}$. This completes the proof of part one of Proposition 4. 
2. Apart from the last inequality, equation (A.7) holds for all $(x, y) \in \mathbb{R} \times \mathbb{R}_{\geq 0}$, that is

$$
(\rho+\lambda-\mathcal{L})^{-1}(1-y) \leq W_{\lambda}(x, y) \leq W(x, y), \quad \forall(x, y) \in \mathbb{R} \times \mathbb{R}_{\geq 0} .
$$

If $(\rho+\lambda-\mathcal{L})^{-1}(1-y)>0$ for some point $(x, y)$, then $W(x, y)>0$, and thus $(x, y) \in \mathcal{C}$, since $\mathcal{C}:=\{(x, y) \in$ $\left.\mathbb{R} \times \mathbb{R}_{\geq 0}: W(x, y)>0\right\}$. In other words, $(\rho+\lambda-\mathcal{L})^{-1}(1-y)>0$ is a sufficient condition for continuing. To see what this means, we multiply by $\lambda$ and consider $\lambda(\rho+\lambda-\mathcal{L})^{-1}(1-y)>0$ in the limit where $\lambda \rightarrow \infty$. We have already established (in part one above) that $(\rho+\lambda-\mathcal{L})^{-1}(1-y)$ exists for all $\lambda>0$. Furthermore, the semi-group property of the resolvent formalism (see references above) tells us that $\lambda(\rho+\lambda-\mathcal{L})^{-1}$ converges to the identity function in the limit as $\lambda \rightarrow \infty$. (For this result it is important that $1-y$ is a continuous function.) We thus have the implication $1-y>0 \Rightarrow(x, y) \in \mathcal{C}$, which is the first statement in part two of Proposition 4. Conversely, the sufficient condition for continuing cannot be satisfied on $\mathcal{S}$, hence $(x, y) \in \mathcal{S}$ implies $1-y \leq 0$.

3. To demonstrate the pointwise convergence on $\mathcal{C}$, fix some location $(x, y) \in \mathcal{C}$. Recall that $W(x, y) \geq W_{\lambda}(x, y)>0$ for all $(x, y) \in \mathcal{C}_{\lambda}$. This means that there is exists some $\lambda_{\min }$, such that $W_{\lambda}(x, y)>0$ for all $\lambda>\lambda_{\min }$. Since $W_{\lambda}(x, y)$ is strictly positive on $\mathcal{C}$ for sufficiently large $\lambda$, it holds that the negative part of $W_{\lambda}(x, y)$, that is $W_{\lambda}^{-}(x, y)$, is identically zero on $\mathcal{C}$ for these sufficient large values of $\lambda$. This gives the first part of (4.9).

To prove the second part of (4.9), we first note that $\lambda W_{\lambda}^{-}(x, y)$ can be written as

$$
\begin{aligned}
\lambda W_{\lambda}^{-}(x, y) & =\max \left\{0,-\lambda W_{\lambda}(x, y)\right\} \\
& =\max \left\{0, \lambda(\rho+\lambda-\mathcal{L})^{-1}(y-1)-\lambda^{2}(\rho+\lambda-\mathcal{L})^{-1} W_{\lambda}^{+}(x, y)\right\} \\
& \rightarrow \max \{0, y-1\}, \quad \forall(x, y) \in \mathcal{S} \backslash \partial \mathcal{S}, \\
& =y-1, \quad \forall(x, y) \in \mathcal{S} \backslash \partial \mathcal{S}
\end{aligned}
$$

We separately consider each step of the above calculation. The second line holds because

$$
(\rho+\lambda-\mathcal{L}) W_{\lambda}(x, y)=(1-y)+\lambda W_{\lambda}^{+}(x, y)
$$

The limit in the third line holds because

$$
\lambda(\rho+\lambda-\mathcal{L})^{-1}(y-1) \rightarrow y-1 \quad \text { as } \quad \lambda \rightarrow \infty
$$

which follows by again by the semi-group property of the resolvent. For the other term inside the max operator, we have

$$
\begin{aligned}
0 \leq \lambda^{2}(\rho+\lambda-\mathcal{L})^{-1} W_{\lambda}^{+}(x, y) & \leq \lambda^{2}(\rho+\lambda-\mathcal{L})^{-1} W(x, y) & & \\
& =\lambda\left[\lambda(\rho+\lambda-\mathcal{L})^{-1}-1\right] W(x, y), & & (x, y) \in \mathcal{S} \backslash \partial \mathcal{S}, \\
& \rightarrow(\mathcal{L}-\rho) W(x, y), & & (x, y) \in \mathcal{S} \backslash \partial \mathcal{S}, \\
& =0, & & (x, y) \in \mathcal{S} \backslash \partial \mathcal{S} .
\end{aligned}
$$

The first line holds because $0 \leq W_{\lambda}^{+}(x, y) \leq W^{+}(x, y)=W(x, y)$ by the order-preserving property of the resolvent. The second line holds because $W(x, y)$ is identically zero on $\mathcal{S} \backslash \partial \mathcal{S}$. The limit in the third line follows by the Hille-Yosida property of the resolvent. The last equality holds because $W(x, y)$ is identically zero on the interior of $\mathcal{S}$, such that $(\mathcal{L}-\rho) W(x, y)=0$. Comparing the first and last lines of the above calculation, it follows that $\lim _{\lambda \rightarrow \infty} \lambda^{2}(\rho+\lambda-\mathcal{L})^{-1} W_{\lambda}^{+}(x, y)$ is bounded above and below by zero. Hence the third line in (A.8) follows. ${ }^{26}$ Finally, the last line in (A.8) follows because $(x, y) \in \mathcal{S} \backslash \partial \mathcal{S}$ implies $y-1 \geq 0$ (recall part one of the proof). This completes the proof of Proposition 4.

\section{A.7 Proof of Proposition 5}

The value of waiting satisfies $(\rho-\mathcal{L}) W_{\lambda}(x, y)=1-y+\lambda W_{\lambda}^{-}(x, y)$ or, what is equivalent,

$$
W_{\lambda}(x, y)=(\rho-\mathcal{L})^{-1}\left[1-y+\lambda W_{\lambda}^{-}\right]
$$

\footnotetext{
${ }^{26}$ The present argument be generalised to all stochastic processes with continuous sample paths; see e.g. Lange et al. (in prep) who provide a generalisation of the classic Hille-Yosida property of the resolvent. The key point is to take $(x, y)$ in the interior of $\mathcal{S}$, i.e. away from the boundary $\partial \mathcal{S}$, such that the function of interest, in this case $W(x, y)$, is identically zero in a little ball around the point $(x, y)$.
} 
Taking the limit $\lambda \rightarrow \infty$, we have $W_{\lambda}(x, y) \rightarrow W(x, y)$ in a pointwise sense on the left. The crucial part of the proof is to show that we may may push the limit $\lambda \rightarrow \infty$ through the resolvent operator $(\rho-\mathcal{L})^{-1}$ on the right-hand side. From the definition of the resolvent in (A.6), we know that $(\rho-\mathcal{L})^{-1}$ must be viewed as an integral operator with respect to the probability density of the stochastic process, as well as with respect to time. We must therefore show that Lebesgue's dominated convergence theorem applies. When this is established, the result in Proposition 5 follows almost directly. To see why, recall that Proposition 4 showed

$$
\lambda W_{\lambda}^{-}(x, y) \rightarrow \begin{cases}0, & (x, y) \in \mathcal{C} \\ y-1 \geq 0, & (x, y) \in \mathcal{S} \backslash \partial \mathcal{S}\end{cases}
$$

Under the relatively mild assumption that $\partial \mathcal{S}$ is of Lebesgue measure zero, taking the limit $\lambda \rightarrow \infty$ under the resolvent $(\rho-\mathcal{L})^{-1}$ in equation $($ A.9) gives

$$
W(x, y):=\lim _{\lambda \rightarrow \infty} W_{\lambda}(x, y)=\lim _{\lambda \rightarrow \infty}(\rho-\mathcal{L})^{-1}\left[1-y+\lambda W_{\lambda}^{-}\right]=(\rho-\mathcal{L})^{-1}\left[\mathbb{1}_{\mathcal{C}}(1-y)\right]
$$

where $\mathbb{1}_{\mathcal{C}}$ is the indicator function of the set $\mathcal{C}$, which equals one if $(x, y) \in \mathcal{C}$ and zero otherwise. Finally, we use the representation of the resolvent as in (A.6) to obtain

$$
W(x, y)=(\rho-\mathcal{L})^{-1}\left[\mathbb{1}_{\mathcal{C}}(1-y)\right]=\int_{0}^{\infty} \mathrm{e}^{-\rho s} \mathrm{E}_{(x, y)}\left[\mathbb{1}_{\mathcal{C}}(1-y)\right] \mathrm{d} s
$$

which is the desired result. It only remains to be shown that Lebesgue's dominated convergence theorem applies. For this purpose, assume the point $(x, y)$ to be fixed.

First, we consider $(\rho-\mathcal{L})^{-1}[1-y]$ on the right-hand side of equation (A.9). This term is bounded because

$$
(\rho-\mathcal{L})^{-1}(1-y)=\rho^{-1}-(\rho-\mathcal{L})^{-1} y
$$

The quantity $(\rho-\mathcal{L})^{-1} y$ is finite because $y_{s}$ grows asymptotically at the (exponential) rate $\mu$, which is assumed to be strictly smaller than the discount rate $\rho$, i.e. $\mu<\rho$. We use this fact several times below.

Second, we consider $(\rho-\mathcal{L})^{-1}\left[\lambda W_{\lambda}^{-}\right]$on the right-hand side of equation (A.9). The penalty term $\lambda W_{\lambda}^{-}(x, y)$, which appears under the resolvent $(\rho-\mathcal{L})^{-1}$, remains bounded because

$$
\begin{aligned}
\lambda W_{\lambda}^{-}(x, y) & =\max \left\{0,-\lambda W_{\lambda}(x, y)\right\} \\
& =\max \left\{0, \lambda(\rho+\lambda-\mathcal{L})^{-1}(y-1)-\lambda^{2}(\rho+\lambda-\mathcal{L})^{-1} W_{\lambda}^{+}(x, y)\right\} \\
& \left.\leq \max \left\{0, \lambda(\rho+\lambda-\mathcal{L})^{-1}(y-1)\right)\right\} \\
& \rightarrow \max \{0, y-1\}
\end{aligned}
$$

The second line holds because $(\rho+\lambda-\mathcal{L}) W_{\lambda}(x, y)=1-y+\lambda W_{\lambda}^{+}(x, y)$. The third line holds because we disregard the term $0 \leq \lambda^{2}(\rho+\lambda-\mathcal{L})^{-1} W_{\lambda}^{+}(x, y)$. The convergence in line four holds because $y-1$ is a continuous function. The resolvent $(\rho-\mathcal{L})^{-1}$ can act on this function because $y_{s}$ grows asymptotically at the (exponential) rate $\mu<\rho$. We conclude that the penalty term $\lambda W_{\lambda}^{-}(x, y)$ remains bounded for all bounded $y$.

Now we consider the entire term $(\rho-\mathcal{L})^{-1}\left[\lambda W_{\lambda}^{-}\right]$on the right-hand side of equation (A.9), which remains bounded as $\lambda \rightarrow \infty$ because

$$
\begin{aligned}
0 \leq \lim _{\lambda \rightarrow \infty}(\rho-\mathcal{L})^{-1}\left[\lambda W_{\lambda}^{-}(x, y)\right] & \left.\leq \lim _{\lambda \rightarrow \infty}(\rho-\mathcal{L})^{-1} \max \left\{0, \lambda(\rho+\lambda-\mathcal{L})^{-1}(y-1)\right)\right\} \\
& \left.\leq \lim _{\lambda \rightarrow \infty}(\rho-\mathcal{L})^{-1} \max \left\{0, \lambda(\rho+\lambda-\mathcal{L})^{-1} \max \{0, y-1\}\right)\right\} \\
& =\lim _{\lambda \rightarrow \infty}(\rho-\mathcal{L})^{-1} \lambda(\rho+\lambda-\mathcal{L})^{-1} \max \{0, y-1\} \\
& =(\rho-\mathcal{L})^{-1} \max \{0, y-1\}<\infty
\end{aligned}
$$

The first line holds because the penalty term is non-negative and because $\lambda W_{\lambda}^{-}(x, y)$ can be bounded by the above arguments. The second line holds because $y-1 \leq \max \{0, y-1\}$. The third line holds because $0 \leq \lambda(\rho+\lambda-\mathcal{L})^{-1} \max \{0, y-1\}$. Finally, the fourth line holds because $\lambda(\rho+\lambda-\mathcal{L})^{-1}$ goes to the identity operator by the semi-group property. The result is again bounded for fixed locations $(x, y)$, because $y_{s}$ grows asymptotically at the (exponential) rate $\mu<\rho$.

Having shown that the right-hand side of (A.9) remains bounded for $(x, y)$ in bounded sets, we may consider its pointwise limit. We may look under the resolvent to see what happens as $\lambda \rightarrow \infty$ for an arbitrary but fixed location 
$(x, y) \in \mathbb{R} \times \mathbb{R}_{\geq 0}$. Since the boundary of $\mathcal{S}$ is assumed to have zero Lebesgue measure, the convergence of the penalty term in Proposition 4 gives us the pointwise limit of $\lambda W_{\lambda}^{-}(x, y)$ for almost all points $(x, y)$. Lebesgue's dominated convergence theorem then gives the desired result.

The resulting function $W(x, y)$ given in Proposition 4 is continuous under our standing assumption that the boundary $\partial \mathcal{S}$ has measure zero. Under this assumption, it follows that the indicator function in the integrand can be approximated by a family of lower- or upper semicontinuous functions. The resolvent preserves both forms of semi-continuity, such that $W(x, y)$ is both lower- and upper-semicontinuous, hence continuous.

Since $\mathcal{L}$ is a differential operator of second and first order in $x$ and $y$, respectively, it follows that the resolvent operator $(\rho-\mathcal{L})^{-1}$ does the exact opposite: it adds two and one orders of differentiability in the $x$ and $y$ directions, respectively. (The details are omitted, but this can be verified explicitly by using the integral representation of the resolvent together with the law of the process $\left(x_{s}, y_{s}\right)$ as derived in Proposition 1.) In this case, the function $(1-y) \mathbb{1}_{\mathcal{C}}(x, y)$ under the resolvent is discontinuous across the boundary $\partial \mathcal{S}$. Adding two orders of differentiability in the $x$ direction implies $W(x, y)$ is once continuously differentiable in $x$. For the $y$ direction, however, the same does not hold; here, we only add one order of differentiability. Hence the resulting function $W(x, y)$ is merely continuous in the $y$ direction.

Finally, since $W_{\lambda}(x, y)$ is a monotonic non-decreasing and since the pointwise limit $W(x, y)$ is continuous, Dini's theorem ensures that convergence is uniform on bounded sets.

\section{A.8 Proof of Proposition 6}

First we note that $W_{\lambda}^{(1)}(x, y)$ is bounded above, because

$$
W_{\lambda}^{(1)}(x, y):=(\rho+\lambda-\mathcal{L})^{-1}(1-y) \leq(\rho+\lambda-\mathcal{L})^{-1} 1=(\rho+\lambda)^{-1}<\infty
$$

The bound follows because from the contraction property of the resolvent, because function under the resolvent $(\rho+\lambda-\mathcal{L})^{-1}$, that is $(1-y)$, is bounded above by the constant function one. To show non-decreasing monotonicity of the sequence $\left\{W_{\lambda}^{(n)}\right\}_{n \in \mathbb{N}}$, we use the fact that the resolvent operator $(\rho+\lambda-\mathcal{L})^{-1}$ is linear and order-preserving. Thus

$$
W_{\lambda}^{(2)}(x, y):=(\rho+\lambda-\mathcal{L})^{-1}\left(1-y+\lambda\left[W_{\lambda}^{(1)}(x, y)\right]^{+}\right) \geq(\rho+\lambda-\mathcal{L})^{-1}(1-y)=: W_{\lambda}^{(1)}(x, y) .
$$

Thus the algorithm is non-decreasing at the first step. It is useful to define $W_{\lambda}^{(0)}(x, y):=0$, so that the above statement can be equivalently written for $n=1$ as follows:

$$
\begin{aligned}
W_{\lambda}^{(n+1)}(x, y) & :=(\rho+\lambda-\mathcal{L})^{-1}\left(1-y+\lambda\left[W_{\lambda}^{(n)}(x, y)\right]^{+}\right) \\
& \geq(\rho+\lambda-\mathcal{L})^{-1}\left(1-y+\lambda\left[W_{\lambda}^{(n-1)}(x, y)\right]^{+}\right)=: \quad W_{\lambda}^{(n)}(x, y)
\end{aligned}
$$

Because this statement holds for $n=1$, it also holds for $n=2$ and hence, by induction, it holds for all $n \in \mathbb{N}$, such that non-decreasing monotonicity of $\left\{W_{\lambda}^{(n)}\right\}_{n \in \mathbb{N}}$ is established.

Having shown the algorithm is non-decreasing, we show that it is convergent by using the contractive property. To prove that the iterations in Proposition 6 define a contraction, we define

$$
\begin{aligned}
0 \leq \Delta_{\lambda}^{(n+1)}(x, y) & :=W_{\lambda}^{(n+1)}(x, y)-W_{\lambda}^{(n)}(x, y) \\
& =\lambda(\rho+\lambda-\mathcal{L})^{-1}\left(\left[W_{\lambda}^{(n)}(x, y)\right]^{+}-\left[W_{\lambda}^{(n-1)}(x, y)\right]^{+}\right)
\end{aligned}
$$

with $\Delta_{\lambda}^{(1)}(x, y):=W_{\lambda}^{(1)}(x, y)$ since $W_{\lambda}^{(0)}(x, y)=0$. Next, note that

$$
0 \leq\left[W_{\lambda}^{(n)}(x, y)\right]^{+}-\left[W_{\lambda}^{(n-1)}(x, y)\right]^{+} \leq W_{\lambda}^{(n)}(x, y)-W_{\lambda}^{(n-1)}(x, y)=\Delta_{\lambda}^{(n)}(x, y)
$$

It follows that

$$
0 \leq \Delta_{\lambda}^{(n+1)}(x, y)=\lambda(\rho+\lambda-\mathcal{L})^{-1}\left(\left[W_{\lambda}^{(n)}(x, y)\right]^{+}-\left[W_{\lambda}^{(n-1)}(x, y)\right]^{+}\right) \leq \lambda(\rho+\lambda-\mathcal{L})^{-1} \Delta_{\lambda}^{(n)}(x, y)
$$

By the contraction property of the resolvent, $\Delta_{\lambda}^{(n+1)}(x, y)$ cannot exceed the global maximum of the function $\lambda /(\rho+$ $\lambda) \Delta_{\lambda}^{(n)}(x, y)$, which, in turn, cannot exceed the global maximum of $\lambda^{2} /(\rho+\lambda)^{2} \Delta_{\lambda}^{(n-1)}(x, y)$, etcetera. Since $\Delta_{\lambda}^{(1)}(x, y)$ is 
bounded above by $(\rho+\lambda)^{-1}$, this implies

$$
\Delta_{\lambda}^{(n+1)}(x, y) \leq\left(\frac{\lambda}{\rho+\lambda}\right)^{n}(\rho+\lambda)^{-1}
$$

Hence

$$
\begin{aligned}
W_{\lambda}(x, y)=W_{\lambda}^{(1)}(x, y)+\sum_{k=1}^{\infty} \Delta_{\lambda}^{(k+1)}(x, y) & \leq(\rho+\lambda)^{-1}+\sum_{k=1}^{\infty}\left(\frac{\lambda}{\rho+\lambda}\right)^{k}(\rho+\lambda)^{-1} \\
& =\sum_{k=0}^{\infty}\left(\frac{\lambda}{\rho+\lambda}\right)^{k}(\rho+\lambda)^{-1} \\
& =\frac{1}{1-\frac{\lambda}{\rho+\lambda}} \frac{1}{\rho+\lambda}=\rho^{-1} .
\end{aligned}
$$

Hence the sequence $\left\{W_{\lambda}^{(n)}\right\}_{n \in \mathbb{N}}$ is monotone non-decreasing and bounded above by the constant function $\rho^{-1}$.

\section{A.9 Proof of Proposition 7}

Recall that the infinity norm of a matrix $\mathbf{A}$, which is defined as the maximum of the absolute row sums, i.e. $\|\mathbf{A}\|_{\infty}=$ $\max _{i} \sum_{j}\left|A_{i j}\right|$. Recall also that a matrix is said to be diagonally dominant if for every row of the matrix, the absolute value of the diagonal element is weakly greater than the sum of the absolute values of the off-diagonal elements. Now let $\mathbf{L}$ be a weakly diagonally dominant matrix with non-positive diagonal elements and non-negative off-diagonal elements. Then:

1. for any $\rho, \lambda>0$, the matrix $(\rho+\lambda) \mathbf{I}-\mathbf{L}$ is invertible, $[(\rho+\lambda) \mathbf{I}-\mathbf{L}]^{-1}$ contains non-negative entries and the infinity norm of $[(\rho+\lambda) \mathbf{I}-\mathbf{L}]^{-1}$ does not exceed $(\rho+\lambda)^{-1}$.

2. for any $\rho, \lambda>0$, the iterative scheme (4.16) generates a sequence that is monotonically non-decreasing for $n \geq 1$, i.e., $\underline{W}_{\lambda}^{(n)} \geq \underline{W}_{\lambda}^{(n-1)}$ and convergent to a solution $\underline{W}_{\lambda}$ of the problem (4.15).

Both statements are implied by classic matrix properties. Part 1 uses that $(\rho+\lambda) \mathbf{I}-\mathbf{L}$ is a $Z$-matrix (defined as matrix with all off-diagonal elements non-positive) as well as an $M$-matrix (defined as a $Z$-matrix whose eigenvalues have positive real parts). From Plemmons (1977), it follows that all elements of the inverse of an $M$-matrix are non-negative. The bound on the norm of the inverse follows from Ahlberg and Nilson (1963). Part 2 relies entirely on the fact that $[(\rho+\lambda) \mathbf{I}-\mathbf{L}]^{-1}$ is an order-preserving operator with a norm bounded above by $(\rho+\lambda)^{-1}$; the order-preserving property of $[(\rho+\lambda) \mathbf{I}-\mathbf{L}]^{-1}$ gives us the monotonicity of the sequence in (4.16), while the norm gives us the contraction.

\section{A.10 Disretization of $\mathrm{L}$}

In Proposition 7, the question that remains is how to choose the $N^{2} \times N^{2}$ matrix $\mathbf{L}$ such it is weakly diagonally dominant with non-positive diagonal elements and non-negative off-diagonal elements. For the infinitesimal generator $\mathcal{L}$ defined in equation (4.4), the five-point stencil below generates a discretization $\mathbf{L}$ that satisfies these requirements:

\begin{tabular}{c|c|c} 
& $\frac{y\left(x+\mu+\sigma^{2}\right)^{+}}{\Delta_{y}}$ & \\
\hline$\frac{\sigma^{2}}{2 \Delta_{x}^{2}}+\frac{\left(x+\sigma^{2}\right)^{-}}{\Delta_{x}}$ & $-\frac{\sigma^{2}}{\Delta_{x}^{2}}-\frac{\left(x+\sigma^{2}\right)^{+}}{\Delta_{x}}-\frac{\left(x+\sigma^{2}\right)^{-}}{\Delta_{x}}$ & $\frac{\sigma^{2}}{2 \Delta_{x}^{2}}+\frac{\left(x+\sigma^{2}\right)^{+}}{\Delta_{x}}$ \\
\hline & $-\frac{y\left(x+\mu+\sigma^{2}\right)^{-}}{\Delta_{y}}-\frac{y\left(x+\mu+\sigma^{2}\right)^{+}}{\Delta_{y}}$ & \\
\hline & $\frac{y\left(x+\mu+\sigma^{2}\right)^{-}}{\Delta_{y}}$ &
\end{tabular}

where $(\cdot)^{+}=\max \{0, \cdot\},(\cdot)^{-}=\max \{0,-\cdot\}$, while $\Delta_{x}$ and $\Delta_{y}$ denote the horizontal and vertical spacing of $x$ and $y$. This stencil satisfies the assumptions above, since the center value, which is placed on the diagonal of $\mathbf{L}$, is constructed to be non-positive, while the values corresponding to its four neighbors, which are placed on the off diagonal elements of $\mathbf{L}$, are constructed to be non-negative. Diagonal dominance of $\mathbf{L}$ follows trivially, since the value in the center, which ends up on the diagonal of $\mathbf{L}$, is not exceeded in absolute value by sum of the other values in the stencil.

In the approximation of second derivatives with respect to $x$, we have used a central difference scheme, which uses grid points to the left and right of the center point. In the approximation of first derivatives with respect to $x$, in contrast, we use either a 'forward' or 'backward' approximation. This means that, in addition to the center point, we use either the leftor right-hand neighbor, but never both. Which one is chosen depends on the direction of the drift, such that the nearest neighbor on the right gets a positive value if the drift is towards the right. The same reasoning is applied to derivative with respect to $y$, and this leads to the desirable result that negative values are guaranteed to end up at the center of the stencil. 
While forward and backward approximations of derivatives are only first-order accurate in the grid spacing, the resulting 'upwind' scheme guarantees numerical stability, which is our main concern here.

We must also consider boundary conditions. When we reach the edge of our grid, some points in our stencil may not be 'available'. One method for dealing with such 'ghost points' besides the grid is simply to ignore the stencil value corresponding to the non-existent neighbor, which leads to Dirichlet boundary conditions. Alternatively, the stencil value corresponding to the non-existent neighbor may be re-assigned to the center value, leading to Neumann boundary conditions. In our numerical analysis, using Dirichlet or Neumann boundary conditions makes no noticeable difference.

Finally, a note on numerical efficiency. The vector $\underline{W}_{\lambda}^{(n)}$ in equation (4.16) may be obtained from the vector $\underline{W}_{\lambda}^{(n-1)}$ by explicitly computing the inverse matrix $[(\rho+\lambda) \mathbf{I}-\mathbf{L}]^{-1}$. However, this is computationally inefficient, because the $N^{2} \times N^{2}$ matrix $(\rho+\lambda) \mathbf{I}-\mathbf{L}$ is sparse - containing fewer than $5 N^{2}$ non-zero entries when generated by the five-point stencil above - while its inverse is dense. Hence, it is computationally more efficient to use 'implicit' sparse linear algebra techniques to solve the $N^{2}$ equations in (4.16).

\section{A.11 Derivation of $(5.8)$}

Using $B^{*}(x, y)=\widetilde{\alpha}[\phi A(x, y)]^{1 /(1-\alpha)}$, we write

$$
(\mathcal{L}-\rho)(1-\alpha) B^{*}(x, y)=(1-\alpha) \widetilde{\alpha}(\mathcal{L}-\rho)[\phi A(x, y)]^{1 /(1-\alpha)} .
$$

We compute $\mathcal{L} A^{1 /(1-\alpha)}(x, y)$ using the chain rule

$$
\begin{aligned}
\mathcal{L} A(x, y)^{1 /(1-\alpha)}= & \frac{1}{1-\alpha} A(x, y)^{\alpha /(1-\alpha)} \mathcal{L} A(x, y) \\
& +\frac{\sigma^{2}}{2} \frac{\alpha}{(1-\alpha)^{2}} A(x, y)^{1 /(1-\alpha)}\left(\frac{A_{x}(x, y)}{A(x, y)}\right)^{2}
\end{aligned}
$$

where the second term on the right-hand side appears because $\mathcal{L}$ contains a second-order derivative with respect to $x$. Substitution of (A.12) into (A.11) and using $\mathcal{L} A(x, y)=\rho A(x, y)-y$ as in (3.2) and $A(x, y)=y a(x)$ as in (3.3) gives

$$
\begin{aligned}
& (1-\alpha) \widetilde{\alpha}(\mathcal{L}-\rho)[\phi A(x, y)]^{1 /(1-\alpha)} \\
& =\quad \widetilde{\alpha} \phi[\phi y a(x)]^{\alpha /(1-\alpha)}[\rho y a(x)-y]+\frac{\sigma^{2}}{2} \frac{\alpha^{1 /(1-\alpha)}}{1-\alpha}[\phi y a(x)]^{1 /(1-\alpha)}\left(\frac{a_{x}(x)}{a(x)}\right)^{2} \\
& \quad-\rho \widetilde{\alpha}(\not 1-\alpha)[\phi y a(x)]^{1 /(1-\alpha)} .
\end{aligned}
$$

Two terms (as indicated by the strike-outs) cancel and the factor

$$
\widetilde{\alpha}[\phi y]^{1 /(1-\alpha)} a(x)^{\alpha /(1-\alpha)}=\phi y[\alpha \phi y a(x)]^{\alpha /(1-\alpha)}=\phi y \mathcal{K}^{*}(x, y)^{\alpha}
$$

can be pulled outside brackets, to obtain

$$
\begin{aligned}
& (1-\alpha) \widetilde{\alpha}(\mathcal{L}-\rho)[\phi A(x, y)]^{1 /(1-\alpha)}= \\
& \quad-\phi y[\alpha \phi y a(x)]^{\alpha /(1-\alpha)}\left[1-\rho \alpha a(x)-\frac{\sigma^{2}}{2} \frac{\alpha}{1-\alpha} \frac{a_{x}(x)^{2}}{a(x)}\right],
\end{aligned}
$$

which is equivalent to $(5.8)$.

\section{A.12 Proof of Proposition 8}

1. Optimal investment does not depend on $y$ : We conjecture that the option value of the project $V(x, y)$ can be written

$$
V(x, y)=y^{1 /(1-\alpha)} \mathrm{e}^{x /(1-\alpha)} v(x), \quad \forall(x, y) \in \mathcal{C} .
$$

For this conjecture to be valid, it must solve the Bellman equation $(\rho-\mathcal{L}) V(x, y)=0$, see equation (5.7). Several lines of algebra gives the following ODE for $v(x)$ :

$$
0=\frac{\sigma^{2}}{2} v^{\prime \prime}(x)-\left(x-\frac{\alpha \sigma^{2}}{1-\alpha}\right) v^{\prime}(x)+\nu v(x), \quad \forall x \in \mathbb{R},
$$


where $\nu:=\frac{\mu}{1-\alpha}+\frac{\sigma^{2}}{2(1-\alpha)^{2}}-\rho<0$ is strictly negative; recall our assumption (5.6). This is an ordinary differential equation (ODE) of $v(x)$; it no longer depends on $y$. Thus our conjecture is a possible solution to the Bellman equation as long as $v(x)$ satisfies this ODE. Appendix A.13 shows that this ODE has two independent solutions. However, the boundary condition at $x \rightarrow \infty$ requires that we take a particular linear combination of both solutions. This leaves one overall multiplicative constant, which we denote by $C$. Hence there are two remaining unknowns: the multiplicative constant $C$ and the boundary point $x^{*}$. These two constants can be determined jointly by requiring that the solution must satisfy value matching and smooth pasting conditions, which, by equation (5.5), read as follows:

$$
\begin{array}{rlrl}
V(x, y) & =\phi \mathcal{K}^{*}(x, y)^{\alpha} A(x, y)-\mathcal{K}^{*}(x, y), & \\
& =(1-\alpha) \widetilde{\alpha}[\phi A(x, y)]^{1 /(1-\alpha)}, & & \\
V_{x}(x, y) & =\widetilde{\alpha}[\phi A(x, y) \in \partial \mathcal{S}, \\
& =\frac{V(x, y)}{1-\alpha} \frac{A_{x}(x, y)}{A(x, y)}, & & \forall(x, y) \in \partial \mathcal{S},
\end{array}
$$

If the boundary $\partial \mathcal{S}$ consists of a single point denoted by $x^{*}$, then we may rewrite these conditions using $A(x, y)=$ $a(x) y$ and equation (A.13) to yield

$$
\begin{aligned}
\mathrm{e}^{x^{*} /(1-\alpha)} v\left(x^{*}\right) & =(1-\alpha) \widetilde{\alpha}\left[\phi a\left(x^{*}\right)\right]^{1 /(1-\alpha)}, \\
\mathrm{e}^{x^{*} /(1-\alpha)}\left[(1-\alpha) v\left(x^{*}\right)+v^{\prime}\left(x^{*}\right)\right] & =\widetilde{\alpha} \phi^{1 /(1-\alpha)} a\left(x^{*}\right)^{\alpha /(1-\alpha)} a^{\prime}\left(x^{*}\right), \\
& =\frac{\mathrm{e}^{x^{*} /(1-\alpha)} v\left(x^{*}\right)}{1-\alpha} \frac{a^{\prime}\left(x^{*}\right)}{a\left(x^{*}\right)}
\end{aligned}
$$

The smooth pasting condition can be further simplified to read

$$
(1-\alpha)^{2}=\frac{a^{\prime}\left(x^{*}\right)}{a\left(x^{*}\right)}-(1-\alpha) \frac{v^{\prime}\left(x^{*}\right)}{v\left(x^{*}\right)}
$$

2. Critical upper bound for $x$ : Consider again the value of the project when investment is fixed at $\mathcal{K}$ and when investment can be set at its optimal value $\mathcal{K}^{*}(x, y)$ :

$$
\begin{aligned}
\phi \mathcal{K}^{\alpha} A(x, y)-\mathcal{K}, & \forall(x, y) \in \mathbb{R} \times \mathbb{R}_{\geq 0}, \\
(1-\alpha) \widetilde{\alpha}[\phi A(x, y)]^{1 /(1-\alpha)}, & \forall(x, y) \in \mathbb{R} \times \mathbb{R}_{\geq 0} .
\end{aligned}
$$

The value matching condition stipulates that both should be equal at $(x, y) \in \partial \mathcal{S}$. Moreover, the investor should wait (i.e. not invest) if the expected return on the (optimally scaled) asset $(1-\alpha) \tilde{\alpha}[\phi A(x, y)]^{\alpha /(1-\alpha)}$ is larger than the risk-free return $\rho$ on this asset. Thus the following implication holds:

$$
(1-\alpha) \widetilde{\alpha}(\mathcal{L}-\rho)[\phi A(x, y)]^{1 /(1-\alpha)}>0 \quad \Rightarrow(x, y) \in \mathcal{C} .
$$

(The converse statement is not true.) Given that the left-hand side is continuous, we may also investigate the implication

$$
0=(\mathcal{L}-\rho) A(x, y)^{1 /(1-\alpha)} \quad \Rightarrow(x, y) \in \mathcal{C} .
$$

We will find this equation to define a curve in $(x, y)$ space given by $x=$ constant. Substitution of (A.12) for $\mathcal{L} A(x, y)^{1 /(1-\alpha)}$ and using $\mathcal{L} A(x, y)=\rho A(x, y)-y$ as in (3.2) and $A(x, y)=y a(x)$ as in (3.3) gives

$$
\begin{aligned}
0= & \frac{1}{1-\alpha}[y a(x)]^{\alpha /(1-\alpha)}[\rho y a(x)-y]-\rho[y a(x)]^{1 /(1-\alpha)} \\
& +\frac{\sigma^{2}}{2} \frac{\alpha}{(1-\alpha)^{2}}[y a(x)]^{1 /(1-\alpha)}\left(\frac{a_{x}(x)}{a(x)}\right)^{2} .
\end{aligned}
$$

This equation can be simplified ${ }^{27}$ to give

$$
1=\rho \alpha a(x)+\frac{\sigma^{2}}{2} \frac{\alpha}{1-\alpha} \frac{a_{x}(x)^{2}}{a(x)}
$$

\footnotetext{
${ }^{27}$ We multiply by $1-\alpha$, after which two terms cancel, we recognise that the factor $y^{1 /(1-\alpha)}$ in all terms drops out, and finally we divide by $a(x)^{\alpha /(1-\alpha)}$ to obtain the result.
} 
which is stated in Proposition (8). We call the solution of this equation $x^{o}$. The existence and uniqueness of $x^{o}$ follow from Proposition 2 because the right-hand side is monotonically increasing in $x$, zero in the limit $x \rightarrow-\infty$ and $\infty$ in the limit $x \rightarrow \infty$. Because the right-hand side is increasing in $x$, it follows that (A.15) holds for all $x>x^{o}$. This means the investor should not invest for any $x>x^{o}$. Indeed, the optimal decision rule is to invest when $x \leq x^{*}$, where $x^{*} \leq x^{o}$ with equality only in the case $\sigma^{2}=0$. Hence we should not invest when $x \geq x^{o}$. The next section in this appendix shows that we should invest as soon as $x \leq x^{*}$.

3. Comparative statics: $\alpha$ does not affect $a(x)$ while it increases the right hand side; since the right hand side is increasing in $x$, this implies that the solution for $x$ must be decreasing in $\alpha$.

\section{A.13 Solution for Cobb-Douglas case}

This section solves the ODE (A.14) satisfied by the unknown function $v(x)$. To solve the ODE, it is useful to introduce the coordinate transformation $x \mapsto z(x)$, where $z:=x-\frac{\alpha \sigma^{2}}{1-\alpha}$. Furthermore, let $w(\cdot)=v\left(\cdot+\frac{\alpha \sigma^{2}}{1-\alpha}\right)$, such that $w(z)=v(x)$. Because $z$ is equal to $x$ shifted by a constant, it follows that $v^{\prime}(x)=w^{\prime}(z)$ and $v^{\prime \prime}(x)=w^{\prime \prime}(z)$. Hence equation (A.14) can equivalently written as

$$
0=\frac{\sigma^{2}}{2} w^{\prime \prime}(z)-z w^{\prime}(z)+\nu w(z), \quad \forall z \in \mathbb{R},
$$

which is recognized as Hermite's ODE. If $\nu$ were a positive integer (which it is not), then a possible solution would be $\mathrm{H}_{\nu}(z / \sigma)$, where $\mathrm{H}_{\nu}(\cdot)$ is a Hermite polynomial of degree $\nu$. However, we shall see that the desired solution can still be written as $\mathrm{H}_{\nu}(z / \sigma)$, as long as the Hermite polynomial is interpreted in a generalized sense, where $\nu$ is allowed to be non-integer and negative.

To show that this is the case, we propose a series expansion $w(z)=\sum_{i=0}^{\infty} c_{i} z^{i}$ as our candidate solution. For $w^{\prime \prime}(z)$, we have

$$
\frac{\sigma^{2}}{2} w^{\prime \prime}(z)=\frac{\sigma^{2}}{2} \frac{\mathrm{d}^{2}}{\mathrm{~d} z^{2}} \sum_{i=0}^{\infty} c_{i} z^{i}=\sigma^{2} c_{2}+\frac{\sigma^{2}}{2} \sum_{i=1}^{\infty} c_{i+2}(i+2)(i+1) z^{i}
$$

while for $w^{\prime}(z)$ it holds that

$$
-z w^{\prime}(z)=-z \frac{\mathrm{d}}{\mathrm{d} z} \sum_{i=0}^{\infty} c_{i} z^{i}=-\sum_{i=1}^{\infty} i c_{i} z^{i}
$$

Substituting these equalities into Hermite's ODE (A.18) gives

$$
\begin{aligned}
0 & =\sigma^{2} c_{2}+\nu c_{0}+\sum_{i=1}^{\infty}\left[\frac{\sigma^{2}}{2} c_{i+2}(i+2)(i+1)-(i-\nu) c_{i}\right] z^{i} \\
& =\sum_{i=0}^{\infty}\left[\frac{\sigma^{2}}{2} c_{i+2}(i+2)(i+1)-(i-\nu) c_{i}\right] z^{i}, \quad \forall z \in \mathbb{R} .
\end{aligned}
$$

This equation holds only if coefficient in square brackets is zero for every single value of $i=0,1,2,3, \cdots$. Hence we need

$$
c_{i+2}=\frac{2}{\sigma^{2}} \frac{i-\nu}{(i+2)(i+1)} c_{i}, \quad \forall i=0,1,2,3, \cdots
$$

This recursive equation relates $c_{i+2}$ to $c_{i}$. Two independent solutions $w_{k}(z)$ for $k=1,2$ may be obtained by starting with an arbitrary value of $c_{0}$ (or $c_{1}$ ) and considering only even (or odd) powers as follows:

$$
\begin{aligned}
& w_{1}(z)=c_{0}\left[1+\frac{2}{\sigma^{2}} \frac{0-\nu}{2 \times 1} z^{2}+\frac{2^{2}}{\sigma^{4}} \frac{0-\nu}{2 \times 1} \frac{2-\nu}{4 \times 3} z^{4}+\cdots\right]=: c_{0}{ }_{1} F_{1}\left(\frac{-\nu}{2}, \frac{1}{2}, \frac{z^{2}}{\sigma^{2}}\right), \\
& w_{2}(z)=c_{1}\left[z+\frac{2}{\sigma^{2}} \frac{1-\nu}{3 \times 2} z^{3}+\frac{2^{2}}{\sigma^{4}} \frac{1-\nu}{3 \times 2} \frac{3-\nu}{5 \times 4} z^{5}+\cdots\right]=: \frac{c_{1} z}{\sigma}{ }_{1} F_{1}\left(\frac{-(\nu-1)}{2}, \frac{3}{2}, \frac{z^{2}}{\sigma^{2}}\right),
\end{aligned}
$$

where, on the far right-hand side, we use the definition of the confluent hypergeometric function of the first kind, denoted by ${ }_{1} F_{1}(\cdot, \cdot, \cdot)$, see e.g. Abramovich and Stegun (1972), p. 504, their equation (13.1.2). In the limit where $z \rightarrow \infty$, these functions behave like

$$
\begin{aligned}
& w_{1}(z)=\quad c_{0}{ }_{1} F_{1}\left(\frac{-\nu}{2}, \frac{1}{2}, \frac{z^{2}}{\sigma^{2}}\right) \quad \approx c_{0} \frac{\sqrt{\pi}}{\Gamma\left(\frac{-\nu}{2}\right)}\left(\frac{z}{\sigma}\right)^{-(1+\nu)} \mathrm{e}^{z^{2} / \sigma^{2}}, \quad \text { as } z \rightarrow \infty \\
& w_{2}(z)=c_{1} z_{1} F_{1}\left(\frac{-(\nu-1)}{2}, \frac{3}{2}, \frac{z^{2}}{\sigma^{2}}\right) \approx c_{1} \frac{\sqrt{\pi}}{2 \Gamma\left(\frac{1-\nu}{2}\right)}\left(\frac{z}{\sigma}\right)^{-(1+\nu)} \mathrm{e}^{z^{2} / \sigma^{2}}, \quad \text { as } z \rightarrow \infty
\end{aligned}
$$

see e.g. Abramovich and Stegun (1972), p. 504, their equation (13.1.4). By the approximation sign " $\approx$ ", we mean that the 
ratio of the quantities on its left and right goes to unity as $z \rightarrow \infty$. Next we recall Proposition 1, which says that the steadystate distribution of $x$ is normal with a variance of $\sigma^{2} / 2$. It follows that the steady-state distribution of $z=x-\alpha \sigma^{2} /(1-\alpha)$ is also normal with a variance equal to $\sigma^{2} / 2$. This implies that the steady-state probability density of $z$ decays proportional to $\exp \left(-z^{2} \sigma^{2}\right)$ in the limit where $z \rightarrow \infty$. Hence, if we multiply the steady-state density by $w_{1}(z)$ or $w_{2}(z)$, then as $z \rightarrow \infty$ the product looks like $(z / \sigma)^{-(1+\nu)}$, which is not an integrable function (recall that $\nu<0$ ). Hence, for $c_{0}, c_{1} \geq 0$, we have

$$
\mathrm{E}\left[w_{1}(z) \mid z>0\right]=\mathrm{E}\left[w_{2}(z) \mid z>0\right]=\infty .
$$

In such circumstances, Dixit and Pindyck (1994) (pp. 181-2) use a 'no-bubble argument' to rule out a solution with undesirable asymptotic properties. In our case, however, this rules out both our candidate solutions. Hence we must pick $c_{0}$ and $c_{1}$ so that the linear combination $w(z)=w_{1}(z)+w_{2}(z)$ grows much slower than $(x / \sigma)^{-(1+\nu)} \exp \left(z^{2} \sigma^{2}\right)$ in the limit where $z \rightarrow \infty$. From (A.19), it follows that the following choice is adequate:

$$
c_{0}=C 2^{\nu} \frac{\sqrt{\pi}}{\Gamma\left(\frac{1-\nu}{2}\right)}, \quad c_{1}=-C 2^{\nu} \frac{2 \sqrt{\pi}}{\Gamma\left(\frac{-\nu}{2}\right)}
$$

where the factors of 2 are introduced for convenience and $C$ is an arbitrary constant. While it is clear that $c_{0}$ and $c_{1}$ must be of opposite sign for the desired cancellation to happen, our choice $c_{0}>0$ and $c_{1}<0$ is arbitrary (but convenient). When this choice is made, the full solution reads

$$
\begin{aligned}
w(z) & =w_{1}(z)+w_{2}(z), \\
& =C 2^{\nu} \sqrt{\pi}\left[\frac{1}{\Gamma\left(\frac{1-\nu}{2}\right)}{ }_{1} \mathrm{~F}_{1}\left(\frac{-\nu}{2}, \frac{1}{2}, \frac{z^{2}}{\sigma^{2}}\right)-\frac{2 z / \sigma}{\Gamma\left(\frac{-\nu}{2}\right)}{ }_{1} \mathrm{~F}_{1}\left(\frac{-(\nu-1)}{2}, \frac{3}{2}, \frac{z^{2}}{\sigma^{2}}\right)\right], \\
& =C \mathrm{H}_{\nu}\left(\frac{z}{\sigma}\right)
\end{aligned}
$$

where the third equality holds only if the Hermite polynomial is understood in a generalized sense, in which case it is defined as in the second line. Some computer packages, such as Wolfram's Mathematica, automatically compute $\mathrm{H}_{\nu}(\cdot)$ for negative non-integer values of $\nu$ by using the second line in (A.20) as the definition of the third. ${ }^{28}$. Other software packages, notably Matlab, return an error message, in which case the second rather than the third equality in (A.20) must be used.

Collecting our results so far and transforming back into our original variable $x$, the non-negative function $V(x, y)$ that behaves appropriately as $x \rightarrow \infty$ reads

$$
V(x, y)=C y^{1 /(1-\alpha)} \mathrm{e}^{x /(1-\alpha)} \mathrm{H}_{\nu}\left(\frac{x}{\sigma}-\frac{\alpha \sigma}{1-\alpha}\right)
$$

where $\nu=\frac{\mu}{1-\alpha}+\frac{\sigma^{2}}{2(1-\alpha)^{2}}-\rho<0$. This function is appropriately behaved at $x \rightarrow \infty$ because $\mathrm{H}_{\nu}(x) \approx(2 x)^{\nu}$ for $x \rightarrow \infty$. Finally, only two unknowns remain: (i) $C$ and (ii) $x^{*}$. These can be found by using a numerical procedure based on two remaining conditions (value matching and smooth pasting) as explained in part one of Appendix A.12. A simple implementation in Wolfram's Mathematica based on the function FindRoot is available on request.

\section{A.14 Proof of Proposition 9}

The Bellman equation for $V_{\lambda}(x, y)$ in (5.10) in conjunction with the definition of $W_{\lambda}(x, y)$ in $(5.10)$ implies that

$$
\begin{aligned}
(\rho-\mathcal{L}) W_{\lambda}(x, y) & =(\rho-\mathcal{L}) V_{\lambda}(x, y)-(\rho-\mathcal{L})\left[(1-\alpha) B^{*}(x, y)-\rho^{1 /(\alpha-1)}\right] \\
& =\lambda W_{\lambda}^{-}(x, y)+d(x, y)
\end{aligned}
$$

where $d(x, y)$ was defined in (5.12). Adding to both sides $\lambda W_{\lambda}(x, y)$ and using that $W_{\lambda}^{-}(x, y)+W_{\lambda}(x, y)=W_{\lambda}^{+}(x, y)$, we obtain (5.11). The other statements in Proposition 9 follow by the same arguments as in Propositions 6 and 7 .

\footnotetext{
${ }^{28}$ See http://functions.wolfram.com/05.01.26.0002.01.
} 


\section{A.15 Proof of equation (5.14)}

The equation $d(x, y)=0$ may be solved for $y$ to give

$$
\begin{aligned}
d(x, y)=0 \Rightarrow y & =\frac{\rho^{\alpha}}{\phi a(x)^{\alpha} \widetilde{\alpha}^{1-\alpha}\left[1-\rho \alpha a(x)-\frac{\sigma^{2}}{2} \frac{\alpha}{1-\alpha} \frac{a^{\prime}(x)^{2}}{a(x)}\right]^{1-\alpha}}, \\
& =\frac{\rho^{\alpha}(1-\alpha)^{1-\alpha}}{a(x)^{\alpha}\left[1-\rho \alpha a(x)-\frac{\sigma^{2}}{2} \frac{\alpha}{1-\alpha} \frac{a^{\prime}(x)^{2}}{a(x)}\right]^{1-\alpha}},
\end{aligned}
$$

where we have used $\phi:=\alpha^{-\alpha}(1-\alpha)^{\alpha-1}$ and $\widetilde{\alpha}:=\alpha^{\alpha /(1-\alpha)}$, such that $\phi \tilde{A} \widetilde{\alpha}^{1-\alpha}=(1-\alpha)^{\alpha-1}$. The optimal stopping boundary $y(x)$ must lie above the curve $d(x, y)=0$, such that on the domain $x \in\left(-\infty, x^{o}\right)$, we have

$$
\begin{aligned}
y(x) & \geq \frac{\rho^{\alpha}(1-\alpha)^{1-\alpha}}{a(x)^{\alpha}\left[1-\rho \alpha a(x)-\frac{\sigma^{2}}{2} \frac{\alpha}{1-\alpha} \frac{a^{\prime}(x)^{2}}{a(x)}\right]^{1-\alpha}}, \\
& \geq \frac{\rho^{\alpha}(1-\alpha)^{1-\alpha}}{a(x)^{\alpha}[1-\rho \alpha a(x)]^{1-\alpha}} \geq 1 .
\end{aligned}
$$

The last inequality follows because the function $\rho^{\alpha}(1-\alpha)^{1-\alpha} a(x)^{-\alpha}[1-\rho \alpha a(x)]^{\alpha-1}$ has a global minimum at $a(x)=\rho^{-1}$, where the value of the function is 1 . 